\title{
Remarks on $\tau$-functions for the difference Painlevé equations of type $E_{8}$
}

\author{
Masatoshi NOUMI*
}

\begin{abstract}
We investigate the structure of $\tau$-functions for the elliptic difference Painlevé equation of type $E_{8}$. Introducing the notion of ORG $\tau$-functions for the $E_{8}$ lattice, we construct some particular solutions which are expressed in terms of elliptic hypergeometric integrals. Also, we discuss how this construction is related to the framework of lattice $\tau$-functions associated with the configuration of generic nine points in the projective plane.
\end{abstract}

\section{Introduction}

There are several approaches to difference (or discrete) Painlevé equations associated with the root system of type $E_{8}$. In the geometric approach of Sakai [15], there are three discrete Painlevé equations with affine Weyl group symmetry of type $E_{8}^{(1)}$, which may be called rational, trigonometric and elliptic. They are formulated in the language of certain rational surfaces obtained from $\mathbb{P}^{2}$ by blowing-up at generic nine points, and regarded as master families of second order discrete Painlevé equations. On the other hand, Ohta-Ramani-Grammaticos [11] introduced the elliptic (difference) Painlevé equation of type $E_{8}$ and its $\tau$-functions as a discrete system on the root lattice of type $E_{8}$. Equivalence of these two approaches has been clarified by Kajiwara et al. [4, 5] in the framework of the birational affine Weyl group action on the configuration space of generic nine points in $\mathbb{P}^{2}$ and the associated lattice $\tau$-functions. Besides these approaches, the elliptic Painlevé equation is interpreted as the compatibility condition of certain linear difference equations in two ways by Rains [14 and by Noumi-Tsujimoto-Yamada [10]. Also, it is known that the elliptic difference Painlevé equation has particular solutions which are expressible in terms of elliptic hypergeometric functions as in Kajiwara et al. [4], Rains [12, 14] and Noumi-Tsujimoto-Yamada [10].

In this paper we introduce the notion of $O R G \tau$-functions, which is a reformulation of $\tau$-functions associated with the $E_{8}$ lattice proposed by Ohta-Ramani-Grammaticos [11. We fix a realization of the root lattice $P=Q\left(E_{8}\right)$ of type $E_{8}$ in the 8-dimensional

${ }^{*}$ Department of Mathematics, Kobe University, Rokko, Kobe 657-8501, Japan 2010 Mathematics Subject Classification. 39A20; 33E17, 33D70

Key words and Phrases. elliptic Painlevé equation, $E_{8}$ lattice, $\tau$-function, Hirota equation, Casorati determinant, elliptic hypergeometric integral 
complex vector space $V=\mathbb{C}^{8}$ endowed with the canonical symmetric bilinear form $(\cdot \mid \cdot): V \times V \rightarrow \mathbb{C}$ (Section 1), and take a subset $D \subseteq V$ such that $D+P \delta=D$, where $\delta \in \mathbb{C}^{*}$ is a nonzero constant. A function $\tau(x)$ defined on $D$ is called an ORG $\tau$-function if it satisfies a system of non-autonomous Hirota equations

$$
[(b \pm c \mid x)] \tau(x \pm a \delta)+[(c \pm a \mid x)] \tau(x \pm b \delta)+[(a \pm b \mid x)] \tau(x \pm c \delta)=0
$$

for all $C_{3}$-frames $\{ \pm a, \pm b, \pm c\}$ relative to $P$ (Section 2). Here $[z]$ denotes any nonzero entire function in $z \in \mathbb{C}$ which satisfies the three term relation (2.1), and each double sign in (0.1) indicates the product of two functions with different signs. When the domain $D$ is a disjoint union

$$
D_{c}=\bigsqcup_{n \in \mathbb{Z}} H_{c+n \delta}, \quad H_{c+n \delta}=\{x \in V \mid(\phi \mid x)=c+n \delta\},
$$

of parallel hyperplanes perpendicular to $\phi=\left(\frac{1}{2}, \frac{1}{2}, \ldots, \frac{1}{2}\right)$, each ORG $\tau$-function $\tau=\tau(x)$ on $D_{c}$ (of type $E_{8}$ ) is regarded as an infinite chain of ORG $\tau$-functions $\tau^{(n)}=\left.\tau\right|_{H_{c+n \delta}}$ on $H_{c+n \delta}$ of type $E_{7}(n \in \mathbb{Z})$ (Section 3 ). We say that a meromorphic ORG $\tau$-function $\tau(x)$ on $D_{c}$ is a hypergeometric $\tau$-function if $\tau^{(n)}(x)=0(n<0)$ and $\tau^{(0)}(x) \not \equiv 0$. Supposing that $\omega \in \Omega$ is a period of $[z]$, consider the case where $D=D_{\omega}$. In such a case, on the basis of a recursion theorem (Theorem 3.3) one can show that, if a given pair of functions $\tau^{(0)}(x)\left(x \in H_{\omega}\right)$ and $\tau^{(1)}(x)\left(x \in H_{\omega+\delta}\right)$ satisfies certain initial conditions, then there exists a unique hypergeometric $\tau$-function $\tau(x)\left(x \in D_{\omega}\right)$ having those $\tau^{(0)}(x)$, $\tau^{(1)}(x)$ as the first two components (Theorem 4.2). Furthermore, if one can specify a gauge factor for $\tau^{(1)}$ with respect to a $C_{3}$-frame of type $\mathrm{II}_{1}$, then for each $n=0,1,2, \ldots$ the $n$th component $\tau^{(n)}(x)\left(x \in H_{\omega+n \delta}\right)$ of the hypergeometric $\tau$-function is expressed in terms of a 2-directional Casorati determinant with respect to the $C_{3}$-frame of type $\mathrm{II}_{1}$ (Theorem 4.3). A proof of the recursion theorem (Thoerem 3.3) will be given in Appendix A.

In the latter half of this paper, we apply our arguments to the elliptic case for constructing hypergeometric ORG $\tau$-functions which are expressible in terms of elliptic hypergeometric integrals of Spiridonov [16, 17] and Rains [12, 13. The fundamental elliptic hypergeometric integral is the meromorphic function $I(u ; p, q)$ in eight variables $u=\left(u_{0}, u_{1}, \ldots, u_{7}\right) \in\left(\mathbb{C}^{*}\right)^{8}$ defined by

$$
I(u ; p, q)=\frac{(p ; p)_{\infty}(q ; q)_{\infty}}{4 \pi \sqrt{-1}} \int_{C} \frac{\prod_{k=0}^{7} \Gamma\left(u_{k} z^{ \pm 1} ; p, q\right)}{\Gamma\left(z^{ \pm 2} ; p, q\right)} \frac{d z}{z} .
$$

After recalling basic facts concerning elliptic hypergeometric integrals in Section 5, we present in Section 6 two types of explicit representations for the $W\left(E_{7}\right)$-invariant hypergeometric ORG $\tau$-functions, one by determinants (Theorem 6.1) and the other by multiple integrals (Theorem 6.2). Theorems 6.1 and 6.2 will be proved in Section 7 In particular we show there how the 2-directional Casorati determinant gives rise to the multiple elliptic hypergeometric integral of Rains [13]:

$$
\begin{aligned}
& I_{n}(u ; p, q) \\
& =\frac{(p ; p)_{\infty}^{n}(q ; q)_{\infty}^{n}}{2^{n} n !(2 \pi \sqrt{-1})^{n}} \int_{C^{n}} \prod_{i=1}^{n} \frac{\prod_{k=0}^{7} \Gamma\left(u_{k} z_{i}^{ \pm 1} ; p, q\right)}{\Gamma\left(z_{i}^{ \pm 2} ; p, q\right)} \prod_{1 \leq i<j \leq n} \theta\left(z_{i}^{ \pm 1} z_{j}^{ \pm 1} ; p\right) \frac{d z_{1} \cdots d z_{n}}{z_{1} \cdots z_{n}} .
\end{aligned}
$$


We also give some remarks in Section 8 on variations of hypergeometric ORG $\tau$-functions obtained by transformations in Theorem 2.3 .

In the final section, we discuss how the notion of ORG $\tau$-functions is related to that of lattice $\tau$-functions as discussed by Kajiwara et al. [5] in the context of the configuration space of generic nine points in $\mathbb{P}^{2}$. Some remarks are also given on the similar picture in the case of the configuration space of generic eight points in $\mathbb{P}^{1} \times \mathbb{P}^{1}$ as in KajiwaraNoumi-Yamada [6].

On this occasion I would like to give some personal comments on the position of this paper. The contents of this paper are not completely new, and many things presented here may be found in the literature. In fact, in the group of coauthors of [5], basic structures of the ORG $\tau$-functions were already known around the end of 2004, including the 2-directional Casorati determinant representation of the hypergeometric $\tau$-functions. (Some part of our discussion is reflected in the work on Masuda [8].) It was almost at the same time that Rains [12] clarified that his multiple elliptic hypergeometric integrals (0.4) satisfy certain quadratic relations of Hirota type that should be understood in the context of the elliptic Painlevé equation. It took a couple of years, however, for us to be able to confirm that the 2-directional Casorati determinants for a particular choice of $C_{3}$-frame of type $\mathrm{II}_{1}$ certainly give rise to the multiple elliptic hypergeometric integrals of Rains. As to further delay in presenting the detail of such an argument, I apologize just adding that it requires much more time and effort than might be imagined to accomplish a satisfactory paper in the language of a cultural sphere to which its author does not belong.

\section{Contents}

$1 \quad E_{8}$ lattice and $C_{l}$-frames $\underline{4}$

2 ORG $\tau$-functions $\quad \underline{7}$

$3 \quad E_{8} \tau$-function as an infinite chain of $E_{7} \tau$-functions $\quad \underline{10}$

4 Hypergeometric ORG $\tau$-functions $\quad \underline{14}$

5 Elliptic hypergeometric integrals $\underline{18}$

$6 W\left(E_{7}\right)$-invariant hypergeometric $\tau$-function $\underline{22}$

7 Proof of Theorems 6.2 and 6.3

8 Transformation of hypergeometric $\tau$-functions $\quad \underline{33}$

9 Relation to the framework of point configurations $\quad \underline{36}$

A Proof of Theorem $\underline{3.3} \quad \underline{46}$ 


\section{$1 \quad E_{8}$ lattice and $C_{l}$-frames}

We begin by recalling some basic facts concerning the root lattice of type $E_{8}$.

Let $V=\mathbb{C}^{8}=\mathbb{C} v_{0} \oplus \mathbb{C} v_{1} \oplus \cdots \oplus \mathbb{C} v_{7}$ be the 8-dimensional complex vector space with canonical basis $\left\{v_{0}, v_{1}, \ldots, v_{7}\right\}$, and $(\cdot \mid \cdot): V \times V \rightarrow \mathbb{C}$ the scalar product (symmetric bilinear form) such that $\left(v_{i} \mid v_{j}\right)=\delta_{i j}(i, j \in\{0,1, \ldots, 7\})$. Setting

$$
\phi=\left(\frac{1}{2}, \frac{1}{2}, \ldots, \frac{1}{2}\right)=\frac{1}{2}\left(v_{0}+v_{1}+\cdots+v_{7}\right) \in V,
$$

we realize the root lattice $Q\left(E_{8}\right)$ and the root system $\Delta\left(E_{8}\right)$ of type $E_{8}$ as

$$
P=\left\{a \in \mathbb{Z}^{8} \cup\left(\phi+\mathbb{Z}^{8}\right) \mid(\phi \mid a) \in \mathbb{Z}\right\} \subset V, \quad \Delta\left(E_{8}\right)=\{\alpha \in P \mid(\alpha \mid \alpha)=2\},
$$

respectively (see [1] for instance). This set $P$ forms a free $\mathbb{Z}$-module of rank 8 and the scalar product takes integer values on $P$. The theta series of the lattice $P=Q\left(E_{8}\right)$ is given by

$$
\sum_{a \in P} q^{(a \mid a)}=1+240 q^{2}+2160 q^{4}+6720 q^{6}+17520 q^{8}+30240 q^{10}+\cdots
$$

and $\Delta\left(E_{8}\right)$ consists of the following 240 vectors in $P$ :

$$
\begin{aligned}
& \text { (1) } \pm v_{i} \pm v_{j} \quad(0 \leq i<j \leq 7) \quad \cdots \quad\left(\begin{array}{l}
8 \\
2
\end{array}\right) \cdot 4=112 \text {, } \\
& \text { (2) } \quad \frac{1}{2}\left( \pm v_{0} \pm v_{1} \pm \cdots \pm v_{7}\right) \quad \text { (even number of }- \text { signs) } \quad \cdots \quad 2^{7}=128 \text {. }
\end{aligned}
$$

In this root system, we take the simple roots

$$
\alpha_{0}=\phi-v_{0}-v_{1}-v_{2}-v_{3}, \quad \alpha_{j}=v_{j}-v_{j+1} \quad(j=1, \ldots, 6), \quad \alpha_{7}=v_{7}+v_{0}
$$

corresponding to the Dynkin diagram

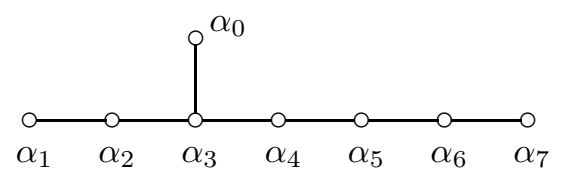

so that $P=Q\left(E_{8}\right)=\mathbb{Z} \alpha_{0} \oplus \mathbb{Z} \alpha_{1} \oplus \cdots \oplus \mathbb{Z} \alpha_{7}$. (For mutually distinct $i, j \in\{0,1, \ldots, 7\}$, $\left(\alpha_{i} \mid \alpha_{j}\right)=-1$ if the two nodes named $\alpha_{i}$ and $\alpha_{j}$ are connected by an edge, and $\left(\alpha_{i} \mid \alpha_{j}\right)=0$ otherwise.) The vector

$$
\phi=3 \alpha_{0}+2 \alpha_{1}+4 \alpha_{2}+6 \alpha_{3}+5 \alpha_{4}+4 \alpha_{5}+3 \alpha_{6}+2 \alpha_{7}
$$

is called the highest root with respect to the simple roots $\alpha_{0}, \alpha_{1}, \ldots, \alpha_{7}$. Note also that the weight lattice $P\left(E_{8}\right)$ coincides with the root lattice $Q\left(E_{8}\right)$ in this $E_{8}$ case.

For each $\alpha \in V$ with $(\alpha \mid \alpha) \neq 0$, we define the reflection $r_{\alpha}: V \rightarrow V$ with respect to $\alpha$ by

$$
r_{\alpha}(v)=v-\left(\alpha^{\vee} \mid v\right) \alpha \quad(v \in V)
$$


where $\alpha^{\vee}=2 \alpha /(\alpha \mid \alpha)$. The Weyl group $W\left(E_{8}\right)=\left\langle r_{\alpha}\left(\alpha \in \Delta\left(E_{8}\right)\right)\right\rangle$ of type $E_{8}$ acts on $V$ as a group of isometries; it stabilizes the root lattice $P=Q\left(E_{8}\right)$ and the root system $\Delta\left(E_{8}\right)$. We denote by $s_{j}=r_{\alpha_{j}}(j=0,1, \ldots, 7)$ the simple reflections. Then, $W\left(E_{8}\right)=$ $\left\langle s_{0}, s_{1}, \ldots, s_{7}\right\rangle$ is the Coxeter group associated with the Dynkin diagram (1.6). This group is generated by $s_{0}, s_{1}, \ldots, s_{7}$ with fundamental relations $s_{j}^{2}=1(j=0,1, \ldots, 7)$, $s_{i} s_{j}=s_{j} s_{i}$ for distinct $i, j \in\{0,1, \ldots, 7\}$ with $\left(\alpha_{i} \mid \alpha_{j}\right)=0$ and $s_{i} s_{j} s_{i}=s_{j} s_{i} s_{j}$ for distinct $i, j \in\{0,1, \ldots, 7\}$ with $\left(\alpha_{i} \mid \alpha_{j}\right)=-1$.

In the $E_{8}$ lattice $P=Q\left(E_{8}\right)$, the root lattice $Q\left(E_{7}\right)$ and the root system $\Delta\left(E_{7}\right)$ of type $E_{7}$ are realized as

$$
\begin{aligned}
& Q\left(E_{7}\right)=\{a \in P \mid(\phi \mid a)=0\}=\mathbb{Z} \alpha_{0} \oplus \mathbb{Z} \alpha_{1} \oplus \cdots \oplus \mathbb{Z} \alpha_{6}, \\
& \Delta\left(E_{7}\right)=\left\{\alpha \in \Delta\left(E_{8}\right) \mid(\phi \mid \alpha)=0\right\}
\end{aligned}
$$

respectively. The root system $\Delta\left(E_{7}\right)$ consists of the following 126 vectors:

$$
\begin{aligned}
& \text { (1) } \pm\left(v_{i}-v_{j}\right) \quad(0 \leq i<j \leq 7) \quad \cdots \quad\left(\begin{array}{l}
8 \\
2
\end{array}\right) \cdot 2=56 \text {, } \\
& \text { (2) } \quad \frac{1}{2}\left( \pm v_{0} \pm v_{1} \pm \cdots \pm v_{7}\right) \quad \text { (four - signs) } \quad \cdots \quad\left(\begin{array}{l}
8 \\
4
\end{array}\right)=70 .
\end{aligned}
$$

The highest root of $\Delta\left(E_{7}\right)$ with respect to the simple roots $\alpha_{0}, \alpha_{1}, \ldots, \alpha_{6}$ is given by

$$
v_{1}-v_{0}=2 \alpha_{0}+2 \alpha_{1}+3 \alpha_{2}+4 \alpha_{3}+3 \alpha_{4}+2 \alpha_{5}+\alpha_{6} .
$$

We denote by $W\left(E_{7}\right)=\left\langle r_{\alpha}\left(\alpha \in \Delta\left(E_{7}\right)\right)\right\rangle=\left\langle s_{0}, s_{1}, \ldots, s_{6}\right\rangle$ the Weyl group of type $E_{7}$. Note that $W\left(E_{7}\right)$ contains the symmetric group $\mathfrak{S}_{8}=\left\langle r_{v_{0}-v_{1}}, s_{1}, \ldots, s_{6}\right\rangle$ acting on $V$ through the permutation of $v_{0}, v_{1}, \ldots, v_{7}$, and is generated by this $\mathfrak{S}_{8}$ together with the reflection $s_{0}$ with respect to $\alpha_{0}=\phi-v_{0}-v_{1}-v_{2}-v_{3}$.

In this paper, the following notion of $C_{l}$-frames plays a fundamental role.

Definition 1.1 For $l=1,2, \ldots, 8$, a set $A=\left\{ \pm a_{0}, \pm a_{1}, \ldots, \pm a_{l-1}\right\}$ of $2 l$ vectors in $V$ is called a $C_{l}$-frame (relative to $P$ ), if the following two conditions are satisfied:

(1) $\quad\left(a_{i} \mid a_{j}\right)=\delta_{i j} \quad(0 \leq i, j<l)$,

(2) $\quad a_{i} \pm a_{j} \in P \quad(0 \leq i<j<l), \quad 2 a_{i} \in P \quad(0 \leq i<l)$.

This condition for $A=\left\{ \pm a_{0}, \ldots, \pm a_{l-1}\right\}$ means that the set of $2 l^{2}$ vectors

$$
\left.\Delta_{A}\left(C_{l}\right)=\left\{ \pm a_{i} \pm a_{j} \mid 0 \leq i<j<l\right)\right\} \cup\left\{ \pm 2 a_{i} \mid 0 \leq i<l\right\} \subset P
$$

form a root system of type $C_{l}$. For $l=1,2, \ldots, 8$, we denote by $\mathcal{C}_{l}$ the set of all $C_{l}$-frames. 


\section{Example 1.2 (Typical examples of $C_{8}$-frames)}

(0) $A_{0}=\left\{ \pm v_{0}, \pm v_{1}, \ldots, \pm v_{7}\right\}$.

(1) $A_{1}=\left\{ \pm a_{0}, \pm a_{1}, \ldots, \pm a_{7}\right\}$,

$$
a_{0}=\frac{1}{2}\left(v_{0}+v_{1}+v_{2}+v_{3}\right), \quad a_{4}=\frac{1}{2}\left(v_{4}-v_{5}-v_{6}+v_{7}\right),
$$$$
a_{1}=\frac{1}{2}\left(v_{0}+v_{1}-v_{2}-v_{3}\right), \quad a_{5}=\frac{1}{2}\left(-v_{4}+v_{5}-v_{6}+v_{7}\right),
$$$$
a_{2}=\frac{1}{2}\left(v_{0}-v_{1}+v_{2}-v_{3}\right), \quad a_{6}=\frac{1}{2}\left(-v_{4}-v_{5}+v_{6}+v_{7}\right),
$$$$
a_{3}=\frac{1}{2}\left(v_{0}-v_{1}-v_{2}+v_{3}\right), \quad a_{7}=\frac{1}{2}\left(v_{4}+v_{5}+v_{6}+v_{7}\right) \text {. }
$$

$$
\begin{aligned}
A_{2} & =\left\{ \pm a_{0}, \pm a_{1}, \ldots, \pm a_{7}\right\}, \\
a_{0} & =\frac{1}{2}\left(\phi+v_{0}-v_{7}\right), \quad a_{7}=\frac{1}{2}\left(\phi-v_{0}+v_{7}\right), \\
a_{j} & =v_{j}+\frac{1}{2}\left(v_{0}+v_{7}-\phi\right) \quad(j=1, \ldots, 6) .
\end{aligned}
$$

In the following, we denote by $N(v)=(v \mid v)$ the square norm of $v \in V$, and by $\varphi(v)=(\phi \mid v)$ the scalar product of $v$ with $\phi$. Also, for a subset $S \subseteq V$ given, we use the notations

$$
S_{N=k}=\{v \in S \mid(v \mid v)=k\}, \quad S_{\varphi=k}=\{v \in S \mid(\phi \mid v)=k\} \quad(k \in \mathbb{C})
$$

to refer to the level sets of $N$ and $\varphi$ respectively.

Note that each $C_{l}$-frame $(l=1,2, \ldots, 8)$ is formed by vectors in $\left(\frac{1}{2} P\right)_{N=1}=\frac{1}{2}\left(P_{N=4}\right)$. The set $P_{N=4}$ of all vectors in $P$ with square norm 4 consists of the following 2160 vectors that are classified into three groups under the action of the symmetric group $\mathfrak{S}_{8}$ :

$$
\begin{array}{lrr}
\text { (0) } \pm 2 v_{0} & \cdots & 8 \cdot 2=16, \\
\cdots & \cdots\left(\begin{array}{c}
8 \\
4
\end{array}\right) \cdot 2^{4}=1120, \\
(1) \pm v_{0} \pm v_{1} \pm v_{2} \pm v_{3} & \text { (odd number of - signs) } \cdots & 8 \cdot 2^{7}=1024 .
\end{array}
$$

The Weyl group $W\left(E_{8}\right)$ acts on $P_{N=4}$ transitively. In fact we have $P_{N=4} \cap P^{+}=$ $\left\{\phi-v_{0}+v_{1}\right\}$, where $P^{+}=\left\{v \in P \mid\left(\alpha_{j} \mid v\right) \geq 0(0 \leq j \leq 7)\right\}$ stands for the cone of dominant integral weights. It turns out that the stabilizer of $\phi-v_{0}+v_{1}$ is $W\left(D_{7}\right)$ and that

$$
P_{N=4} \stackrel{\sim}{\leftarrow} W\left(E_{8}\right) / W\left(D_{7}\right), \quad\left|P_{N=4}\right|=\left|W\left(E_{8}\right) / W\left(D_{7}\right)\right|=2160 .
$$

The following two propositions can be verified directly on the basis of this transitive action of $W\left(E_{8}\right)$ on $P_{N=4}$.

\section{Proposition 1.3}

(1) For each $a \in\left(\frac{1}{2} P\right)_{N=1}$, there exists a unique $C_{8}$-frame containing a.

(2) The set $\left(\frac{1}{2} P\right)_{N=1}$ is the disjoint union of all $C_{8}$-frames: $\left(\frac{1}{2} P\right)_{N=1}=\bigsqcup_{A \in \mathcal{C}_{8}} A$.

(3) The number of $C_{8}$-frames is given by $\left|\mathcal{C}_{8}\right|=2160 / 16=135$.

Proposition 1.4 Fix a positive integer $l \in\{1, \ldots, 8\}$.

(1) The Weyl group $W\left(E_{8}\right)$ acts transitively on the set $\mathcal{C}_{l}$ of all $C_{l}$-frames.

(2) Each $C_{l}$-frame is contained in a unique $C_{8}$-frame.

(3) The number of $C_{l}$-frames is given by $\left|\mathcal{C}_{l}\right|=135 \cdot\left(\begin{array}{l}8 \\ l\end{array}\right)$. 


\section{ORG $\tau$-functions}

In this section we introduce the notion of $O R G$-functions, which is a reformulation of $\tau$-functions associated with the $E_{8}$ lattice proposed by Ohta-Ramani-Grammaticos [11.

We fix once for all a nonzero entire function $[z]$ in $z \in \mathbb{C}$ satisfying the three-term relation

$$
[\beta \pm \gamma][z \pm \alpha]+[\gamma \pm \alpha][z \pm \beta]+[\alpha \pm \beta][z \pm \gamma]=0 \quad(z, \alpha, \beta, \gamma \in \mathbb{C})
$$

Throughout this paper, we use the abbreviation $[\alpha \pm \beta]=[\alpha+\beta][\alpha-\beta]$ with a double sign indicating the product of two factors. From (2.1) it follows that that $[z]$ is an odd function $([-z]=-[z],[0]=0)$. We remark that the three-term relation (2.1) can be written alternatively as

$$
[z \pm \alpha][w \pm \beta]-[z \pm \beta][w \pm \alpha]=[z \pm w][\alpha \pm \beta] \quad(z, w, \alpha, \beta \in \mathbb{C})
$$

or

$$
\frac{[z \pm \alpha]}{[z \pm \beta]}-\frac{[w \pm \alpha]}{[w \pm \beta]}=\frac{[z \pm w][\alpha \pm \beta]}{[z \pm \beta][w \pm \beta]} \quad(z, w, \alpha, \beta \in \mathbb{C}) .
$$

It is known that the functional equation (2.1) for $[z]$ implies that the set of zeros $\Omega=$ $\{\omega \in \mathbb{C} \mid[\omega]=0\}$ form a closed discrete subgroup of the additive group $\mathbb{C}$. Furthermore, such a function $[z]$ belongs to one of the following three classes, rational, trigonometric or elliptic, according to the rank of $\Omega([19])$ :

$$
\begin{array}{llll}
(0) & \text { rational } & :[z]=e\left(c_{0} z^{2}+c_{1}\right) z & (\Omega=0), \\
(1) \quad \text { trigonometric } & :[z]=e\left(c_{0} z^{2}+c_{1}\right) \sin \left(\pi z / \omega_{1}\right) & \left(\Omega=\mathbb{Z} \omega_{1}\right), \\
(2) \quad \text { elliptic } & :[z]=e\left(c_{0} z^{2}+c_{1}\right) \sigma(z \mid \Omega) & \left(\Omega=\mathbb{Z} \omega_{1} \oplus \mathbb{Z} \omega_{2}\right),
\end{array}
$$

where $e(z)=e^{2 \pi \sqrt{-1} z}$, and $c_{0}, c_{1} \in \mathbb{C}$. In the elliptic case, $\Omega$ is generated by complex numbers $\omega_{1}, \omega_{2}$ which are linearly independent over $\mathbb{R}$, and $\sigma(z \mid \Omega)$ stands for the Weierstrass sigma function associated with the period lattice $\Omega=\mathbb{Z} \omega_{1} \oplus \mathbb{Z} \omega_{2}$. In the trigonometric and elliptic cases, $[z]$ is quasi-periodic with respect to $\Omega$ in the following sense:

$$
[z+\omega]=\epsilon_{\omega} e\left(\eta_{\omega}\left(z+\frac{\omega}{2}\right)\right)[z] \quad(\omega \in \Omega)
$$

where $\eta_{\omega} \in \mathbb{C}(\omega \in \Omega)$ are constants such that $\eta_{\omega+\omega^{\prime}}=\eta_{\omega}+\eta_{\omega^{\prime}}\left(\omega, \omega^{\prime} \in \Omega\right)$, and $\epsilon_{\omega}=+1$ or -1 according as $\omega \in 2 \Omega$ or $\omega \notin 2 \Omega$.

In what follows we fix a nonzero constant $\delta \in \mathbb{C}$ such that $\mathbb{Z} \delta \cap \Omega=\{0\}$. Let $D$ be a subset of $V=\mathbb{C}^{8}$ stable under the translation by $P \delta$, namely $D+P \delta=D$.

Definition 2.1 A function $\tau(x)$ defined over $D$ is called an $O R G \tau$-function if it satisfies the non-autonomous Hirota equations

$$
\mathrm{H}(a, b, c): \quad[(b \pm c \mid x)] \tau(x \pm a \delta)+[(c \pm a \mid x)] \tau(x \pm b \delta)+[(a \pm b \mid x)] \tau(x \pm c \delta)=0
$$

for all $C_{3}$-frames $\{ \pm a, \pm b, \pm c\}$ relative to $P$. 


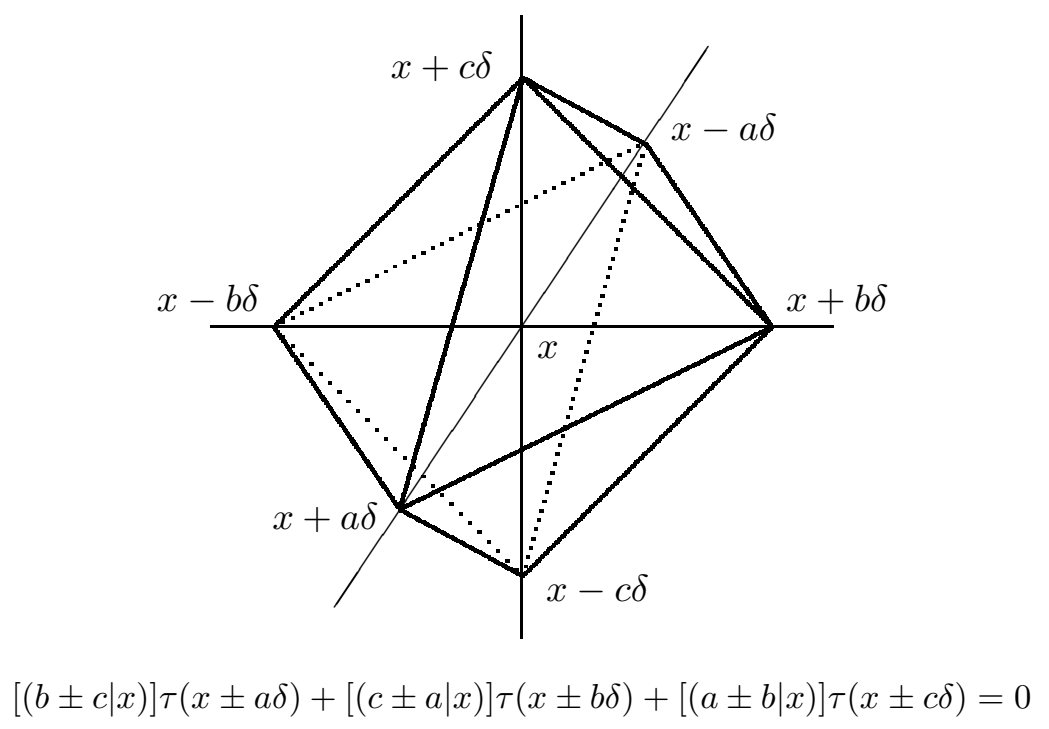

Figure 1: Non-autonomous Hirota equation

A $C_{3}$-frame $\{ \pm a, \pm b, \pm c\}$ defines an octahedron in $V$ of which the twelve edges and the three diagonals are vectors in the $E_{8}$ lattice $P$. Hence, if one of the six vertices $\{x \pm a \delta, x \pm b \delta, x \pm c \delta\}$ belongs to $D$, the other five belong to $D$ as well by the property of a $C_{3}$-frame. Also, by Proposition 1.4 the number of $C_{3}$-frames is $\left|\mathcal{C}_{3}\right|=135 \cdot\left(\begin{array}{l}8 \\ 3\end{array}\right)=7560$. Hence, the equation to be satisfied by an ORG $\tau$-function is a system of 7560 nonautonomous Hirota equations, which we call the $O R G$ system of type $E_{8}$. (A bilinear equation of the form (2.6) is also called a Hirota-Miwa equation.)

In Definition 2.1, as the independent variables of $\tau(x)$ one can take both discrete and continuous variables. The two extreme cases of the domain $D$ are:

$$
\text { (1) } D=v+P \delta \quad \text { (fully discrete), (2) } D=V \quad \text { (fully continuous). }
$$

There are intermediate cases where $D$ is a disjoint union of a countable family of affine subspaces. In such cases, we assume that $\tau(x)$ is a holomorphic (or meromorphic) function on $D$.

Proposition 2.2 For any constant $c \in \mathbb{C}$, the entire function

$$
\tau(x)=\left[\frac{1}{2 \delta}(x \mid x)+c\right] \quad(x \in V)
$$

is an $O R G \tau$-function on $V$.

Proof: Noting that $\tau(x \pm a \delta)=\left[\frac{1}{2 \delta}(x \mid x)+\frac{\delta}{2}+c \pm(a \mid x)\right]$, set

$$
z=\frac{1}{2 \delta}(x \mid x)+\frac{\delta}{2}+c, \quad \alpha=(a \mid x), \quad \beta=(b \mid x), \quad \gamma=(c \mid x) .
$$

Then the Hirota equation $\mathrm{H}(a, b, c)$ reduces to the functional equation (2.1).

The ORG $\tau$-function (2.8) can be regarded as the canonical solution of the ORG system. We give below some remarks on transformations of an ORG $\tau$-function. 
Theorem 2.3 Let $D$ be a subset of $V$ with $D+P \delta=D$, and $\tau(x)$ an $O R G \tau$-function on $D$.

(1) (Multiplication by an exponential function) For any constants $k, c \in \mathbb{C}$, any vector $v \in V$ and $\epsilon= \pm 1$, the function

$$
\widetilde{\tau}(x)=e(k(x \mid x)+(v \mid x)+c) \tau(\epsilon x) \quad(x \in \epsilon D)
$$

is an $O R G$-function on $\epsilon D$.

(2) (Transformation by $W\left(E_{8}\right)$ ) For any $w \in W\left(E_{8}\right)$, the function $w \cdot \tau$ defined by

$$
(w \cdot \tau)(x)=\tau\left(w^{-1} \cdot x\right) \quad(x \in w \cdot D)
$$

is an ORG $\tau$-function on $w . D$.

(3) (Translation by a period) For any period $\omega \in \Omega$ and any $v \in P$, the function $\widetilde{\tau}$ defined by

$$
\begin{aligned}
& \widetilde{\tau}(x)=e(S(x ; v, \omega)) \tau(x-v \omega) \quad(x \in D+v \omega), \\
& S(x ; v, \omega)=\frac{\eta_{\omega}}{2 \delta^{2}}(v \mid x)(x \mid x-v \omega),
\end{aligned}
$$

is an $O R G$-function on $D+v \omega$.

Proof: Since Statements (1) and (2) are straightforward, we give a proof of (3) only. Set $y=x-v \omega \in D$ so that

$$
[(b \pm c \mid x)] \widetilde{\tau}(x \pm a \delta)=[(b \pm c \mid y+v \omega)] e(S(x \pm a \delta ; v, \omega)) \tau(y \pm a \delta) .
$$

Since

$$
\begin{aligned}
{[(b+c \mid y+v \omega)] } & =[(b+c \mid y)+(b+c \mid v) \omega] \\
& =\epsilon_{(b+c \mid v) \omega} e\left(\eta_{\omega}(b+c \mid v)\left(b+c \mid y+v \frac{\omega}{2}\right)\right)[(b+c \mid y)]
\end{aligned}
$$

we have

$$
\begin{aligned}
& {[(b \pm c \mid y+v \omega)]} \\
& =\epsilon_{(b+c \mid v) \omega} \epsilon_{(b-c \mid v) \omega}[(b \pm c \mid y)] e\left(2 \eta_{\omega}\left((b \mid v) b+(c \mid v) c \mid y+v \frac{\omega}{2}\right)\right) \\
& =\epsilon_{(b+c \mid v) \omega} \epsilon_{(b-c \mid v) \omega}[(b \pm c \mid y)] e\left(2 \eta_{\omega}\left((b \mid v) b+(c \mid v) c \mid x-v \frac{\omega}{2}\right)\right) .
\end{aligned}
$$

On the other hand,

$$
S(x+a \delta ; v, w)+S(x-a \delta ; v, w)=\frac{\eta_{\omega}}{\delta^{2}}(v \mid x)\left((v \mid x-v \omega)+\delta^{2}\right)+2 \eta_{\omega}(a \mid v)\left(a \mid x-v \frac{\omega}{2}\right) .
$$

This implies

$$
\begin{aligned}
& {[(b \pm c \mid x)] \widetilde{\tau}(x \pm a \delta)} \\
& =\epsilon_{(b+c \mid v) \omega} \epsilon_{(b-c \mid v) \omega}[(b \pm c \mid y)] \tau(y \pm a \delta) \\
& \quad \cdot e\left(\frac{\eta_{\omega}}{\delta^{2}}(v \mid x)\left((v \mid x-v \omega)+\delta^{2}\right)\right) e\left(2 \eta_{\omega}\left((a \mid v) a+(b \mid v) b+(c \mid v) c \mid x-v \frac{\omega}{2}\right)\right) .
\end{aligned}
$$


Hence, validity of the Hirota equation for $\widetilde{\tau}(x)$ reduces to proving

$$
\epsilon_{(b+c \mid v) \omega} \epsilon_{(b-c \mid v) \omega}=\epsilon_{(c+a \mid v) \omega} \epsilon_{(c-a \mid v) \omega}=\epsilon_{(a+b \mid v) \omega} \epsilon_{(a-b \mid v) \omega} .
$$

Since this holds trivially for $\omega \in 2 \Omega$, we assume $\omega \notin 2 \Omega$. In view of the transitive action of $W\left(E_{8}\right)$ on $\mathcal{C}_{3}$, we may assume $\{ \pm a, \pm b, \pm c\}=\left\{ \pm v_{0}, \pm v_{1}, \pm v_{2}\right\}$. Then, for distinct $i, j \in\{0,1,2\}$, we have

$$
\begin{array}{ccc}
v \in \mathbb{Z}^{8} & \Longrightarrow & \left(v_{i}+v_{j} \mid v\right) \equiv\left(v_{i}-v_{j} \mid v\right) \bmod 2, \\
v \in \phi+\mathbb{Z}^{8} & \Longrightarrow & \left(v_{i}+v_{j} \mid v\right) \not \equiv\left(v_{i}-v_{j} \mid v\right) \bmod 2 .
\end{array}
$$

Since

$$
\epsilon_{k \omega} \epsilon_{l \omega}=\left\{\begin{array}{cl}
+1 & (k \equiv l \bmod 2) \\
-1 & (k \neq l \quad \bmod 2)
\end{array}\right.
$$

for $\omega \notin 2 \Omega, \epsilon_{\left(v_{i}+v_{j} \mid v\right) \omega} \epsilon_{\left(v_{i}-v_{j} \mid v\right) \omega}$ takes the value +1 or -1 according as $v \in P$ belongs to $\mathbb{Z}^{8}$ or $\phi+\mathbb{Z}^{8}$, regardless of the choice of the pair $i, j$. This completes the proof of (3).

We remark that the composition of two translations of (3) by $a \omega$ and by $b \omega$ for $a, b \in P$ and $\omega \in \Omega$ results essentially in the same transformation as the translation by $(a+b) \omega$. In fact we have

$$
\begin{aligned}
& e(S(x ; b \omega) e(S(x-b \omega ; a, \omega)) \tau(x-(a+b) \omega) \\
& =e(k(x \mid x)+(v \mid x)+c) S(x ; a+b, \omega) \tau(x-(a+b) \omega)
\end{aligned}
$$

for some $k, c \in \mathbb{C}$ and $v \in V$.

\section{$3 \quad E_{8} \tau$-function as an infinite chain of $E_{7} \tau$-functions}

Recall that the root lattice $Q\left(E_{7}\right)$ of type $E_{7}$ is the orthogonal complement of $\phi$ in $P=Q\left(E_{8}\right)$. In what follows, we denote by

$$
H_{\kappa}=\{x \in V \mid(\phi \mid x)=\kappa\} \quad(\kappa \in \mathbb{C})
$$

the hyperplanes in $V$ defined as the level sets of $\varphi=(\phi \mid \cdot)$. Fixing a constant $c \in \mathbb{C}$, we now consider the case where the domain $D$ of an ORG $\tau$-function is a disjoint union of parallel hyperplanes

$$
D_{c}=\bigsqcup_{n \in \mathbb{Z}} H_{c+n \delta} .
$$

Note that each component $H_{c+n \delta}(n \in \mathbb{Z})$ is invariant under the action of $W\left(E_{7}\right)$ and the translation by $Q\left(E_{7}\right) \delta$, and that the whole set $D$ is invariant under the translation by $Q\left(E_{8}\right) \delta$. In this situation, we regard a function $\tau(x)$ on $D_{c}$ as an infinite family of functions $\tau^{(n)}(x)$ on $H_{c+n \delta}(n \in \mathbb{Z})$ defined by restriction as $\tau^{(n)}=\left.\tau\right|_{H_{c+n \delta}}$ for $n \in \mathbb{Z}$. In order to investigate the system of Hirota equations for $\tau^{(n)}(x)(n \in \mathbb{Z})$, we classify them under the action of the Weyl group $W\left(E_{7}\right)$. 
For a $C_{l}$-frame $A=\left\{ \pm a_{0}, \ldots, \pm a_{l-1}\right\}$ given, we consider the multiset

$$
\varphi(A)=\left\{ \pm \varphi\left(a_{0}\right), \ldots, \pm \varphi\left(a_{l-1}\right)\right\},
$$

where $\varphi(v)=(\phi \mid v)(v \in V)$. As to the three $C_{8}$-frames of Example 1.2, we have

$$
\varphi\left(A_{0}\right)=\left\{\left( \pm \frac{1}{2}\right)^{8}\right\}, \quad \varphi\left(A_{1}\right)=\varphi\left(A_{2}\right)=\left\{( \pm 1)^{2}, 0^{12}\right\} .
$$

Here the symbol $c^{n}$ (resp. $\left.( \pm c)^{n}\right)$ indicates that $c$ appears (resp. both $+c$ and $-c$ appear) with multiplicity $n$ in the multiset. We say that a $C_{8}$-frame is of type I if $\varphi(A)=\left\{\left( \pm \frac{1}{2}\right)^{8}\right\}$, and of type II if $\varphi(A)=\left\{( \pm 1)^{2}, 0^{12}\right\}$.

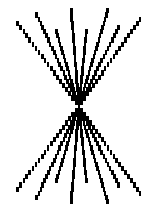

$C_{8}$-frame of type I

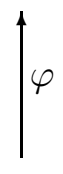

$$
C_{8} \text {-frame of type II }
$$

Proposition 3.1 Any $C_{8}$-frame is either of type I or of type II. Furthermore, these two types give the decomposition of the set $\mathcal{C}_{8}$ of all $C_{8}$-frames into $W\left(E_{7}\right)$-orbits:

$$
\begin{aligned}
& \mathcal{C}_{8}=\mathcal{C}_{8, \mathrm{I}} \sqcup \mathcal{C}_{8, \mathrm{II}}, \quad\left|\mathcal{C}_{8, \mathrm{I}}\right|=72, \quad\left|\mathcal{C}_{8, \mathrm{II}}\right|=63 ; \\
& \mathcal{C}_{8, \mathrm{I}}=W\left(E_{7}\right) A_{0}, \quad \mathcal{C}_{8, \mathrm{II}}=W\left(E_{7}\right) A_{1}=W\left(E_{7}\right) A_{2} .
\end{aligned}
$$

In order to analyze the $W\left(E_{7}\right)$-orbits in $\mathcal{C}_{8}$, we first decompose $P_{N=4}$ into $W\left(E_{7}\right)$-orbits. As a result, $P_{N=4}$ decomposes into the form

$$
P_{N=4}=P_{N=4, \varphi=2} \sqcup P_{N=4, \varphi=1} \sqcup P_{N=4, \varphi=0} \sqcup P_{N=4, \varphi=-1} \sqcup P_{N=4, \varphi=-2},
$$

and each level set of $\varphi$ forms a single $W\left(E_{7}\right)$-orbit. The five $W\left(E_{7}\right)$-orbits are described as follows.

\begin{tabular}{|c||c|c|c|c|c|}
\hline$\varphi$ & 2 & 1 & 0 & -1 & -2 \\
\hline \hline representative & $\phi-v_{0}+v_{1}$ & $\phi-2 v_{0}$ & $\phi-2 v_{0}-v_{6}-v_{7}$ & $-2 v_{0}$ & $-\phi-v_{0}+v_{1}$ \\
\hline stabilizer & $W\left(D_{6}\right)$ & $W\left(A_{6}\right)$ & $W\left(D_{5} \times A_{1}\right)$ & $W\left(A_{6}\right)$ & $W\left(D_{6}\right)$ \\
\hline cardinality & 126 & 576 & 756 & 576 & 126 \\
\hline
\end{tabular}

The "representative" indicates a unique vector $v$ in the orbit such that $\left(\alpha_{j} \mid v\right) \geq 0(j=$ $0,1, \ldots, 6)$. According to this decomposition of $P_{N=4},\left(\frac{1}{2} P\right)_{N=1}=\frac{1}{2}\left(P_{N=4}\right)$ decomposes into the five $W\left(E_{7}\right)$-orbits with $\varphi=1, \frac{1}{2}, 0,-\frac{1}{2},-1$. Proposition 3.1 follows from the fact that $\frac{1}{2} \phi-v_{0}$ and $v_{0}$ belong to $C_{8}$-frames of type I, and $\frac{1}{2}\left(\phi-v_{0}+v_{1}\right), \frac{1}{2}\left(\phi-v_{6}-v_{7}\right)-v_{0}$, and $\frac{1}{2}\left(-\phi-v_{0}+v_{1}\right)$ to those of type II. Note that, among the $63 C_{8}$-frames of type II, 35 are obtained from $A_{1}$, and 28 from $A_{2}$ of Example 1.2 by the action of the symmetric group $\mathfrak{S}_{8} \subset W\left(E_{7}\right)$. 


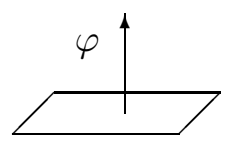

(I)

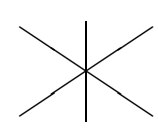

$\left(\mathrm{II}_{0}\right)$
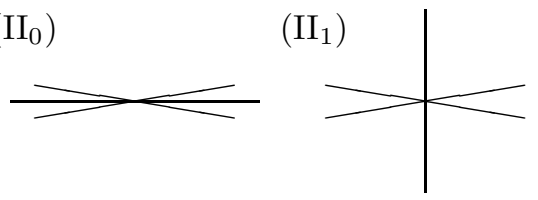

$56 \cdot 72=4032 \quad 20 \cdot 63=1260$
$30 \cdot 63=1890$

$\left(\mathrm{II}_{2}\right)$

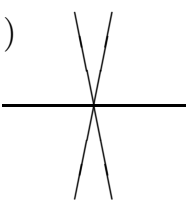

$6 \cdot 63=378$

Figure 2: Four types of $7560 C_{3}$-frames

We remark that in any $C_{8}$-frame $\left\{ \pm a_{0}, \pm a_{1}, \ldots, \pm a_{7}\right\}$ with

$$
\text { (I) : } \quad\left(\phi \mid a_{j}\right)=\frac{1}{2} \quad(j=0,1, \ldots, 7)
$$

we have $\frac{1}{2}\left(a_{0}+a_{1}+\cdots+a_{7}\right)=\phi$. Also, in any $C_{8}$-frame $\left\{ \pm a_{0}, \pm a_{1}, \ldots, \pm a_{7}\right\}$ with

$$
\text { (II) }: \quad\left(\phi \mid a_{0}\right)=\left(\phi \mid a_{7}\right)=1, \quad\left(\phi \mid a_{j}\right)=0 \quad(j=1, \ldots, 6),
$$

we have $a_{0}+a_{7}=\phi$. Since these statements are $W\left(E_{7}\right)$-invariant, by Proposition 3.1 we have only to check the cases of $A_{0}$ and $A_{1}$ of Example 1.2, respectively.

By Proposition 1.4, each $C_{3}$-frame is contained in a unique $C_{8}$-frame. Hence, by Proposition 3.1 we obtain the following classification of $C_{3}$-frames.

Proposition 3.2 The set $\mathcal{C}_{3}$ of all $C_{3}$-frames decomposes into four $W\left(E_{7}\right)$-orbits:

$$
\mathcal{C}_{3}=\mathcal{C}_{3, \mathrm{I}} \sqcup \mathcal{C}_{3, \mathrm{II}_{0}} \sqcup \mathcal{C}_{3, \mathrm{II}_{1}} \sqcup \mathcal{C}_{3, \mathrm{II}} .
$$

These four $W\left(E_{7}\right)$-orbits are characterized as follows.

\begin{tabular}{|c||c|c|c|c|}
\hline type & $\mathrm{I}$ & $\mathrm{II}_{0}$ & $\mathrm{II}_{1}$ & $\mathrm{II}_{2}$ \\
\hline \hline$\varphi$ & $\left( \pm \frac{1}{2}\right)^{3}$ & $0^{6}$ & $( \pm 1) 0^{4}$ & $( \pm 1)^{2} 0^{2}$ \\
\hline cardinality & $56 \cdot 72$ & $20 \cdot 63$ & $30 \cdot 63$ & $6 \cdot 63$ \\
\hline
\end{tabular}

According to the four types of $C_{3}$-frames, the Hirota equations for $\tau^{(n)}(x)$ are classified as follows. For each $C_{3}$-frame $\left\{ \pm a_{0}, \pm a_{1}, \pm a_{2}\right\}$ of type I with

$$
\text { (I) : } \quad\left(\phi \mid a_{0}\right)=\left(\phi \mid a_{1}\right)=\left(\phi \mid a_{2}\right)=\frac{1}{2},
$$

the Hirota equation $H\left(a_{0}, a_{1}, a_{2}\right)$ takes the form

$$
\begin{aligned}
(\mathrm{I})_{n+1 / 2}: \quad & {\left[\left(a_{1} \pm a_{2} \mid x\right)\right] \tau^{(n)}\left(x-a_{0} \delta\right) \tau^{(n+1)}\left(x+a_{0} \delta\right) } \\
& +\left[\left(a_{2} \pm a_{0} \mid x\right)\right] \tau^{(n)}\left(x-a_{1} \delta\right) \tau^{(n+1)}\left(x+a_{1} \delta\right) \\
& +\left[\left(a_{0} \pm a_{1} \mid x\right)\right] \tau^{(n)}\left(x-a_{2} \delta\right) \tau^{(n+1)}\left(x+a_{2} \delta\right)=0
\end{aligned}
$$

for $x \in H_{c+(n+1 / 2) \delta}$. This bilinear equation describes the relationship (Bäcklund transformation) between the two $\tau$-functions $\tau^{(n)}(x)$ and $\tau^{(n+1)}(x)$ on $H_{c+n \delta}$ and $H_{c+(n+1) \delta}$, 
respectively. When $\left\{ \pm a_{0}, \pm a_{1}, \pm a_{2}\right\}$ is $C_{3}$-frame of type $\mathrm{II}_{0}, \mathrm{II}_{1}, \mathrm{II}_{2}$, we choose $a_{0}, a_{1}$, $a_{2}$ so that

$$
\begin{array}{ll}
\left(\mathrm{II}_{0}\right): & \left(\phi \mid a_{0}\right)=\left(\phi \mid a_{1}\right)=\left(\phi \mid a_{2}\right)=0, \\
\left(\mathrm{II}_{1}\right): & \left(\phi \mid a_{0}\right)=1, \quad\left(\phi \mid a_{1}\right)=\left(\phi \mid a_{2}\right)=0, \\
\left(\mathrm{II}_{2}\right): & \left(\phi \mid a_{0}\right)=\left(\phi \mid a_{1}\right)=1, \quad\left(\phi \mid a_{2}\right)=0 .
\end{array}
$$

Then the corresponding Hirota equations are given by

$$
\begin{aligned}
\left(\mathrm{II}_{0}\right)_{n}: & {\left[\left(a_{1} \pm a_{2} \mid x\right)\right] \tau^{(n)}\left(x \pm a_{0} \delta\right)+\left[\left(a_{2} \pm a_{0} \mid x\right)\right] \tau^{(n)}\left(x \pm a_{1} \delta\right) } \\
& +\left[\left(a_{0} \pm a_{1} \mid x\right)\right] \tau^{(n)}\left(x \pm a_{2} \delta\right)=0, \\
\left(\mathrm{II}_{1}\right)_{n}: \quad & {\left[\left(a_{1} \pm a_{2} \mid x\right)\right] \tau^{(n-1)}\left(x-a_{0} \delta\right) \tau^{(n+1)}\left(x+a_{0} \delta\right) } \\
& =\left[\left(a_{0} \pm a_{2} \mid x\right)\right] \tau^{(n)}\left(x \pm a_{1} \delta\right)-\left[\left(a_{0} \pm a_{1} \mid x\right)\right] \tau^{(n)}\left(x \pm a_{2} \delta\right), \\
\left(\mathrm{II}_{2}\right)_{n}: \quad & {\left[\left(a_{1} \pm a_{2} \mid x\right)\right] \tau^{(n-1)}\left(x-a_{0} \delta\right) \tau^{(n+1)}\left(x+a_{0} \delta\right) } \\
& -\left[\left(a_{0} \pm a_{2} \mid x\right)\right] \tau^{(n-1)}\left(x-a_{1} \delta\right) \tau^{(n+1)}\left(x+a_{1} \delta\right) \\
= & {\left[\left(a_{1} \pm a_{0} \mid x\right)\right] \tau^{(n)}\left(x \pm a_{2} \delta\right) }
\end{aligned}
$$

for $x \in H_{c+n \delta}$. Note that the Hirota equation of type $\left(\mathrm{II}_{0}\right)_{n}$ is an equation for $\tau^{(n)}(x)$ on $H_{c+n \delta}$ only, while those of types $\left(\mathrm{II}_{1}\right)_{n}$ and $\left(\mathrm{II}_{2}\right)_{n}$ are equations among the three $\tau$-functions $\tau^{(n-1)}(x), \tau^{(n)}(x), \tau^{(n+1)}(x)$. A bilinear equation of type $\left(\mathrm{II}_{1}\right)_{n}$ can be regarded as a discrete version of the Toda equation.

For each $n \in \mathbb{Z}$, the system of 1260 Hirota equations $\left(\mathrm{II}_{0}\right)_{n}$ for $\tau^{(n)}(x)$ on $H_{c+n \delta}$ can be regarded as an ORG system of type $E_{7}$. In this way, the whole ORG system of type $E_{8}$ for $\tau(x)$ on $D_{c}$ can be regarded as an infinite chain of ORG systems of type $E_{7}$ for $\tau^{(n)}(x)$ on $H_{c+n \delta}(n \in \mathbb{Z})$.

Theorem 3.3 For an integer $n \in \mathbb{Z}$, let $\tau^{(n-1)}(x)$ and $\tau^{(n)}(x)$ be meromorphic functions on $H_{c+(n-1) \delta}$ and $H_{c+n \delta}$, respectively. Suppose that $\tau^{(n-1)}(x) \not \equiv 0$ and that the following two conditions are satisfied:

(A1): $\tau^{(n-1)}(x)$ and $\tau^{(n)}(x)$ satisfy all the bilinear equations of type $(\mathrm{I})_{n-1 / 2}$.

(A2): $\tau^{(n)}(x)$ satisfies all the bilinear equations of type $\left(\mathrm{II}_{0}\right)_{n}$.

Then there exists a unique meromorphic function $\tau^{(n+1)}(x)$ on $H_{c+(n+1) \delta}$ such that

(B): $\tau^{(n-1)}(x), \tau^{(n)}(x), \tau^{(n+1)}(x)$ satisfy all the bilinear equations of type $\left(\mathrm{II}_{1}\right)_{n}$.

Furthermore, this $\tau^{(n+1)}(x)$ satisfies the following conditions:

(C1): $\tau^{(n-1)}(x), \tau^{(n)}(x), \tau^{(n+1)}(x)$ satisfy all the bilinear equations of type $\left(\mathrm{II}_{2}\right)_{n}$.

(C2): $\tau^{(n)}(x), \tau^{(n+1)}(x)$ satisfy all the bilinear equations of type $(\mathrm{I})_{n+1 / 2}$.

(C3): $\tau^{(n+1)}(x)$ satisfies all the bilinear equations of type $\left(\mathrm{II}_{0}\right)_{n+1}$.

This theorem can be proved essentially by the same argument as that of Masuda [8, Section 3]. For completeness, we include a proof of Theorem 3.3 in Appendix A. 


\section{Hypergeometric ORG $\tau$-functions}

Keeping the notations in the previous section, we consider an ORG $\tau$-function $\tau=\tau(x)$ on

$$
D_{c}=\bigsqcup_{n \in Z} H_{c+n \delta}, \quad H_{c+n \delta}=\{x \in V \mid(\phi \mid x)=c+n \delta\} \quad(n \in \mathbb{Z}),
$$

where $c \in \mathbb{C}$. For each $n \in \mathbb{Z}$ we denote by $\tau^{(n)}=\left.\tau\right|_{H_{c+n \delta}}$ the restriction of $\tau$ to $H_{c+n \delta}$.

Definition 4.1 A meromorphic ORG $\tau$-function $\tau(x)$ on $D_{c}$ is called a hypergeometric $\tau$-function if $\tau^{(n)}(x)=0$ for $n<0$, and $\tau^{(0)}(x) \not \equiv 0$.

We now apply Theorem 3.3 for constructing hypergeometric $\tau$-functions. Since $\tau^{(-1)}(x)=0\left(x \in H_{c-\delta}\right)$, for any $C_{8}$-frame $\left\{ \pm a_{0}, \ldots, \pm a_{7}\right\}$ of type II with

$$
\left(\phi \mid a_{0}\right)=\left(\phi \mid a_{7}\right)=1, \quad\left(\phi \mid a_{i}\right)=0 \quad(i=1, \ldots, 6),
$$

$\tau^{(0)}(x)\left(x \in H_{c}\right)$ must satisfy the following three types of equations:

$$
\begin{array}{cc}
\left(\mathrm{II}_{2}\right)_{0}: & {\left[\left(a_{0} \pm a_{7} \mid x\right)\right] \tau^{(0)}\left(x \pm a_{i} \delta\right)=0} \\
\left(\mathrm{II}_{1}\right)_{0}: & {\left[\left(a_{r} \pm a_{j} \mid x\right)\right] \tau^{(0)}\left(x \pm a_{i} \delta\right)=\left[\left(a_{r} \pm a_{i} \mid x\right)\right] \tau^{(0)}\left(x \pm a_{j} \delta\right),} \\
\left(\mathrm{II}_{0}\right)_{0}: & {\left[\left(a_{j} \pm a_{k} \mid x\right)\right] \tau^{(0)}\left(x \pm a_{i} \delta\right)+\left[\left(a_{k} \pm a_{i} \mid x\right)\right] \tau^{(0)}\left(x \pm a_{j} \delta\right)} \\
+\left[\left(a_{i} \pm a_{j} \mid x\right)\right] \tau^{(0)}\left(x \pm a_{k} \delta\right)=0
\end{array}
$$

where $r=0,7$ and $i, j, k \in\{1, \ldots, 6\}$. Noting that $a_{0}+a_{7}=\phi$, in order to fulfill $\left(\mathrm{II}_{2}\right)_{0}$ for any $C_{8}$-frame of type II, we consider the case where $c=\omega \in \Omega$ is a period of $[z]$, so that $\left[\left(a_{0}+a_{7} \mid x\right)\right]=[(\phi \mid x)]=[\omega]=0$. Equations of type $\left(\mathrm{II}_{0}\right)_{0}$ follow from those of type $\left(\mathrm{II}_{0}\right)_{1}$. In fact, since

$$
\tau^{(0)}\left(x \pm a_{j} \delta\right)=\frac{\left[\left(a_{0} \pm a_{j} \mid x\right)\right]}{\left[\left(a_{0} \pm a_{k} \mid x\right)\right]} \tau^{(0)}\left(x \pm a_{k} \delta\right)
$$

for any distinct $j, k \in\{1, \ldots, 6\}$, equations $\left(\mathrm{II}_{0}\right)_{0}$ reduce to the functional equation (2.1) of $[z]$.

Theorem 4.2 Let $\omega \in \Omega$ be a period of the function $[z]$. Let $\tau^{(0)}(x)$ and $\tau^{(1)}(x)$ be nonzero meromorphic functions on $H_{\omega}$ and $H_{\omega+\delta}$, respectively. Suppose that

$$
\frac{\tau^{(0)}\left(x \pm a_{1} \delta\right)}{\tau^{(0)}\left(x \pm a_{2} \delta\right)}=\frac{\left[\left(a_{0} \pm a_{1} \mid x\right)\right]}{\left[\left(a_{0} \pm a_{2} \mid x\right)\right]} \quad\left(x \in H_{\omega}\right)
$$

for any $C_{3}$-frame $\left\{ \pm a_{0}, \pm a_{1}, \pm a_{2}\right\}$ of type $\mathrm{II}_{1}$ with $\left(\phi \mid a_{0}\right)=1$, and

$$
\begin{gathered}
{\left[\left(a_{1} \pm a_{2} \mid x\right)\right] \tau^{(0)}\left(x-a_{0} \delta\right) \tau^{(1)}\left(x+a_{0} \delta\right)+\left[\left(a_{2} \pm a_{0} \mid x\right)\right] \tau^{(0)}\left(x-a_{1} \delta\right) \tau^{(1)}\left(x+a_{1} \delta\right)} \\
+\left[\left(a_{0} \pm a_{1} \mid x\right)\right] \tau^{(0)}\left(x-a_{2} \delta\right) \tau^{(1)}\left(x+a_{2} \delta\right)=0 \quad\left(x \in H_{\omega+\delta / 2}\right)
\end{gathered}
$$

for any $C_{3}$-frame $\left\{ \pm a_{0}, \pm a_{1}, \pm a_{2}\right\}$ with $\left(\phi \mid a_{0}\right)=\left(\phi \mid a_{1}\right)=\left(\phi \mid a_{2}\right)=\frac{1}{2}$. Then there exists a unique hypergeometric $\tau$-function $\tau=\tau(x)$ on $D_{\omega}$ such that $\tau^{(n)}(x)=0$ for $n<0$ and

$$
\tau^{(0)}(x)=\tau(x) \quad\left(x \in H_{\omega}\right), \quad \tau^{(1)}(x)=\tau(x) \quad\left(x \in H_{\omega+\delta}\right) .
$$




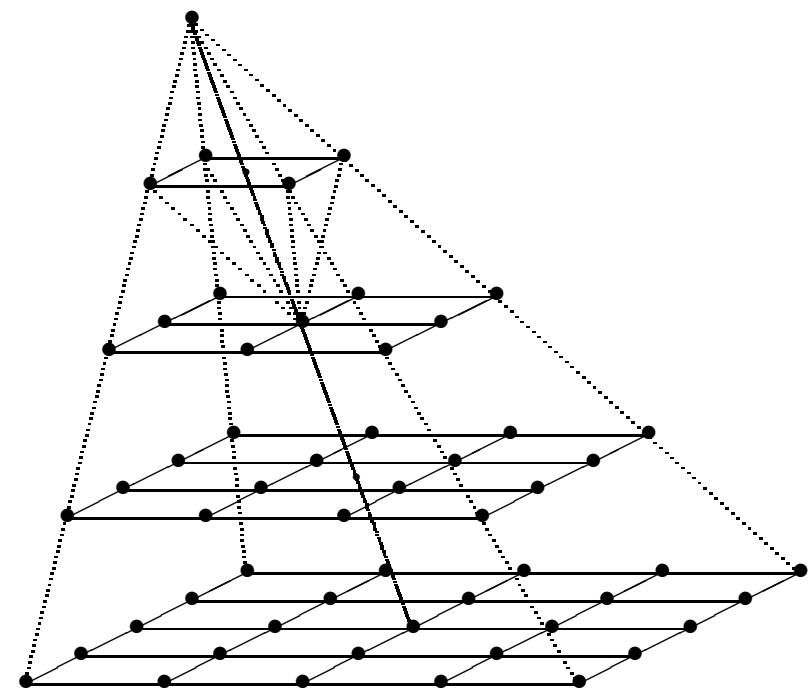

Figure 3: 2-Directional Casorati determinants

Proof: We apply Theorem 3.3 to nonzero meromorphic functions $\tau^{(n-1)}(x), \tau^{(n)}(x)$ on $H_{\omega+(n-1) \delta}, H_{c+n \delta}$, for constructing $\tau^{(n+1)}(x)$ on $H_{\omega+(n+1) \delta}$ recursively for $n=1,2, \ldots$ At each step, we need to show that the meromorphic function $\tau^{(n+1)}(x)$ determined by Theorem 3.3 is not identically zero. If $\tau^{(n+1)}(x) \equiv 0$, the bilinear equations $\left(\mathrm{II}_{2}\right)_{n}$ imply

$$
\left[\left(a_{0} \pm a_{1} \mid x\right)\right] \tau^{(n)}\left(x \pm a_{2} \delta\right)=0 \quad\left(x \in H_{\omega+n \delta}\right)
$$

for any $C_{3}$-frame $\left\{ \pm a_{0}, \pm a_{1}, \pm a_{2}\right\}$ of type $\mathrm{II}_{2}$ with $\left(\phi \mid a_{0}\right)=\left(\phi \mid a_{1}\right)=1,\left(\phi \mid a_{2}\right)=0$. Since $a_{0}+a_{1}=\phi,\left[\left(a_{0}+a_{1} \mid x\right)\right]=[\omega+n \delta] \neq 0$. Also, since $\left[\left(a_{0}-a_{1} \mid x\right)\right] \not \equiv 0$, we have $\tau^{(n)}\left(x \pm a_{2} \delta\right)=0$ and hence $\tau^{(n)}(x) \equiv 0$ on $H_{\omega+n \delta}$, contrarily to the hypothesis.

We now fix a $C_{3}$-frame $\left\{ \pm a_{0}, \pm a_{1}, \pm a_{2}\right\}$ of type $\mathrm{II}_{1}$ with $\left(\phi \mid a_{0}\right)=1,\left(\phi \mid a_{1}\right)=\left(\phi \mid a_{2}\right)=$ 0 . Then the $\tau$-functions $\tau^{(n)}$ on $H_{\omega+n \delta}$ for $n=2,3, \ldots$ are uniquely determined by the bilinear equations

$$
\begin{aligned}
\left(\mathrm{II}_{1}\right)_{n}: & {\left[\left(a_{1} \pm a_{2} \mid x\right)\right] \tau^{(n-1)}\left(x-a_{0} \delta\right) \tau^{(n+1)}\left(x+a_{0} \delta\right) } \\
& =\left[\left(a_{0} \pm a_{2} \mid x\right)\right] \tau^{(n)}\left(x \pm a_{1} \delta\right)-\left[\left(a_{0} \pm a_{1} \mid x\right)\right] \tau^{(n)}\left(x \pm a_{2} \delta\right)
\end{aligned}
$$

of Toda type. From this recursive structure, it follows that the $\tau$-functions $\tau^{(n)}(x)$ are expressed in terms of 2-directional Casorati determinants.

Theorem 4.3 Under the assumption of Theorem 4.2, suppose that $\tau^{(1)}(x)$ on $H_{\omega+\delta}$ is expressed in the form $\tau^{(1)}(x)=g^{(1)}(x) \psi(x)$ with a nonzero meromorphic function $g^{(1)}(x)$ such that

$$
\frac{g^{(1)}\left(x \pm a_{1} \delta\right)}{g^{(1)}\left(x \pm a_{2} \delta\right)}=\frac{\left[\left(a_{0} \pm a_{1} \mid x\right)\right]}{\left[\left(a_{0} \pm a_{2} \mid x\right)\right]} \quad\left(x \in H_{\omega+\delta}\right) .
$$


for a $C_{3}$-frame $\left\{ \pm a_{0}, \pm a_{1}, \pm a_{2}\right\}$ of type $\mathrm{II}_{1}$ with $\left(\phi \mid a_{0}\right)=1,\left(\phi \mid a_{1}\right)=\left(\phi \mid a_{2}\right)=0$. Then the components $\tau^{(n)}(x)$ of the hypergeometric $\tau$-function $\tau(x)$ are expressed as follows in terms of 2-directional Casorati determinants:

$$
\tau^{(n)}(x)=g^{(n)}(x) K^{(n)}(x), \quad K^{(n)}(x)=\operatorname{det}\left(\psi_{i j}^{(n)}(x)\right)_{i, j=1}^{n} \quad\left(x \in H_{\omega+n \delta}\right)
$$

for $n=0,1,2, \ldots$, where

$$
\psi_{i j}^{(n)}(x)=\psi\left(x-(n-1) a_{0} \delta+(n+1-i-j) a_{1} \delta+(j-i) a_{2} \delta\right)
$$

for $i, j=1, \ldots, n$. The gauge factors $g^{(n)}(x)$ are determined inductively from $g^{(0)}(x)=$ $\tau^{(0)}(x)$ and $g^{(1)}(x)$ by

$$
\frac{g^{(n-1)}\left(x-a_{0} \delta\right) g^{(n+1)}\left(x+a_{0} \delta\right)}{g^{(n)}\left(x \pm a_{1} \delta\right)}=\frac{\left[\left(a_{0} \pm a_{2} \mid x\right)\right]}{\left[\left(a_{1} \pm a_{2} \mid x\right)\right]} \quad\left(x \in H_{\omega+n \delta}\right)
$$

for $n=1,2, \ldots$

Lemma 4.4 The gauge factors $g^{(n)}(x)\left(x \in H_{\omega+n \delta}\right)$ defined by (4.13) satisfy

$$
\frac{g^{(n)}\left(x \pm a_{1} \delta\right)}{g^{(n)}\left(x \pm a_{2} \delta\right)}=\frac{\left[\left(a_{0} \pm a_{1} \mid x\right)\right]}{\left[\left(a_{0} \pm a_{2} \mid x\right)\right]} \quad(n=0,1,2, \ldots) .
$$

Proof: Formulas (4.14) for $n=0,1$ are included in the assumption for $g^{(0)}(x)=\tau^{(0)}(x)$ and $g^{(1)}(x)$. For $n=1,2, \ldots$, we show inductively that $g^{(n+1)}(x)$ defined by (4.13) satisfies this condition. From (4.14) for $g^{(n)}(x)$, we have

$$
g^{(n+1)}\left(x+a_{0} \delta\right)=\frac{\left[\left(a_{0} \pm a_{2} \mid x\right)\right]}{\left[\left(a_{1} \pm a_{2} \mid x\right)\right]} \frac{g^{(n)}\left(x \pm a_{1} \delta\right)}{g^{(n-1)}\left(x-a_{0} \delta\right)}=\frac{\left[\left(a_{0} \pm a_{1} \mid x\right)\right]}{\left[\left(a_{1} \pm a_{2} \mid x\right)\right]} \frac{g^{(n)}\left(x \pm a_{2} \delta\right)}{g^{(n-1)}\left(x-a_{0} \delta\right)}
$$

and hence

$$
\begin{aligned}
g^{(n+1)}(x) & =\frac{\left[\left(a_{0} \pm a_{2} \mid x\right)-\delta\right]}{\left[\left(a_{1} \pm a_{2} \mid x\right)\right]} \frac{g^{(n)}\left(x-a_{0} \delta \pm a_{1} \delta\right)}{g^{(n-1)}\left(x-2 a_{0} \delta\right)} \\
& =\frac{\left[\left(a_{0} \pm a_{1} \mid x\right)-\delta\right]}{\left[\left(a_{1} \pm a_{2} \mid x\right)\right]} \frac{g^{(n)}\left(x-a_{0} \delta \pm a_{2} \delta\right)}{g^{(n-1)}\left(x-2 a_{0} \delta\right)}
\end{aligned}
$$

From these two expressions of $g^{(n+1)}(x)$ we obtain

$$
\begin{aligned}
& g^{(n+1)}\left(x \pm a_{2} \delta\right)=\frac{\left[\left(a_{0} \pm a_{2} \mid x\right)\right]\left[\left(a_{0} \pm a_{2} \mid x\right)-2 \delta\right]}{\left[\left(a_{1} \pm a_{2} \mid x\right) \pm \delta\right]} \frac{g^{(n)}\left(x-a_{0} \delta \pm a_{1} \delta \pm a_{2} \delta\right)}{g^{(n-1)}\left(x-2 a_{0} \delta \pm a_{2} \delta\right)} \\
& g^{(n+1)}\left(x \pm a_{1} \delta\right)=\frac{\left[\left(a_{0} \pm a_{1} \mid x\right)\right]\left[\left(a_{0} \pm a_{1} \mid x\right)-2 \delta\right]}{\left[\left(a_{1} \pm a_{2} \mid x\right) \pm \delta\right]} \frac{g^{(n)}\left(x-a_{0} \delta \pm a_{1} \delta \pm a_{2} \delta\right)}{g^{(n-1)}\left(x-2 a_{0} \delta \pm a_{1} \delta\right)}
\end{aligned}
$$

Then by (4.14) for $g^{(n-1)}(x)$ we obtain

$$
\begin{aligned}
\frac{g^{(n+1)}\left(x \pm a_{1} \delta\right)}{g^{(n+1)}\left(x \pm a_{2} \delta\right)} & =\frac{\left[\left(a_{0} \pm a_{1} \mid x\right)\right]\left[\left(a_{0} \pm a_{1} \mid x\right)-2 \delta\right]}{\left[\left(a_{0} \pm a_{2} \mid x\right)\right]\left[\left(a_{0} \pm a_{2} \mid x\right)-2 \delta\right]} \frac{g^{(n-1)}\left(x-2 a_{0} \delta \pm a_{2} \delta\right)}{g^{(n-1)}\left(x-2 a_{0} \delta \pm a_{1} \delta\right)} \\
& =\frac{\left[\left(a_{0} \pm a_{1} \mid x\right)\right]}{\left[\left(a_{0} \pm a_{2} \mid x\right)\right]}
\end{aligned}
$$


as desired.

Proof of Theorem 4.3]: Using the gauge factors $g^{(n)}(x)$ defined as above, we set

$$
\tau^{(0)}(x)=g^{(0)}(x), \quad \tau^{(1)}(x)=g^{(1)}(x) \psi(x),
$$

and define $K^{(n)}(x)$ by

$$
\tau^{(n)}(x)=g^{(n)}(x) K^{(n)}(x) \quad(n=0,1,2, \ldots) .
$$

Then the bilinear equation (4.9) is written as

$$
\begin{aligned}
& \quad\left[\left(a_{1} \pm a_{2} \mid x\right)\right] g^{(n-1)}\left(x-a_{0} \delta\right) g^{(n+1)}\left(x+a_{0} \delta\right) \\
& \quad \cdot K^{(n-1)}\left(x-a_{0} \delta\right) K^{(n+1)}\left(x+a_{0} \delta\right) \\
& =\left[\left(a_{0} \pm a_{2} \mid x\right)\right] g^{(n)}\left(x \pm a_{1} \delta\right) K^{(n)}\left(x \pm a_{1} \delta\right) \\
& \quad-\left[\left(a_{0} \pm a_{1} \mid x\right)\right] g^{(n)}\left(x \pm a_{2} \delta\right) K^{(n)}\left(x \pm a_{2} \delta\right) .
\end{aligned}
$$

By Lemma 4.4, we have

$$
\begin{aligned}
& {\left[\left(a_{1} \pm a_{2} \mid x\right)\right] g^{(n-1)}\left(x-a_{0} \delta\right) g^{(n+1)}\left(x+a_{0} \delta\right)} \\
& =\left[\left(a_{0} \pm a_{2} \mid x\right)\right] g^{(n)}\left(x \pm a_{1} \delta\right)=\left[\left(a_{0} \pm a_{1} \mid x\right)\right] g^{(n)}\left(x \pm a_{2} \delta\right) .
\end{aligned}
$$

Therefore, the main factors $K^{(n)}(x)$ are determined by

$$
K^{(n-1)}\left(x-a_{0} \delta\right) K^{(n+1)}\left(x+a_{0} \delta\right)=K^{(n)}\left(x \pm a_{1} \delta\right)-K^{(n)}\left(x \pm a_{2} \delta\right)
$$

for $n=1,2, \ldots$ starting from $K^{(0)}(x)=1, K^{(1)}(x)=\psi(x)$. For example, we have

$$
\begin{aligned}
& K^{(2)}\left(x+a_{0} \delta\right)=\psi\left(x \pm a_{1} \delta\right)-\psi\left(x \pm a_{2} \delta\right)=\operatorname{det}\left[\begin{array}{cc}
\psi\left(x+a_{1} \delta\right) & \psi\left(x+a_{2} \delta\right) \\
\psi\left(x-a_{2} \delta\right) & \psi\left(x-a_{1} \delta\right)
\end{array}\right] \\
& K^{(3)}\left(x+2 a_{0} \delta\right)=\operatorname{det}\left[\begin{array}{ccc}
\psi\left(x+2 a_{1} \delta\right) & \psi\left(x+a_{1} \delta+a_{2} \delta\right) & \psi\left(x+2 a_{2} \delta\right) \\
\psi\left(x+a_{1} \delta-a_{2} \delta\right) & \psi(x) & \psi\left(x-a_{1} \delta+a_{2} \delta\right) \\
\psi\left(x-2 a_{2} \delta\right) & \psi\left(x-a_{1} \delta-a_{2} \delta\right) & \psi\left(x-2 a_{1} \delta\right)
\end{array}\right] .
\end{aligned}
$$

In general, this recurrence (4.23) for $K^{(n)}(x)$ is solved by the Lewis Carroll formula for the Casorati determinants with respect to the two directions $a_{1}+a_{2}$ and $a_{1}-a_{2}$. Namely, for $n=1,2, \ldots$, we have

$$
K^{(n)}\left(x+(n-1) a_{0} \delta\right)=\operatorname{det}\left(\psi\left(x+(n+1-i-j) a_{1} \delta+(j-i) a_{2} \delta\right)\right)_{i, j=1}^{n}
$$


with the vectors $v_{i j}=(n+1-i-j) a_{1}+(j-i) a_{2}(i, j=1, \ldots, n)$ arranged as follows.

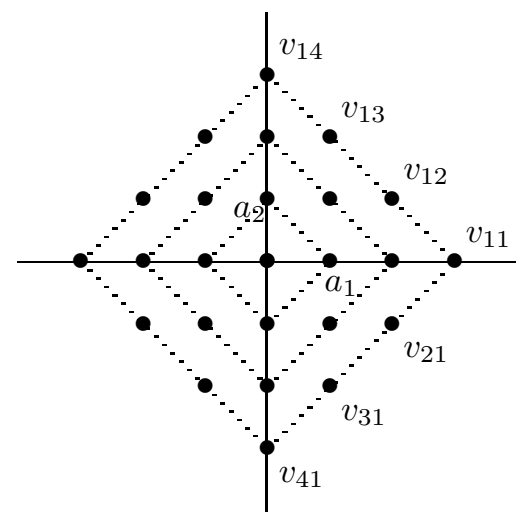

This implies the expression (4.11) for $K^{(n)}(x)$.

\section{$5 \quad$ Elliptic hypergeometric integrals}

In this section, we recall fundamental facts concerning the elliptic hypergeometric integrals of Spiridonov [16, 17] and Rains [12, 13].

Fixing two bases $p, q \in \mathbb{C}^{*}=\mathbb{C} \backslash\{0\}$ with $|p|<1,|q|<1$, we use the multiplicative notations

$$
\theta(z ; p)=(z ; p)_{\infty}(p / z ; p)_{\infty}, \quad(z ; p)_{\infty}=\prod_{i=0}^{\infty}\left(1-p^{i} z\right)
$$

for the Jacobi theta function, and

$$
\Gamma(z ; p, q)=\frac{(p q / z ; p, q)_{\infty}}{(z ; p, q)_{\infty}}, \quad(z ; p, q)_{\infty}=\prod_{i, j=0}^{\infty}\left(1-p^{i} q^{j} z\right)
$$

for the Ruijsenaars elliptic gamma function. These functions satisfy the functional equations

$$
\begin{aligned}
& \theta(p z ; p)=-z^{-1} \theta(z ; p), \quad \theta(p / z ; p)=\theta(z ; p), \\
& \Gamma(q z ; p, q)=\theta(z ; p) \Gamma(z ; p, q), \quad \Gamma(p q / u ; p, q)=\Gamma(u ; p, q)^{-1} .
\end{aligned}
$$

The multiplicative theta function $\theta(z ; p)$ satisfies the three-term relation

$$
c \theta\left(b c^{ \pm 1} ; p\right) \theta\left(a z^{ \pm 1} ; p\right)+a \theta\left(c a^{ \pm 1} ; p\right) \theta\left(b z^{ \pm 1} ; p\right)+b \theta\left(a b^{ \pm 1} ; p\right) \theta\left(c z^{ \pm 1} ; p\right)=0,
$$

corresponding to (2.1). Here we have used the abbreviation $\theta\left(a b^{ \pm 1} ; p\right)=\theta(a b ; p) \theta\left(a b^{-1} ; p\right)$ to refer to the product of two factors with different signs. Note also that

$$
\frac{1}{\Gamma\left(z^{ \pm 1} ; p, q\right)}=\left(1-z^{ \pm 1}\right)\left(p z^{ \pm 1} ; p\right)_{\infty}\left(q z^{ \pm 1} ; q\right)_{\infty}=-z^{-1} \theta(z ; p) \theta(z ; q)
$$


Following Spiridonov [16, 17, we consider the elliptic hypergeometric integral $I(u ; p, q)$ in eight variables $u=\left(u_{0}, u_{1}, \ldots, u_{7}\right)$ defined by

$$
I(u ; p, q)=\frac{(p ; p)_{\infty}(q ; q)_{\infty}}{4 \pi \sqrt{-1}} \int_{C} \frac{\prod_{k=0}^{7} \Gamma\left(u_{k} z^{ \pm 1} ; p, q\right)}{\Gamma\left(z^{ \pm 2} ; p, q\right)} \frac{d z}{z} .
$$

Here we assume that $u=\left(u_{0}, u_{1}, \ldots, u_{7}\right)$ is generic in the sense that $u_{k} u_{l} \notin p^{-\mathbb{N}} q^{-\mathbb{N}}$ for any $k, l \in\{0,1, \ldots, 7\}(\mathbb{N}=\{0,1,2, \ldots\})$. This condition is equivalent to saying that the two sets

$$
\begin{aligned}
S_{0} & =\left\{p^{i} q^{j} u_{k} \mid i, j \in \mathbb{N}, k \in\{0,1, \ldots, 7\}\right\}, \\
S_{\infty} & =\left\{p^{-i} q^{-j} u_{k}^{-1} \mid i, j \in \mathbb{N}, k \in\{0,1, \ldots, 7\}\right\}
\end{aligned}
$$

of possible poles of the integrand are disjoint. For the contour $C$ we take a homology cycle in $\mathbb{C}^{*} \backslash\left(S_{0} \cup S_{\infty}\right)$ such that $n(C, a)=1$ for all $a \in S_{0}$ and $n(C, a)=0$ for all $a \in S_{\infty}$, where $n(C ; a)$ stands for the winding number of $C$ around $z=a$. Note also that, if $\left|u_{k}\right|<1(k=0,1, \ldots, 7)$, one can take the unit circle $|z|=1$ oriented positively as the cycle $C$.

The following transformation formulas are due to Spiridonov [16] and Rains [13].

Theorem 5.1 Suppose that the parameters $u=\left(u_{0}, u_{1}, \ldots, u_{7}\right)$ satisfy the balancing condition $u_{0} u_{1} \cdots u_{7}=p^{2} q^{2}$. Then the following transformation formulas hold:

$$
\begin{aligned}
& \text { (1) } I(u ; p, q)=I(\widetilde{u} ; p, q) \prod_{0 \leq i<j \leq 3} \Gamma\left(u_{i} u_{j} ; p, q\right) \prod_{4 \leq i<j \leq 7} \Gamma\left(u_{i} u_{j} ; p, q\right), \\
& \widetilde{u}=\left(\widetilde{u}_{0}, \widetilde{u}_{1}, \ldots, \widetilde{u}_{7}\right), \quad \widetilde{u}_{i}= \begin{cases}u_{i} \sqrt{p q / u_{0} u_{1} u_{2} u_{3}} & (i=0,1,2,3), \\
u_{i} \sqrt{p q / u_{4} u_{5} u_{6} u_{7}} & (i=4,5,6,7),\end{cases}
\end{aligned}
$$

and

$$
\begin{aligned}
& I(u ; p, q)=I(\widehat{u} ; p, q) \prod_{0 \leq i<j \leq 7} \Gamma\left(u_{i} u_{j} ; p, q\right), \\
& \widehat{u}=\left(\widehat{u}_{0}, \ldots, \widehat{u}_{7}\right), \quad \widehat{u}_{i}=\sqrt{p q} / u_{i} \quad(i=0,1, \ldots, 7) .
\end{aligned}
$$

Note that, if the parameters $u=\left(u_{0}, u_{1}, \ldots, u_{7}\right)$ satisfy $u_{0} u_{1} \cdots u_{7}=p^{2} q^{2}$ and $\left|u_{k}\right|=$ $|p q|^{\frac{1}{4}}(k=0,1, \ldots, 7)$, then both $\widetilde{u}$ and $\widehat{u}$ satisfy the two conditions as well.

Taking another base $r \in \mathbb{C}^{*}$ with $|r|<1$, we set

$$
\Psi(u ; p, q, r)=I(u ; p, q) \prod_{0 \leq i<j \leq 7} \Gamma\left(u_{i} u_{j} ; p, q, r\right),
$$

where

$$
\begin{aligned}
\Gamma(z ; p, q, r) & =(z ; p, q, r)_{\infty}(p q r / z ; p, q, r)_{\infty} \\
(z ; p, q, r)_{\infty} & =\prod_{i, j, k=0}^{\infty}\left(1-p^{i} q^{j} r^{k} z\right) .
\end{aligned}
$$


Note that

$$
\Gamma(r z ; p, q, r)=\Gamma(z ; p, q) \Gamma(z ; p, q, r), \quad \Gamma(p q r / z ; p, q, r)=\Gamma(z ; p, q, r) .
$$

Proposition 5.2 Under the condition $u_{0} u_{1} \cdots u_{7}=p^{2} q^{2}$, the function $\Psi(u ; p, q, r)$ defined by (5.10) is invariant with respect to the transformations $u \rightarrow \widetilde{u}$ and $u \rightarrow \widehat{u}$.

Proof: When $\{i, j, k, l\}=\{0,1,2,3\}$ or $\{4,5,6,7\}$, one has

$$
\begin{aligned}
\Gamma\left(\widetilde{u}_{i} \widetilde{u}_{j} ; p, q, r\right) & =\Gamma\left(p q / u_{k} u_{l} ; p, q, r\right)=\Gamma\left(r u_{k} u_{l} ; p, q, r\right) \\
& =\Gamma\left(u_{k}, u_{l} ; p, q\right) \Gamma\left(u_{k} u_{l} ; p, q, r\right) .
\end{aligned}
$$

Also, for distinct $i, j \in\{0,1, \ldots, 7\}$,

$$
\begin{aligned}
\Gamma\left(\widehat{u}_{i} \widehat{u}_{j} ; p, q, r\right) & =\Gamma\left(p q / u_{i} u_{j} ; p, q, r\right)=\Gamma\left(r u_{i} u_{j} ; p, q, r\right) \\
& =\Gamma\left(r u_{i} u_{j} ; p, q\right) \Gamma\left(u_{i} u_{j} ; p, q, r\right) .
\end{aligned}
$$

Using these formulas, Theorem 5.1 can be reformulated as

$$
\Psi(u ; p, q, r)=\Psi(\widetilde{u} ; p, q, r)=\Psi(\widehat{u} ; p, q, r)
$$

under the condition $u_{0} u_{1} \cdots u_{7}=p^{2} q^{2}$.

Returning to the elliptic hypergeometric integral (5.6), we notice that the integrand

$$
H(z, u ; p, q)=\frac{\prod_{k=0}^{7} \Gamma\left(u_{k} z^{ \pm 1} ; p, q\right)}{\Gamma\left(z^{ \pm 2} ; p, q\right)}
$$

satisfies

$$
T_{q, u_{k}} H(z, u ; p, q)=\theta\left(u_{k} z^{ \pm 1} ; p\right) H(z, u ; p, q)
$$

with respect to the $q$-shift operator $T_{q, u_{k}}$ in $u_{k}(k \in\{0,1, \ldots, 7\})$ :

$$
T_{q, u_{k}} f\left(u_{0}, u_{1}, \ldots, u_{7}\right)=f\left(u_{0}, \ldots, q u_{k}, \ldots, u_{7}\right) .
$$

Hence, by the functional equation (5.4) we have

$$
\left(u_{k} \theta\left(u_{j} u_{k}^{ \pm 1} ; p\right) T_{q, u_{i}}+u_{i} \theta\left(u_{k} u_{i}^{ \pm 1} ; p\right) T_{q, u_{j}}+u_{j} \theta\left(u_{i} u_{j}^{ \pm 1} p\right) T_{q, u_{k}}\right) H(z, u ; p, q)=0
$$

for any triple $i, j, k \in\{0,1, \ldots, 7\}$. Passing to the integral, we obtain the following contiguity relations for the elliptic hypergeometric integral.

Proposition 5.3 The elliptic hypergeometric integral (5.6) satisfies the three-term relation

$$
\left(u_{k} \theta\left(u_{j} u_{k}^{ \pm 1} ; p\right) T_{q, u_{i}}+u_{i} \theta\left(u_{k} u_{i}^{ \pm 1} ; p\right) T_{q, u_{j}}+u_{j} \theta\left(u_{i} u_{j}^{ \pm 1} ; p\right) T_{q, u_{k}}\right) I(u ; p, q)=0
$$

for any triple $i, j, k \in\{0,1, \ldots, 7\}$. 
It is known that the elliptic hypergeometric integral $I(u ; p, q)$ of (5.6) gives rise to terminating elliptic hypergeometric series in the special cases where $p q / u_{k} u_{l}=p^{-M} q^{-N}$ for distinct $k, l \in\{0,1, \ldots, 7\}$ and $M, N \in \mathbb{N}$ (see for example Komori [7]). Here we give a remark on the case where $p q / u_{0} u_{7}=q^{-N}$ for simplicity. We use the notation of very well-poised elliptic hypergeometric series

$$
\begin{aligned}
& { }_{12} V_{11}\left(a_{0} ; a_{1}, \ldots, a_{7} ; q, p\right)=\sum_{k=0}^{\infty} \frac{\theta\left(q^{2 k} a_{0} ; p\right)}{\theta\left(a_{0} ; p\right)}\left(\prod_{i=0}^{7} \frac{\theta\left(a_{i} ; p ; q\right)_{k}}{\theta\left(q a_{0} / a_{i} ; p ; q\right)_{k}}\right) q^{k} \\
& \theta(z ; p ; q)_{k}=\frac{\Gamma\left(q^{k} z ; p, q\right)}{\Gamma(z ; p, q)}=\theta(z ; p) \theta(q z ; p) \cdots \theta\left(q^{k-1} z ; p\right) \quad(k=0,1,2, \ldots),
\end{aligned}
$$

assuming that $a_{i} \in p^{\mathbb{Z}} q^{-N}$ for some $i \in\{0,1, \ldots, 7\}$ and $N=0,1,2, \ldots$

Proposition 5.4 Under the balancing condition $u_{0} u_{1} \cdots u_{7}=q^{2}$, we assume either $q / u_{0} u_{i}=q^{-N}$ for some $i \in\{1, \ldots, 6\}$ or $q / u_{0} u_{7}=p q^{-N}$, where $N=0,1,2, \ldots$ Then we have

$$
\begin{aligned}
& I\left(p u_{0}, u_{1}, \ldots, u_{6}, p u_{7} ; p, q\right) \\
& =\prod_{1 \leq k<l \leq 6} \Gamma\left(u_{k} u_{l} ; p, q\right) \frac{\Gamma\left(q^{2} / u_{0}^{2} ; p, q\right) \Gamma\left(u_{0} / u_{7} ; p, q\right)}{\prod_{k=1}^{6} \Gamma\left(q u_{i} / u_{0} ; p, q\right) \Gamma\left(q / u_{i} u_{7} ; p, q\right)} \\
& \quad \cdot{ }_{12} V_{11}\left(q / u_{0}^{2} ; q / u_{0} u_{1}, \ldots, q / u_{0} u_{6}, q / u_{0} u_{7} ; q, p\right) .
\end{aligned}
$$

Sketch of proof: Under the balancing condition $u_{0} u_{1} \cdots u_{7}=p^{2} q^{2}$, we set $t=$ $\left(t_{0}, t_{1}, \ldots, t_{7}\right), t_{i}=\sqrt{p q} / u_{i}(i=0,1, \ldots, 7)$, so that

$$
I(u ; p, q)=I(t ; p, q) \prod_{0 \leq k<l \leq 7} \Gamma\left(u_{k} u_{l} ; p, q\right)=\frac{I(t ; p, q)}{\prod_{0 \leq k<l \leq 7} \Gamma\left(t_{k} t_{l} ; p, q\right)} .
$$

We investigate the behavior of the both sides in the limit as $p q / u_{0} u_{7} \rightarrow q^{-N}$, namely, $t_{0} t_{7} \rightarrow q^{-N}$. In this limit $u_{7} \rightarrow p q^{1+N} / u_{0}, t_{7} \rightarrow q^{-N} / t_{0}$, the integral $I(u ; p, q)$ on the left-hand side has a finite limit, while $I(t ; p, q)$ gives rise to singularities due to pinching of the contour at

$$
q^{k} t_{7} \rightarrow q^{-N+k} / t_{0}, \quad q^{-k} t_{7}^{-1} \rightarrow q^{N-k} t_{0} \quad(k=0,1, \ldots, N) .
$$

By the residue calculus around these points, we can compute the limit

$$
\begin{aligned}
\lim _{t_{7} \rightarrow q^{-N} t_{0}} \frac{I(t ; p, q)}{\prod_{0 \leq k<l \leq 7} \Gamma\left(t_{k} t_{l} ; p, q\right)} \\
=\frac{1}{\prod_{0 \leq i<j \leq 6} \Gamma\left(t_{i} t_{j} ; p, q\right)} \prod_{\nu=0}^{N-1} \frac{\prod_{i=1}^{6} \theta\left(q^{-N+\nu} t_{i} / t_{0} ; p\right)}{\theta\left(q^{-N+\nu} / t_{0}^{2} ; p\right)} \\
\quad \cdot{ }_{12} V_{11}\left(p t_{0}^{2} ; t_{0} t_{1}, \ldots, t_{0} t_{6}, p q^{-N} ; q, p\right) .
\end{aligned}
$$


Hence, under the conditions $u_{0} u_{1} \cdots u_{7}=p^{2} q^{2}$ and $p q / u_{0} u_{7}=q^{-N}$, we obtain

$$
\begin{aligned}
I(u ; p, q)= & \Gamma\left(p^{2} q^{2} / u_{0}^{2} ; p, q\right) \prod_{i=1}^{7} \Gamma\left(u_{0} / u_{i} ; p, q\right) \prod_{1 \leq i<j \leq 7} \Gamma\left(u_{i} u_{j} ; p, q\right) \\
& \cdot{ }_{12} V_{11}\left(p^{2} q / u_{0}^{2} ; p q / u_{0} u_{1}, \ldots, p q / u_{0} u_{6}, p^{2} q / u_{0} u_{7} ; q, p\right) .
\end{aligned}
$$

Noting that ${ }_{12} V_{11}\left(a_{0} ; a_{1}, \ldots, a_{7} ; q, p\right)$ is invariant under the $p$-shift operator $T_{p, a_{i}} T_{p, a_{j}}^{-1}$ for distinct $i, j \in\{1, \ldots, 7\}$, we see that the same formula (5.26) holds if we replace the condition " $p q / u_{0} u_{7}=q^{-N "}$ " by " $p q / u_{0} u_{i}=q^{-N}$ for some $i \in\{1, \ldots, 6\}$ ". Then, replacing $u_{0}, u_{7}$ by $p u_{0}, p u_{7}$ respectively, we obtain (5.22).

\section{$6 \quad W\left(E_{7}\right)$-invariant hypergeometric $\tau$-function}

In the following, we present an explicit hypergeometric $\tau$-function for the ORG system of type $E_{8}$ in terms of elliptic hypergeometric integrals.

We denote by $x=\left(x_{0}, x_{1}, \ldots, x_{7}\right)$ the canonical coordinates of $V=\mathbb{C}^{8}$ so that

$$
x=\left(x_{0}, x_{1}, \ldots, x_{7}\right)=x_{0} v_{0}+\cdots+x_{7} v_{7} ; \quad x_{i}=\left(v_{i} \mid x\right) \quad(i=0,1, \ldots, 7) .
$$

Note that the highest root $\phi=\frac{1}{2}\left(v_{0}+v_{1}+\cdots+v_{7}\right)$ of $\Delta\left(E_{8}\right)$ corresponds to the linear function

$$
(\phi \mid x)=\frac{1}{2}\left(x_{0}+x_{1}+\cdots+x_{7}\right) .
$$

We relate the additive coordinates $x=\left(x_{0}, x_{1}, \ldots, x_{7}\right)$ and the multiplicative coordinates $u=\left(u_{0}, u_{1}, \ldots, u_{7}\right)$ through $u_{i}=e\left(x_{i}\right)=e^{2 \pi \sqrt{-1} x_{i}}(i=0,1, \ldots, 7)$. We also use the notation of exponential functions

$$
u^{\lambda}=e((\lambda \mid x)) \quad(\lambda \in P),
$$

so that $u^{v_{i}}=u_{i}(i=0,1, \ldots, 7)$ and $u^{\phi}=\left(u_{0} u_{1} \cdots u_{7}\right)^{\frac{1}{2}}$.

We now consider the case where the fundamental function $[\zeta](\zeta \in \mathbb{C})$ is quasiperiodic with respect to the $\Omega=\mathbb{Z} 1 \oplus \mathbb{Z} \varpi, \operatorname{Im}(\varpi)>0$, and is expressed as

$$
[\zeta]=z^{-\frac{1}{2}} \theta(z ; p), \quad z=e(\zeta)
$$

with base $p=e(\varpi),|p|<1$. This function has the quasi-periodicity

$$
[\zeta+1]=-[\zeta], \quad[\zeta+\varpi]=-e\left(-\zeta-\frac{\varpi}{2}\right)[\zeta]
$$

and hence $\eta_{1}=0, \eta_{\varpi}=-1$. Note also that

$$
[\alpha \pm \beta]=a^{-1} \theta\left(a b^{ \pm 1} ; p\right) \quad(a=e(\alpha), b=e(\beta)),
$$

and that the three-term relation (5.4) for $\theta(z ; p)$ corresponds to (2.1) for $[\zeta]$. As to the constant $\delta \in \mathbb{C}$, we assume $\operatorname{Im}(\delta)>0$, and set $q=e(\delta)$ so that $|q|<1$. 
As in Section 1, we take the simple roots

$$
\alpha_{0}=\phi-v_{0}-v_{1}-v_{2}-v_{3}, \quad \alpha_{j}=v_{j}-v_{j+1} \quad(j=1, \ldots, 6)
$$

for the root system $\Delta\left(E_{7}\right)$. Since $v_{1}-v_{0}$ is the highest root, we see that the Weyl group $W\left(E_{7}\right)$ is generated by $\mathfrak{S}_{8}=\left\langle r_{v_{j}-v_{j+1}}(j=0,1, \ldots, 6)\right\rangle$ and the reflection $s_{0}=$ $r_{\alpha_{0}}$ by $\alpha_{0}=r_{\phi-v_{0}-v_{1}-v_{2}-v_{3}}$. The symmetric group $\mathfrak{S}_{8}$ acts on the coordinates $x=$ $\left(x_{0}, x_{1}, \ldots, x_{7}\right)$ and $u=\left(u_{0}, u_{1}, \ldots, u_{7}\right)$ through the permutation of indices, while $s_{0}$ acts on the additive coordinates as

$$
s_{0}\left(x_{i}\right)= \begin{cases}x_{i}+\frac{1}{2}\left((\phi \mid x)-x_{0}-x_{1}-x_{2}-x_{3}\right) & (i=0,1,2,3), \\ x_{i}+\frac{1}{2}\left((\phi \mid x)-x_{4}-x_{5}-x_{6}-x_{7}\right) & (i=4,5,6,7),\end{cases}
$$

and on the multiplicative coordinates $u_{0}, u_{1}, \ldots, u_{7}$ as

$$
s_{0}\left(u_{i}\right)= \begin{cases}u_{i}\left(u^{\phi} / u_{0} u_{1} u_{2} u_{3}\right)^{\frac{1}{2}} & (i=0,1,2,3), \\ u_{i}\left(u^{\phi} / u_{4} u_{5} u_{6} u_{7}\right)^{\frac{1}{2}} & (i=4,5,6,7) .\end{cases}
$$

We now restrict the coordinates $x_{i}$ and $u_{i}$ to the level set

$$
H_{\kappa}=\left\{x \in V \mid(\phi \mid x)=\frac{1}{2}\left(x_{0}+x_{1}+\cdots+x_{7}\right)=\kappa\right\} \quad(\kappa \in \mathbb{C})
$$

so that $u^{\phi}=\left(u_{0} u_{1} \cdots u_{7}\right)^{\frac{1}{2}}=e(\kappa)$. Then the action of $s_{0}$ is given by

$$
s_{0}\left(x_{i}\right)= \begin{cases}x_{i}+\frac{1}{2}\left(\kappa-x_{0}-x_{1}-x_{2}-x_{3}\right) & (i=0,1,2,3), \\ x_{i}+\frac{1}{2}\left(\kappa-x_{4}-x_{5}-x_{6}-x_{7}\right) & (i=4,5,6,7) .\end{cases}
$$

and by

$$
s_{0}\left(u_{i}\right)= \begin{cases}u_{i} \sqrt{e(\kappa) / u_{0} u_{1} u_{2} u_{3}} & (i=0,1,2,3), \\ u_{i} \sqrt{e(\kappa) / u_{4} u_{5} u_{6} u_{7}} & (i=4,5,6,7) .\end{cases}
$$

respectively. Suppose $\kappa=\varpi+\delta$ so that $e(\kappa)=e(\varpi+\delta)=p q$. In this case, we have $u_{0} u_{1} \cdots u_{7}=p^{2} q^{2}$ on $H_{\varpi+\delta}$, and the action of $s_{0}$ coincides with the transformation $u_{i} \rightarrow \widetilde{u}_{i}$ in (5.8). Proposition 5.2 thus implies that the function

$$
\Psi(u ; p, q, r)=I(u ; p, q) \prod_{0 \leq k<l \leq 7} \Gamma\left(u_{k} u_{l} ; p, q, r\right)
$$

regarded as a function on $H_{\varpi+\delta}, u^{\phi}=p q$, is invariant under the action of $s_{0}$. Since $\Psi(u ; p, q, r)$ is manifestly symmetric with respect to $u=\left(u_{0}, u_{1}, \ldots, u_{7}\right)$, we see that $\Psi(u ; p, q, r)$ is a $W\left(E_{7}\right)$-invariant meromorphic function on $H_{\varpi+\delta}$. We remark that the transformation $u_{i} \rightarrow \widehat{u}_{i}$ in (5.9) coincides with the action of

$$
w=r_{07} r_{12} r_{34} r_{56} r_{0127} r_{0347} r_{0567} \in W\left(E_{7}\right),
$$

where $r_{i j}=r_{v_{i}-v_{j}}$ and $r_{i j k l}=r_{\phi-v_{i}-v_{j}-v_{k}-v_{l}}$. This means that the transformation formula (2) of Theorem 5.1 follows from (1). 
We rewrite the contiguity relations of (5.20) as

$$
\begin{gathered}
u_{j}^{-1} \theta\left(u_{j} u_{k}^{ \pm 1} ; p\right) u_{i}^{-1} T_{q, u_{i}} I(u ; p, q)+u_{k}^{-1} \theta\left(u_{k} u_{i}^{ \pm 1} ; p\right) u_{j}^{-1} T_{q, u_{j}} I(u ; p, q) \\
+u_{i}^{-1} \theta\left(u_{i} u_{j}^{ \pm 1} ; p\right) u_{k}^{-1} T_{q, u_{k}} I(u ; p, q)=0 .
\end{gathered}
$$

Since $u_{i}^{-1} \theta\left(u_{i} u_{j}^{ \pm 1} ; p\right)=\left[x_{i} \pm x_{j}\right]$, this means that

$$
\begin{gathered}
{\left[x_{j} \pm x_{k}\right] u_{i}^{-1} T_{q, u_{i}} I(u ; p, q)+\left[x_{k} \pm x_{i}\right] u_{j}^{-1} T_{q, u_{j}} I(u ; p, q)} \\
+\left[x_{i} \pm x_{j}\right] u_{k}^{-1} T_{q, u_{k}} I(u ; p, q)=0 .
\end{gathered}
$$

In view of this formula, we set

$$
J(x)=e(-Q(x)) I(u ; p, q), \quad Q(x)=\frac{1}{2 \delta}(x \mid x) .
$$

Note that

$$
Q(x+a \delta)=Q(x)+(a \mid x)+\frac{1}{2}(a \mid a) \delta
$$

for any $a \in P$. Since

$$
Q\left(x+v_{i} \delta\right)=Q(x)+\left(v_{i} \mid x\right)+\frac{1}{2} \delta=Q(x)+x_{i}+\frac{1}{2} \delta
$$

we have

$$
J\left(x+v_{i} \delta\right)=e(-Q(x)) q^{-\frac{1}{2}} u_{i}^{-1} T_{q, u_{i}} I(u ; p, q) \quad(i \in\{0,1, \ldots, 7\}) .
$$

Hence by (6.16) we obtain the three-term relations

$$
\left[x_{j} \pm x_{k}\right] J\left(x+v_{i} \delta\right)+\left[x_{k} \pm x_{i}\right] J\left(x+v_{j} \delta\right)+\left[x_{i} \pm x_{j}\right] J\left(x+v_{k} \delta\right)=0,
$$

namely

$$
\left[\left(v_{j} \pm v_{k} \mid x\right)\right] J\left(x+v_{i} \delta\right)+\left[\left(v_{k} \pm v_{i} \mid x\right)\right] J\left(x+v_{j} \delta\right)+\left[\left(v_{i} \pm v_{j} \mid x\right)\right] J\left(x+v_{k} \delta\right)=0
$$

for any triple $i, j, k \in\{0,1, \ldots, 7\}$.

On the basis of these observations, we construct a hypergeometric $\tau$-function on

$$
D_{\varpi}=\bigsqcup_{n \in \mathbb{Z}} H_{\varpi+n \delta} \subset V
$$

with the initial level $c=\varpi$. For this purpose we introduce the holomorphic function

$$
\mathcal{F}(x)=\prod_{0 \leq i<j \leq 7} \Gamma\left(u_{i} u_{j} ; p, q, q\right) \quad(x \in V) .
$$


Theorem 6.1 There exists a unique hypergeometric $\tau$-function $\tau(x)$ on $D_{\varpi}$ such that $\tau^{(n)}(x)=0(n<0)$ and

$$
\begin{aligned}
\tau^{(0)}(x) & =\mathcal{F}(x+\phi \delta)=\prod_{0 \leq i<j \leq 7} \Gamma\left(q u_{i} u_{j} ; p, q, q\right) \quad\left(x \in H_{\varpi}\right), \\
\tau^{(1)}(x) & =\mathcal{F}(x) J(x) \\
& =e(-Q(x)) I(u ; p, q) \prod_{0 \leq i<j \leq 7} \Gamma\left(u_{i} u_{j} ; p, q, q\right) \quad\left(x \in H_{\varpi+\delta}\right) .
\end{aligned}
$$

Furthermore $\tau(x)$ is a $W\left(E_{7}\right)$-invariant meromorphic function on $D_{\varpi}$.

Proof: We need to show that $\tau^{(0)}(x)$ and $\tau^{(1)}(x)$ satisfy the two conditions of Theorem 4.2. We first show that $\tau^{(0)}(x)\left(x \in H_{\varpi}\right)$ is $W\left(E_{7}\right)$-invariant. Since the $\mathfrak{S}_{8}$-invariance is manifest, we have only to show that it is invariant under the action of $s_{0}$. Noting that

$$
s_{0}\left(u_{i} u_{j}\right)= \begin{cases}p / u_{k} u_{l} & (\{i, j, k, l\}=\{0,1,2,3\}), \\ u_{i} u_{j} & (i \in\{0,1,2,3\}, j \in\{4,5,6,7\}), \\ p / u_{k} u_{l} & (\{i, j, k, l\}=\{4,5,6,7\})\end{cases}
$$

for $x \in H_{\varpi}$, we have

$$
s_{0}\left(\Gamma\left(q u_{i} u_{j} ; p, q, q\right)\right)=\Gamma\left(p q / u_{k} u_{l} ; p, q, q\right)=\Gamma\left(q u_{k} u_{l} ; p, q, q\right)
$$

for $\{i, j, k, l\}=\{0,1,2,3\}$ or $\{4,5,6,7\}$. Since $\Gamma\left(u_{i} u_{j} ; p, q, q\right)$ is $s_{0}$-invariant for $i \in$ $\{0,1,2,3\}, j \in\{4,5,6,7\}$, we have $s_{0}\left(\tau^{(0)}(x)\right)=\tau^{(0)}(x)$. We now verify that our $\tau^{(0)}(x)$ satisfy the condition (4.5) for any $C_{3}$-frame $\left\{ \pm a_{0}, \pm a_{1}, \pm a_{2}\right\}$ of type $\mathrm{II}_{1}$ as in (3.15). Since the $C_{3}$-frames of type $\mathrm{II}_{1}$ form a single $W\left(E_{7}\right)$-orbit, by the $W\left(E_{7}\right)$-invariance of $\tau^{(0)}(x)$ we may take

$$
\begin{aligned}
& a_{0}=\frac{1}{2}\left(v_{0}+v_{1}+v_{2}+v_{3}\right), \\
& a_{1}=\frac{1}{2}\left(v_{0}+v_{1}-v_{2}-v_{3}\right), \quad a_{2}=\frac{1}{2}\left(v_{0}-v_{1}+v_{2}-v_{3}\right)
\end{aligned}
$$

so that

$$
\left\{a_{0} \pm a_{1}\right\}=\left\{v_{0}+v_{1}, v_{2}+v_{3}\right\}, \quad\left\{a_{0} \pm a_{2}\right\}=\left\{v_{0}+v_{2}, v_{1}+v_{3}\right\}
$$

In this case one can directly check

$$
\frac{\tau^{(0)}\left(x \pm a_{1} \delta\right)}{\tau^{(0)}\left(x \pm a_{2} \delta\right)}=\frac{\theta\left(u_{0} u_{1} ; p\right) \theta\left(u_{2} u_{3} ; p\right)}{\theta\left(u_{0} u_{2} ; p\right) \theta\left(u_{1} u_{3} ; p\right)}=\frac{\left[x_{0}+x_{1}\right]\left[x_{2}+x_{3}\right]}{\left[x_{0}+x_{2}\right]\left[x_{1}+x_{3}\right]}=\frac{\left[\left(a_{0} \pm a_{1} \mid x\right)\right]}{\left[\left(a_{0} \pm a_{2} \mid x\right)\right]} .
$$

We next verify that $\tau^{(0)}(x)$ and $\tau^{(1)}(x)$ satisfy (4.6) for any $C_{3}$-frame $\left\{ \pm a_{0}, \pm a_{1}, \pm a_{2}\right\}$ with $\left(\phi \mid a_{i}\right)=\frac{1}{2}(i=0,1,2)$. Since $\tau^{(1)}(x)=e(-Q(x)) \Psi(u ; p, q, q)$ is $W\left(E_{7}\right)$-invariant, we have only to check (4.6) for $\left\{ \pm a_{0}, \pm a_{1}, \pm a_{2}\right\}=\left\{ \pm v_{0}, \pm v_{1}, \pm v_{2}\right\}$, namely,

$$
\begin{gathered}
{\left[x_{1} \pm x_{2}\right] \tau^{(0)}\left(x-v_{0} \delta\right) \tau^{(1)}\left(x+v_{0} \delta\right)+\left[x_{2} \pm x_{0}\right] \tau^{(0)}\left(x-v_{1} \delta\right) \tau^{(1)}\left(x+v_{1} \delta\right)} \\
+\left[x_{0} \pm x_{1}\right] \tau^{(0)}\left(x-v_{2} \delta\right) \tau^{(1)}\left(x+v_{2} \delta\right)=0
\end{gathered}
$$


Since

$$
\mathcal{F}\left(x+\phi \delta-v_{k} \delta\right) \mathcal{F}\left(x+v_{k} \delta\right)=\mathcal{F}(x+\phi \delta) \mathcal{F}(x)
$$

we have

$$
\begin{aligned}
\tau^{(0)}\left(x-v_{k} \delta\right) \tau^{(1)}\left(x+v_{k} \delta\right) & =\mathcal{F}\left(x+\phi \delta-v_{k} \delta\right) \mathcal{F}\left(x+v_{k} \delta\right) J\left(x+v_{k} \delta\right) \\
& =\mathcal{F}(x+\phi \delta) \mathcal{F}(x) J\left(x+v_{k} \delta\right)
\end{aligned}
$$

for each $k=0,1, \ldots, 7$. Hence the three-term relations (6.21) for $J(x)$ imply

$$
\begin{gathered}
{\left[x_{j} \pm x_{k}\right] \tau^{(0)}\left(x-v_{i} \delta\right) \tau^{(1)}\left(x+v_{i} \delta\right)+\left[x_{k} \pm x_{i}\right] \tau^{(0)}\left(x-v_{j} \delta\right) \tau^{(1)}\left(x+v_{j} \delta\right)} \\
+\left[x_{i} \pm x_{j}\right] \tau^{(0)}\left(x-v_{k} \delta\right) \tau^{(1)}\left(x+v_{j} \delta\right)=0
\end{gathered}
$$

for any tripe $i, j, k \in\{0,1, \ldots, 7\}$. The $W\left(E_{7}\right)$-invariance of $\tau(x)$ on $D_{\varpi}$ follows from the uniqueness of $\tau(x)$ and the $W\left(E_{7}\right)$-invariance of $\tau^{(0)}(x)$ and $\tau^{(1)}(x)$.

We next investigate the determinant formula of Theorem 4.3 for the hypergeometric $\tau$-function of Theorem 6.1 with initial condition

$$
\begin{aligned}
\tau^{(0)}(x) & =\prod_{0 \leq i<j \leq 7} \Gamma\left(q u_{i} u_{j} ; p, q, q\right) \quad\left(x \in H_{\varpi}\right), \\
\tau^{(1)}(x) & =e(-Q(x)) I(u ; p, q) \prod_{0 \leq i<j \leq 7} \Gamma\left(u_{i} u_{j} ; p, q, q\right) \quad\left(x \in H_{\varpi+\delta}\right) .
\end{aligned}
$$

For the recursive construction of $\tau^{(n)}(x)\left(x \in H_{\varpi+n \delta}\right)$ for $n=2,3, \ldots$, we use the $C_{8}$-frame $A_{1}=\left\{ \pm a_{0}, \pm a_{1}, \ldots, \pm a_{7}\right\}$ of type II of Example 1.2, where

$$
\begin{array}{ll}
a_{0}=\frac{1}{2}\left(v_{0}+v_{1}+v_{2}+v_{3}\right), & a_{4}=\frac{1}{2}\left(v_{4}-v_{5}-v_{6}+v_{7}\right), \\
a_{1}=\frac{1}{2}\left(v_{0}+v_{1}-v_{2}-v_{3}\right), & a_{5}=\frac{1}{2}\left(-v_{4}+v_{5}-v_{6}+v_{7}\right) . \\
a_{2}=\frac{1}{2}\left(v_{0}-v_{1}+v_{2}-v_{3}\right), & a_{6}=\frac{1}{2}\left(-v_{4}-v_{5}+v_{6}+v_{7}\right), \\
a_{3}=\frac{1}{2}\left(v_{0}-v_{1}-v_{2}+v_{3}\right), & a_{7}=\frac{1}{2}\left(v_{4}+v_{5}+v_{6}+v_{7}\right) .
\end{array}
$$

Note here that $\left(\phi \mid a_{0}\right)=\left(\phi \mid a_{7}\right)=1,\left(\phi \mid a_{i}\right)=0(i=1, \ldots, 6)$ and $a_{0}+a_{7}=\phi$. This $C_{8}$-frame $A_{1}$ contains the following $30 C_{3}$-frame of type $\mathrm{II}_{1}$ :

$$
\left\{ \pm a_{0}, \pm a_{i}, \pm a_{j}\right\}, \quad\left\{ \pm a_{7}, \pm a_{i}, \pm a_{j}\right\} \quad(1 \leq i<j \leq 6)
$$

Since

$$
\alpha_{0}=\phi-v_{0}-v_{1}-v_{2}-v_{3}=a_{7}-a_{0}
$$

we have

$$
s_{0}\left(a_{0}\right)=a_{7}, \quad s_{0}\left(a_{7}\right)=a_{0}, \quad s_{0}\left(a_{i}\right)=a_{i} \quad(i=1, \ldots, 6) .
$$

This means that the $C_{3}$-frames $\left\{ \pm a_{0}, \pm a_{i}, \pm a_{j}\right\}$ and $\left\{ \pm a_{7}, \pm a_{i}, \pm a_{j}\right\}$ are transformed to each other by $s_{0}$. To fix the idea, we consider below the cases of $C_{3}$-frames $\left\{ \pm a_{0}, \pm a_{1}, \pm a_{2}\right\}$ and $\left\{ \pm a_{7}, \pm a_{1}, \pm a_{2}\right\}$. 
Theorem 6.2 (Determinant formula) The $W\left(E_{7}\right)$-invariant hypergeometric $\tau$-function of Theorem 6.1 is expressed in terms of 2-directional Casorati determinants as

$$
\tau^{(n)}(x)=g^{(n)}(x) \operatorname{det}\left(\psi_{i j}^{(n)}(x)\right)_{i, j=1}^{n} \quad\left(x \in H_{\varpi+n \delta}\right)
$$

for $n=0,1,2, \ldots$, where the gauge factors $g^{(n)}(x)$ and the matrix elements $\psi_{i j}^{(n)}(x)$ are given as follows according to the $C_{3}$-frame of type $\mathrm{II}_{1}$ chosen for the recurrence.

(1) Case of the $C_{3}$-frame $\left\{ \pm a_{0}, \pm a_{1}, \pm a_{2}\right\}$ :

$$
\begin{aligned}
& g^{(n)}(x)=\frac{p^{\left(\begin{array}{l}
n \\
2
\end{array}\right) e(-n Q(x))}}{d^{(n)}(x)} \prod_{\substack{0 \leq i<j \leq 3 \\
\text { or } 4 \leq i<j \leq 7}} \Gamma\left(q u_{i} u_{j} ; p, q, q\right) \prod_{\substack{0 \leq i \leq 3 \\
4 \leq j \leq 7}} \Gamma\left(q^{1-n} u_{i} u_{j} ; p, q, q\right), \\
& \left.d^{(n)}(x)=q^{2\left(\begin{array}{c}
3 \\
2
\end{array}\right)}\left(p q / u_{0} u_{1}\right)\right)^{\left(\begin{array}{c}
n \\
2
\end{array}\right)} \prod_{k=1}^{n} \theta\left(q^{1-n} u_{0} u_{3} ; p ; q\right)_{k-1} \theta\left(q^{k-n} u_{0} / u_{3} ; p ; q\right)_{k-1}, \\
& \cdot \prod_{k=1}^{n} \theta\left(q^{1-n} u_{1} u_{2} ; p ; q\right)_{k-1} \theta\left(q^{k-n} u_{1} / u_{2} ; p ; q\right)_{k-1}, \\
& \psi_{i j}^{(n)}(x)=I\left(q^{n-i} t_{0}, q^{n-j} t_{1}, q^{j-1} t_{2}, q^{i-1} t_{3}, t_{4}, t_{5}, t_{6}, t_{7} ; p, q\right) \text {, } \\
& t_{k}= \begin{cases}u_{k} \sqrt{p q / u_{0} u_{1} u_{2} u_{3}} & (k=0,1,2,3), \\
u_{k} \sqrt{p q / u_{4} u_{5} u_{6} u_{7}} & (k=4,5,6,7) .\end{cases}
\end{aligned}
$$

(2) Case of the $C_{3}$-frame $\left\{ \pm a_{7}, \pm a_{1}, \pm a_{2}\right\}$ :

$$
\begin{aligned}
g^{(n)}(x)= & \frac{p^{\left(\begin{array}{c}
n \\
2
\end{array}\right) e(-n Q(x))}}{d^{(n)}(x)} \prod_{0 \leq i<j \leq 7} \Gamma\left(q^{1-n} u_{i} u_{j} ; p, q, q\right), \\
d^{(n)}(x)= & q^{-\left(\begin{array}{c}
n+1 \\
3
\end{array}\right)\left(u_{2} u_{3}\right)\left(\begin{array}{c}
n \\
2
\end{array}\right)} \prod_{k=1}^{n} \theta\left(q^{1-k} u_{0} u_{3} ; p ; q\right)_{k-1} \theta\left(q^{k-n} u_{0} / u_{3} ; p ; q\right)_{k-1} \\
& \cdot \prod_{k=1}^{n} \theta\left(q^{1-k} u_{1} u_{2} ; p ; q\right)_{k-1} \theta\left(q^{k-n} u_{1} / u_{2} ; p ; q\right)_{k-1}, \\
\psi_{i j}^{(n)}(x)= & I\left(q^{n-i} t_{0}, q^{n-j} t_{1}, q^{1-j} t_{2}, q^{1-i} t_{3}, t_{4}, t_{5}, t_{6}, t_{7} ; p, q\right), \quad t_{k}=q^{\frac{1}{2}(1-n)} u_{k} .
\end{aligned}
$$

Rewriting the 2-directional Casorati determinants above, we obtain expressions of the $W\left(E_{7}\right)$-invariant hypergeometric $\tau$-function in terms of multiple elliptic hypergeometric integrals. For the variables $t=\left(t_{0}, t_{1}, \ldots, t_{7}\right)$, we consider the multiple integrals as in Rains [12, 13]:

$$
\begin{aligned}
& I_{n}(t ; p, q)=I_{n}\left(t_{0}, t_{1}, \ldots, t_{7} ; p, q\right) \\
& =\frac{(p ; p)_{\infty}^{n}(q ; q)_{\infty}^{n}}{2^{n} n !(2 \pi \sqrt{-1})^{n}} \int_{C^{n}} \prod_{i=1}^{n} \frac{\prod_{k=0}^{7} \Gamma\left(t_{k} z_{i}^{ \pm 1} ; p, q\right)}{\Gamma\left(z_{i}^{ \pm 2} ; p, q\right)} \prod_{1 \leq i<j \leq n} \theta\left(z_{i}^{ \pm 1} z_{j}^{ \pm 1} ; p\right) \frac{d z_{1} \cdots d z_{n}}{z_{1} \cdots z_{n}} .
\end{aligned}
$$


We remark that $I_{n}(t ; p, q)$ is a special case (with $s=q$ ) of the $B C_{n}$ elliptic hypergeometric integral

$$
\frac{(p ; p)_{\infty}^{n}(q ; q)_{\infty}^{n}}{2^{n} n !(2 \pi \sqrt{-1})^{n}} \int_{C^{n}} \prod_{i=1}^{n} \frac{\prod_{k=0}^{7} \Gamma\left(t_{k} z_{i}^{ \pm 1} ; p, q\right)}{\Gamma\left(z_{i}^{ \pm 2} ; p, q\right)} \prod_{1 \leq i<j \leq n} \frac{\Gamma\left(s z_{i}^{ \pm 1} z_{j}^{ \pm 1} ; p, q\right)}{\Gamma\left(z_{i}^{ \pm 1} z_{j}^{ \pm 1} ; p, q\right)} \frac{d z_{1} \cdots d z_{n}}{z_{1} \cdots z_{n}}
$$

of type II.

Theorem 6.3 (Multiple integral representation) The $W\left(E_{7}\right)$-invariant hypergeometric $\tau$-function of Theorem 6.1 is expressed as follows in terms of multiple elliptic hypergeometric integrals:

$$
\begin{aligned}
& \tau^{(n)}(x)=p^{\left(\begin{array}{l}
n \\
2
\end{array}\right)} e(-n Q(x)) I_{n}\left(q^{\frac{1}{2}(1-n)} u ; p, q\right) \prod_{0 \leq i<j \leq 7} \Gamma\left(q^{1-n} u_{i} u_{j} ; p, q, q\right)
\end{aligned}
$$

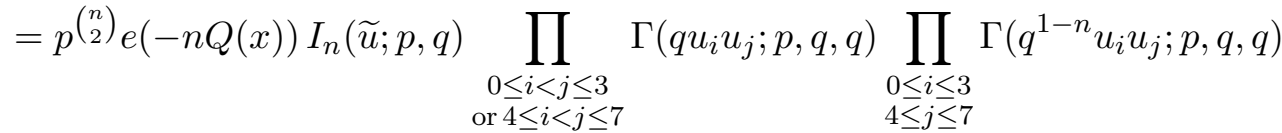

$$
\begin{aligned}
& \left(x \in H_{\varpi+n \delta}, n=0,1,2, \ldots\right),
\end{aligned}
$$

where

$$
\widetilde{u}_{k}= \begin{cases}u_{k} \sqrt{p q / u_{0} u_{1} u_{2} u_{3}} & (k=0,1,2,3) \\ u_{k} \sqrt{p q / u_{4} u_{5} u_{6} u_{7}} & (k=4,5,6,7)\end{cases}
$$

Theorems 6.2 and 6.3 will be proved in the next section.

We remark here that the equality of two expressions in (6.45) implies the transformation formula

$$
I_{n}(t ; p, q)=I_{n}(\widetilde{t} ; p, q) \prod_{0 \leq i<j \leq 3} \frac{\Gamma\left(q^{n} t_{i} t_{j} ; p, q, q\right)}{\Gamma\left(t_{i} t_{j} ; p, q, q\right)} \prod_{4 \leq i<j \leq 7} \frac{\Gamma\left(q^{n} t_{i} t_{j} ; p, q, q\right)}{\Gamma\left(t_{i} t_{j} ; p, q, q\right)}
$$

for the hypergeometric integral $I_{n}(t ; p, q)$ on $H_{\varpi+(2-n) \delta}\left(t_{0} t_{1} \cdots t_{7}=p^{2} q^{4-2 n}\right)$, where

$$
\widetilde{t}=\left(\widetilde{t_{0}}, \widetilde{t}_{1}, \ldots, \widetilde{t}_{7}\right), \quad \widetilde{t_{k}}=s_{0}\left(t_{k}\right)= \begin{cases}t_{k} \sqrt{p q^{2-n} / t_{0} t_{1} t_{2} t_{3}} & (k=0,1,2,3), \\ t_{k} \sqrt{p q^{2-n} / t_{4} t_{5} t_{6} t_{7}} & (k=4,5,6,7) .\end{cases}
$$

This is a special case of a transformation formula of Rains [13]. Note also, that the meromorphic function

$$
\Psi_{n}(t ; p, q)=I_{n}(t ; p, q) \prod_{0 \leq i<j \leq 7} \Gamma\left(t_{i} t_{j} ; p, q, q\right)
$$

on $H_{\varpi+(2-n) \delta}$ is $W\left(E_{7}\right)$-invariant. The invariance of $\Psi_{n}(t ; p, q)$ with respect to $w$ of (6.14) gives rise to the transformation formula

$$
\begin{aligned}
& I_{n}(t ; p, q)=I_{n}(\widehat{t} ; p, q) \prod_{0 \leq i<j \leq 7} \frac{\Gamma\left(q^{n} t_{i} t_{j} ; p, q, q\right)}{\Gamma\left(t_{i} t_{j} ; p, q, q\right)} \\
& \widehat{t}=\left(\widehat{t}_{0}, \widehat{t}_{1}, \ldots, \widehat{t}_{7}\right), \quad \widehat{t}_{k}=w\left(t_{k}\right)=\sqrt{p q^{2-n}} / t_{k} \quad(k=0,1, \ldots, 7)
\end{aligned}
$$


under the condition $t_{0} t_{1} \cdots t_{7}=p^{2} q^{4-2 n}$.

Applying this transformation formula, we obtain another expression of $\tau(x)$ of Theorem 6.3 .

$$
\begin{aligned}
\tau^{(n)}(x) & =p^{\left(\begin{array}{c}
n \\
2
\end{array}\right)} e(-n Q(x)) I_{n}\left(\sqrt{p q} u^{-1} ; p, q\right) \prod_{0 \leq i<j \leq 7} \Gamma\left(q u_{i} u_{j} ; p, q, q\right) \\
& =p^{\left(\begin{array}{c}
n \\
2
\end{array}\right)} e(-n Q(x)) I_{n}\left(\sqrt{p q} u^{-1} ; p, q\right) \prod_{0 \leq i<j \leq 7} \Gamma\left(p q / u_{i} u_{j} ; p, q, q\right) .
\end{aligned}
$$

In the notation of (6.49), the $W\left(E_{7}\right)$-invariant hypergeometric $\tau$-function is expressed as

$$
\begin{aligned}
\tau^{(n)}(x) & =p^{\left(\begin{array}{c}
n \\
2
\end{array}\right)} e(-n Q(x)) \Psi_{n}\left(q^{\frac{1}{2}(1-n)} u ; p, q\right) \\
& =p^{\left(\begin{array}{c}
n \\
2
\end{array}\right)} e(-n Q(x)) \Psi_{n}\left(p^{\frac{1}{2}} q^{\frac{1}{2}} u^{-1} ; p, q\right)
\end{aligned}
$$

for $x \in H_{\varpi+n \delta}(n=0,1,2, \ldots)$.

\section{Proof of Theorems 6.2 and 6.3}

In this section we prove Theorems 6.2 and 6.3 simultaneously.

We take the $C_{3}$-frame $\left\{ \pm a_{0}, \pm a_{1}, \pm a_{2}\right\}$ of type $\mathrm{II}_{1}$ as in (6.36) for constructing $\tau^{(n)}(x)$ $n=2,3, \ldots$. In this case, we have

$$
\begin{aligned}
& \left\{a_{0} \pm a_{1}\right\}=\left\{v_{0}+v_{1}, v_{2}+v_{3}\right\}, \quad\left\{a_{0} \pm a_{2}\right\}=\left\{v_{0}+v_{2}, v_{1}+v_{3}\right\} \\
& \left\{a_{1} \pm a_{2}\right\}=\left\{v_{0}-v_{3}, v_{1}-v_{2}\right\} .
\end{aligned}
$$

Hence, in order to apply Theorem 4.3, we need to decompose $\tau^{(1)}(x)$ into the product

$$
\tau^{(1)}(x)=g^{(1)}(x) \psi(x) \quad\left(x \in H_{\varpi+\delta}\right)
$$

with a gauge factor satisfying

$$
\frac{g^{(1)}\left(x \pm a_{1} \delta\right)}{g^{(1)}\left(x \pm a_{2} \delta\right)}=\frac{\left[\left(a_{0} \pm a_{1} \mid x\right)\right]}{\left[\left(a_{0} \pm a_{2} \mid x\right)\right]}=\frac{\theta\left(u_{0} u_{1} ; p\right) \theta\left(u_{2} u_{3} ; p\right)}{\theta\left(u_{0} u_{2} ; p\right) \theta\left(u_{1} u_{3} ; p\right)} \quad\left(x \in H_{\varpi+\delta}\right) .
$$

Then the gauge factors $g^{(n)}(x) n=2,3, \ldots$ are determined by the recurrence formula

$$
\frac{g^{(n-1)}\left(x-a_{0} \delta\right) g^{(n+1)}\left(x+a_{0} \delta\right)}{g^{(n)}\left(x \pm a_{1} \delta\right)}=\frac{\left[\left(a_{0} \pm a_{2} \mid x\right)\right]}{\left[\left(a_{1} \pm a_{2} \mid x\right)\right]}=\frac{\theta\left(u_{0} u_{2} ; p\right) \theta\left(u_{1} u_{3} ; p\right)}{u_{2} u_{3} \theta\left(u_{0} / u_{3} ; p\right) \theta\left(u_{1} / u_{2} ; p\right)}
$$

for $n=1,2, \ldots$ starting from $g^{(0)}(x)=\tau^{(0)}(x)$ and $g^{(1)}(x)$.

For this purpose, using the transformation formula (1) of Theorem 5.1, we rewrite $\tau^{(1)}(x)$ as

$$
\begin{aligned}
\tau^{(1)}(x) & =e(-Q(x)) I(\widetilde{u} ; p, q) \prod_{0 \leq i<j \leq 7} \Gamma\left(u_{i} u_{j} ; p, q, q\right) \prod_{\substack{0 \leq i<j \leq 3 \\
\text { or } 4 \leq i<j \leq 7}} \Gamma\left(u_{i} u_{j} ; p, q\right) \\
& =e(-Q(x)) I(\widetilde{u} ; p, q) \prod_{\substack{0 \leq i<j \leq 3 \\
\text { or } 4 \leq i<j \leq 7}} \Gamma\left(q u_{i} u_{j} ; p, q, q\right) \prod_{\substack{0 \leq i \leq 3 \\
4 \leq j \leq 7}} \Gamma\left(u_{i} u_{j} ; p, q, q\right),
\end{aligned}
$$


where

$$
\widetilde{u}=\left(\widetilde{u}_{0}, \widetilde{u}_{1}, \ldots, \widetilde{u}_{7}\right), \quad \widetilde{u}_{i}= \begin{cases}u_{i} \sqrt{p q / u_{0} u_{1} u_{2} u_{3}} & (i=0,1,2,3), \\ u_{i} \sqrt{p q / u_{4} u_{5} u_{6} u_{7}} & (i=4,5,6,7),\end{cases}
$$

and set

$$
\begin{aligned}
g^{(1)}(x) & =e(-Q(x)) \prod_{\substack{0 \leq i<j \leq 3 \\
\text { or } 4 \leq i<j \leq 7}} \Gamma\left(q u_{i} u_{j} ; p, q, q\right) \prod_{0 \leq i \leq 3,4 \leq j \leq 7} \Gamma\left(u_{i} u_{j} ; p, q\right), \\
\psi(x) & =I(\widetilde{u} ; p, q) \quad\left(x \in H_{\varpi+\delta}\right) .
\end{aligned}
$$

Then one can directly verify that $g^{(1)}(x)$ satisfies the condition (7.3). For the moment we set $t=\left(t_{0}, t_{1}, \ldots, t_{7}\right), t_{i}=\widetilde{u}_{i}(i=0,1, \ldots, 7)$, so that $\psi(x)=I(t ; p, q)$.

We now compute the determinant

$$
\begin{aligned}
& K^{(n)}(x)=\operatorname{det}\left(\psi_{i j}^{(n)}(x)\right)_{i, j=1}^{n}, \quad \psi_{i j}^{(n)}(x)=\psi\left(x+v_{i j}^{(n)} \delta\right), \\
& v_{i j}^{(n)}=(1-n) a_{0}+(n+1-i-j) a_{1}+(j-i) a_{2} \\
& \quad=(1-i, 1-j, j-n, i-n, 0,0,0,0) .
\end{aligned}
$$

Noting that the multiplicative coordinates of $x+v_{i j}^{(n)} \delta$ are given by

$$
\left(q^{1-i} u_{0}, q^{1-j} u_{1}, q^{j-n} u_{2}, q^{i-n} u_{3}, u_{4}, u_{5}, u_{6}\right),
$$

we obtain

$$
\psi_{i j}^{(n)}(x)=I\left(q^{n-i} t_{0}, q^{n-j} t_{1}, q^{j-1} t_{2}, q^{i-1} t_{3}, t_{4}, t_{5}, t_{6}, t_{7} ; p, q\right) .
$$

Hence $\psi_{i j}^{(n)}(x)$ is expressed as

$$
\begin{aligned}
\psi_{i j}^{(n)}(x) & =\kappa \int_{C} h(z) f_{i}(z) g_{j}(z) \frac{d z}{z}, \quad \kappa=\frac{(p ; p)_{\infty}(q ; q)_{\infty}}{4 \pi \sqrt{-1}}, \\
h(z) & =\frac{\prod_{k=0}^{7} \Gamma\left(t_{k} z^{ \pm 1} ; p, q\right)}{\Gamma\left(z^{ \pm 2} ; p, q\right)} \\
f_{i}(z) & =\theta\left(t_{0} z^{ \pm 1} ; p ; q\right)_{n-i} \theta\left(t_{3} z^{ \pm 1} ; p ; q\right)_{i-1}, \\
g_{j}(z) & =\theta\left(t_{1} z^{ \pm 1} ; p ; q\right)_{n-j} \theta\left(t_{2} z^{ \pm 1} ; p ; q\right)_{j-1},
\end{aligned}
$$

for $i, j=1,2, \ldots, n$, where $\theta(z ; p ; q)_{k}=\theta(z ; p) \theta(q z ; p) \cdots \theta\left(q^{k-1} z ; p\right)(k=0,1,2, \ldots)$. We rewrite the determinant $K^{(n)}(x)=\operatorname{det}\left(\psi_{i j}^{(n)}(x)\right)_{i, j=1}^{n}$ as

$$
\begin{aligned}
K^{(n)}(x) & =\frac{1}{n !} \sum_{\sigma, \tau \in \mathfrak{S}_{n}} \operatorname{sgn}(\sigma) \operatorname{sgn}(\tau) \prod_{k=1}^{n} \psi_{\sigma(k), \tau(k)}^{(n)}(x) \\
& =\frac{\kappa^{n}}{n !} \sum_{\sigma, \tau \in \mathfrak{S}_{n}} \operatorname{sgn}(\sigma) \operatorname{sgn}(\tau) \int_{C^{n}} \prod_{k=1}^{n} h\left(z_{k}\right) f_{\sigma(k)}\left(z_{k}\right) g_{\sigma(k)}\left(z_{k}\right) \frac{d z_{1} \cdots d z_{n}}{z_{1} \cdots z_{n}} \\
& =\frac{\kappa^{n}}{n !} \int_{C^{n}} h\left(z_{1}\right) \cdots h\left(z_{n}\right) \operatorname{det}\left(f_{i}\left(z_{j}\right)\right)_{i, j=1}^{n} \operatorname{det}\left(g_{i}\left(z_{j}\right)\right)_{i, j=1}^{n} \frac{d z_{1} \cdots d z_{n}}{z_{1} \cdots z_{n}} .
\end{aligned}
$$


Then the determinants $\operatorname{det}\left(f_{j}\left(z_{i}\right)\right)_{i, j=1}^{n}, \operatorname{det}\left(g_{j}\left(z_{i}\right)\right)_{i, j=1}^{n}$ can be evaluated by means of Warnaar's elliptic extension of the Krattenthaler determinant [18] (see also [9]).

Lemma 7.1 (Warnaar [18]) For a set of complex variables $\left(z_{1}, \ldots, z_{n}\right)$ and two parameters $a, b$, one has

$$
\begin{aligned}
& \operatorname{det}\left(\theta\left(a z_{i}^{ \pm 1} ; p ; q\right)_{j-1} \theta\left(b z_{i}^{ \pm 1} ; p ; q\right)_{n-j}\right)_{i, j=1}^{n} \\
& =q^{\left(\begin{array}{c}
n \\
3
\end{array}\right) a} a^{\left(\begin{array}{c}
n \\
2
\end{array}\right)} \prod_{k=1}^{n} \theta\left(b\left(q^{k-1} a\right)^{ \pm 1} ; p ; q\right)_{n-k} \prod_{1 \leq i<j \leq n} z_{i}^{-1} \theta\left(z_{i} z_{j}^{ \pm} ; p\right) .
\end{aligned}
$$

Sketch of proof: The left-hand side is invariant under the inversion $z_{i} \rightarrow z_{i}^{-1}$ for each $i=1, \ldots, n$, and alternating with respect to $\left(z_{1}, \ldots, z_{n}\right)$. Hence it is divisible by $\prod_{1 \leq i<j \leq n} z_{i}^{-1} \theta\left(z_{i} z_{j}^{ \pm} ; p\right)$. Also, the ratio of these two functions is elliptic with respect to the additive variable $\zeta_{i}$ with $z_{i}=e\left(\zeta_{i}\right)$ for each $i=1, \ldots, n$, and hence is constant. The constant on the right-hand side is determined by the substitution $z_{i}=q^{i-1} a(i=$ $1, \ldots, n)$ which makes the matrix on the left-hand side lower triangular.

We thus obtain

$$
K^{(n)}(x)=\operatorname{det}\left(\psi_{i j}^{(n)}(x)\right)_{i, j=1}^{n}=d^{(n)}(x) I_{n}(t ; p, q)
$$

where

$$
I_{n}(t ; p, q)=\frac{(p ; p)_{\infty}^{n}(q ; q)_{\infty}^{n}}{2^{n} n !(2 \pi \sqrt{-1})^{n}} \int_{C^{n}} \prod_{i=1}^{n} h\left(z_{i}\right) \prod_{1 \leq i<j \leq n} \theta\left(z_{i}^{ \pm 1} z_{j}^{ \pm 1} ; p\right) \frac{d z_{1} \cdots d z_{n}}{z_{1} \cdots z_{n}}
$$

and

$$
\begin{aligned}
& \left.d^{(n)}(x)=q^{2\left(\begin{array}{l}
n \\
3
\end{array}\right)\left(t_{2} t_{3}\right)}\right)^{\left(\begin{array}{c}
n \\
2
\end{array}\right)} \prod_{k=1}^{n} \theta\left(t_{0}\left(q^{k-1} t_{3}\right)^{ \pm 1} ; p ; q\right)_{n-k} \theta\left(t_{1}\left(q^{k-1} t_{2}\right)^{ \pm 1} ; p ; q\right)_{n-k} \\
& =q^{2\left(\begin{array}{c}
n \\
3
\end{array}\right)}\left(p q / u_{0} u_{1}\right)^{\left(\begin{array}{c}
n \\
2
\end{array}\right)} \prod_{(i, j)=(0,3),(1,2)} \prod_{k=1}^{n} \theta\left(q^{1-n} u_{i} u_{j} ; p ; q\right)_{k-1} \theta\left(q^{k-n} u_{i} / u_{k} ; p ; q\right)_{k-1} \text {. }
\end{aligned}
$$

Finally we determine the gauge factors $g^{(n)}(x) n=2,3, \ldots$ We set

$$
\mathcal{G}^{(n)}(x)=\prod_{\substack{0 \leq i<j \leq 3 \\ \text { or } 4 \leq i<j \leq 7}} \Gamma\left(q u_{i} u_{j} ; p, q, q\right) \prod_{\substack{0 \leq i \leq 3 \\ 4 \leq j \leq 7}} \Gamma\left(q^{1-n} u_{i} u_{j} ; p, q, q\right),
$$

so that $g^{(0)}(x)=\mathcal{G}^{(0)}(x), g^{(1)}(x)=e(-Q(x)) \mathcal{G}^{(1)}(x)$. By a direct computation, we see that $\mathcal{G}^{(n)}(x)$ satisfy the recurrence formula

$$
\frac{\mathcal{G}^{(n-1)}\left(x-a_{0} \delta\right) \mathcal{G}^{(n+1)}\left(x+a_{0} \delta\right)}{\mathcal{G}^{(n)}\left(x \pm a_{1} \delta\right)}=\theta\left(u_{0} u_{2} ; p\right) \theta\left(u_{0} u_{3} ; p\right) \theta\left(u_{1} u_{2} ; p\right) \theta\left(u_{1} u_{3} ; p\right) .
$$


If we set $g^{(n)}(x)=\mathcal{G}^{(n)}(x) c^{(n)}(x)$, the recurrence formula to be satisfied by $c^{(n)}(x)$ is given by

$$
\frac{c^{(n-1)}\left(x-a_{0} \delta\right) c^{(n+1)}\left(x+a_{0} \delta\right)}{c^{(n)}\left(x \pm a_{1} \delta\right)}=\frac{1}{u_{2} u_{3} \theta\left(u_{0} u_{3}^{ \pm 1} ; p\right) \theta\left(u_{1} u_{2}^{ \pm 1} ; p\right)}
$$

with the initial conditions $c^{(0)}(x)=1, c^{(1)}(x)=e(-Q(x))$. On the other hand, one can verify that the functions $d^{(n)}(x)$, which appeared in the evaluation of the determinant $K^{(n)}(x)$, satisfy

$$
\frac{d^{(n-1)}\left(x-a_{0} \delta\right) d^{(n+1)}\left(x+a_{0} \delta\right)}{d^{(n)}\left(x \pm a_{1} \delta\right)^{2}}=\left(p / u_{0} u_{1}\right) \theta\left(u_{0} u_{3}^{ \pm 1} ; p\right) \theta\left(u_{1} u_{2}^{ \pm 1} ; p\right) .
$$

If we set $c^{(n)}(x)=e^{(n)}(x) / d^{(n)}(x)$, the recurrence formula for $e^{(n)}(x)$ is determined as

$$
\frac{e^{(n-1)}\left(x-a_{0} \delta\right) e^{(n+1)}\left(x+a_{0} \delta\right)}{e^{(n)}\left(x \pm a_{1} \delta\right)}=p / u_{0} u_{1} u_{2} u_{3}=p e\left(-2\left(a_{0} \mid x\right)\right) .
$$

With the initial conditions $e^{(0)}(x)=1, e^{(1)}(x)=e(-Q(x))$, this recurrence is solved as

$$
e^{(n)}(x)=p^{\left(\begin{array}{c}
n \\
2
\end{array}\right)} e(-n Q(x)) \quad(n=0,1,2, \ldots) .
$$

Hence the gauge factors $g^{(n)}(x)$ are determined as

$$
g^{(n)}(x)=\frac{p^{\left(\begin{array}{c}
n \\
2
\end{array}\right) e(-n Q(x))}}{d^{(n)}(x)} \mathcal{G}^{(n)}(x) \quad(n=0,1,2, \ldots) .
$$

Since $K^{(n)}(x)=d^{(n)}(x) I_{n}(t ; p, q)$ with $t=\widetilde{u}$ as in (7.6), we also obtain

$$
\begin{aligned}
\tau^{(n)}(x) & =\frac{p^{\left(\begin{array}{c}
n \\
2
\end{array}\right)} e(-n Q(x))}{d^{(n)}(x)} \mathcal{G}^{(n)}(x) \operatorname{det}\left(\psi_{i j}^{(n)}(x)\right)_{i, j=1}^{n} \\
& =p^{\left(\begin{array}{c}
n \\
2
\end{array}\right)} e(-n Q(x)) \mathcal{G}^{(n)}(x) I_{n}(\widetilde{u} ; p, q) .
\end{aligned}
$$

This proves Theorem 6.2. (1) and the second equality of Theorem 6.3.

We already know by Theorem 6.1 that $\tau^{(n)}(x)\left(x \in H_{\varpi+n \delta}\right)$ are $W\left(E_{7}\right)$-invariant for all $n=0,1,2, \ldots$. Namely $w\left(\tau^{(n)}(x)\right)=\tau^{(n)}(x)$ for any $w \in W\left(E_{7}\right)$. This means that, for each $w \in W\left(E_{7}\right), \tau^{(n)}(x)\left(x \in H_{\varpi+n \delta}\right)$ has a determinant formula

$$
\tau^{(n)}(x)=\frac{p^{\left(\begin{array}{c}
n \\
2
\end{array}\right)} e(-n Q(x))}{\gamma^{(n)}(x)} \mathcal{F}^{(n)}(x) \operatorname{det}\left(\varphi_{i j}^{(n)}(x)\right)_{i, j=1}^{n}
$$

and a multiple integral representation

$$
\tau^{(n)}(x)=p^{\left(\begin{array}{l}
n \\
2
\end{array}\right)} e(-n Q(x)) \mathcal{F}^{(n)}(x) I_{n}(t ; p, q),
$$


where $\gamma^{(n)}, \mathcal{F}^{(n)}, \varphi_{i j}^{(n)}(i, j=1, \ldots, n)$ and $t_{k}(k=0,1, \ldots, 7)$ are specified by applying $w$ to the functions $d^{(n)}, \mathcal{G}^{(n)}, \psi_{i j}^{(n)}$ and $\widetilde{u}_{k}$ on $H_{\varpi+n \delta}$, respectively. When $w=s_{0}$, by the transformation

$$
s_{0}\left(u_{i}\right)= \begin{cases}u_{i} \sqrt{p q^{n} / u_{0} u_{1} u_{2} u_{3}} & (i=0,1,2,3), \\ u_{i} \sqrt{p q^{n} / u_{4} u_{5} u_{6} u_{7}} & (i=4,5,6,7),\end{cases}
$$

we obtain

$$
\begin{aligned}
& \gamma^{(n)}(x)=s_{0}\left(d^{(n)}(x)\right) \\
& =q^{2\left(\begin{array}{l}
n \\
3
\end{array}\right)}\left(q^{1-n} u_{2} u_{3}\right)^{\left(\begin{array}{c}
n \\
2
\end{array}\right)} \prod_{(i, j)=(0,3),(1,2)} \prod_{k=1}^{n} \theta\left(p q / u_{i} u_{j} ; p ; q\right)_{k-1} \theta\left(q^{k-n} u_{i} / u_{j} ; p ; q\right)_{k-1} \\
& =q^{-\left(\begin{array}{c}
n+1 \\
3
\end{array}\right)}\left(u_{2} u_{3}\right)^{\left(\begin{array}{c}
n \\
2
\end{array}\right)} \prod_{(i, j)=(0,3),(1,2)} \prod_{k=1}^{n} \theta\left(q^{1-k} u_{i} u_{j} ; p ; q\right)_{k-1} \theta\left(q^{k-n} u_{i} / u_{j} ; p ; q\right)_{k-1}
\end{aligned}
$$

and

$$
\begin{aligned}
\mathcal{F}^{(n)}(x) & =s_{0}\left(\mathcal{G}^{(n)}(x)\right)=\prod_{0 \leq i<j \leq n} \Gamma\left(q^{1-n} u_{i} u_{j} ; p, q\right), \\
\varphi_{i j}^{(n)}(x) & =s_{0}\left(\psi_{i j}^{(n)}(x)\right)=I\left(q^{n-i} t_{0}, q^{n-j} t_{1}, q^{j-1} t_{2}, q^{i-1} t_{3}, t_{4}, t_{5}, t_{6}, t_{7} ; p, q\right), \\
t_{k} & =q^{\frac{1}{2}(1-n)} u_{k} .
\end{aligned}
$$

Formulas (7.25) and (7.26) for this case $w=s_{0}$ give Theorem 6.2, (2) and the first equality of Theorem 6.3 .

\section{Transformation of hypergeometric $\tau$-functions}

From the $W\left(E_{7}\right)$-invariant hypergeometric $\tau$-function on $D_{\varpi}$ discussed above, one can construct a class of $\tau$-functions of hypergeometric type by the transformations in Theorem 2.3. In this section we give some remarks on this class of ORG $\tau$-functions.

In what follows, for a root $\alpha \in \Delta\left(E_{8}\right)$ and a constant $\kappa \in \mathbb{C}$, we denote by

$$
H_{\alpha, \kappa}=\{x \in V \mid(\alpha \mid x)=\kappa\}
$$

the hyperplane of level $\kappa$ with respect to $\alpha$. We consider a meromorphic function $\tau(x)$ on the disjoint union of parallel hyperplanes

$$
D_{\alpha, \kappa}=\bigsqcup_{n \in \mathbb{Z}} H_{\alpha, \kappa+n \delta} \subset V .
$$

Denoting by $\tau^{(n)}(x)$ the restriction of $\tau(x)$ to the $n$th hyperplane $H_{\kappa+n \delta}$, we say that an ORG $\tau$-function $\tau(x)$ on $D_{\alpha, \kappa}$ is a hypergeometric $\tau$-function of direction $\alpha$ with initial level $\kappa$ if $\tau^{(n)}(x)=0$ for $n<0$ and $\tau^{(0)}(x) \not \equiv 0$. 
In what follows, we use the notation

$$
\Psi_{n}(t ; p, q)=I_{n}(t ; p, q) \prod_{0 \leq i<j \leq 7} \Gamma\left(t_{i} t_{j} ; p, q, q\right) .
$$

As we have seen in (6.50), this function satisfies

$$
\Psi_{n}(t ; p, q)=\Psi_{n}\left(p^{\frac{1}{2}} q^{\frac{1}{2}(2-n)} t^{-1} ; p, q\right)
$$

under the balancing condition $t_{0} t_{1} \cdots t_{7}=p^{2} q^{4-2 n}$.

Theorem 8.1 With respect to the directions $\pm \phi$ and the initial levels $\pm \varpi(e(\varpi)=p)$, the following four functions are $W\left(E_{7}\right)$-invariant hypergeometric $\tau$-functions.

(0) $\tau_{++}(x)$ on $D_{\phi, \varpi}=\bigsqcup_{n \in \mathbb{Z}} H_{\phi, \varpi+n \delta}$ :

$\tau_{++}^{(n)}(x)=p^{\left(\begin{array}{c}n \\ 2\end{array}\right)} e(-n Q(x)) \Psi_{n}\left(q^{\frac{1}{2}(1-n)} u ; p, q\right)=p^{\left(\begin{array}{c}n \\ 2\end{array}\right)} e(-n Q(x)) \Psi_{n}\left(p^{\frac{1}{2}} q^{\frac{1}{2}} u^{-1} ; p, q\right)$.

(1) $\tau_{+-}(x)$ on $D_{\phi,-\varpi}=\bigsqcup_{n \in \mathbb{Z}} H_{\phi,-\varpi+n \delta}$ :

$$
\tau_{+-}^{(n)}(x)=\Psi_{n}\left(p^{\frac{1}{2}} q^{\frac{1}{2}(1-n)} u ; p, q\right)=\Psi_{n}\left(q^{\frac{1}{2}} u^{-1} ; p, q\right) .
$$

(2) $\tau_{-+}(x)$ on $D_{-\phi, \varpi}=\bigsqcup_{n \in \mathbb{Z}} H_{-\phi, \varpi+n \delta}=\bigsqcup_{n \in \mathbb{Z}} H_{\phi,-\varpi-n \delta}$ :

$$
\tau_{-+}^{(n)}(x)=p^{\left(\begin{array}{c}
n \\
2
\end{array}\right)} e(-n Q(x)) \Psi_{n}\left(p^{\frac{1}{2}} q^{\frac{1}{2}} u ; p, q\right)=p^{\left(\begin{array}{c}
n \\
2
\end{array}\right)} e(-n Q(x)) \Psi_{n}\left(q^{\frac{1}{2}(1-n)} u^{-1} ; p, q\right) .
$$

(3) $\tau_{--}(x)$ on $D_{-\phi,-\varpi}=\bigsqcup_{n \in \mathbb{Z}} H_{-\phi,-\varpi+n \delta}=\bigsqcup_{n \in \mathbb{Z}} H_{\phi, \varpi-n \delta}$ :

$$
\tau_{--}^{(n)}(x)=\Psi_{n}\left(q^{\frac{1}{2}} u ; p, q\right)=\Psi_{n}\left(p^{\frac{1}{2}} q^{\frac{1}{2}(1-n)} u^{-1} ; p, q\right) .
$$

Proof: The function $\tau_{++}(x)$ is the $W\left(E_{8}\right)$-invariant hypergeometric $\tau$-function of direction $\phi$ with initial level $\varpi$ discussed in previous sections. We apply the translation by $-\phi \varpi$ to $\tau(x)=\tau_{++}(x)$ as in Theorem 2.3, (3) to construct a $\tau$-function

$$
\widetilde{\tau}(x)=e(S(x ;-\phi, \varpi)) \tau(x+\phi \varpi)
$$

on $D_{\phi, \varpi}-\phi \varpi=D_{\phi,-\varpi}$. We look at the prefactor of $\widetilde{\tau}^{(n)}(x)\left(x \in H_{\phi,-\varpi+n \delta}\right)$ :

$$
e(S(x ;-\phi, \varpi)) p^{\left(\begin{array}{c}
n \\
2
\end{array}\right)} e(-n Q(x))=e(S(x ;-\phi, \varpi)) e\left(-n Q(x)+\left(\begin{array}{c}
n \\
2
\end{array}\right) \varpi\right) .
$$

Noting that $\eta_{\varpi}=-1$ in this case, we compute

$$
\begin{aligned}
S(x ;-\phi, \varpi) & =\frac{1}{2 \delta^{2}}(\phi \mid x)(x \mid x+\phi \varpi)=\frac{1}{2 \delta^{2}}(\phi \mid x)(x \mid x)+\frac{\varpi}{2 \delta^{2}}(\phi \mid x)^{2} \\
& =\frac{n}{2 \delta}(x \mid x)+\frac{n^{2}}{2} \varpi-\frac{\varpi}{2 \delta^{2}}(x \mid x)-\frac{n}{2} \frac{\varpi^{2}}{\delta}+\frac{\varpi^{3}}{2 \delta^{2}} .
\end{aligned}
$$

for $(\phi \mid x)=-\varpi+n \delta$. Combining this with

$$
-n Q(x+\phi \varpi)=-\frac{n}{2 \delta}(x+\phi \varpi \mid x+\phi \varpi)=-\frac{n}{2 \delta}(x \mid x)-n^{2} \varpi,
$$


we obtain

$$
\begin{aligned}
S(x ;-\phi, \varpi)-n Q(x)+\left(\begin{array}{c}
n \\
2
\end{array}\right) \varpi & =-\frac{\varpi}{2 \delta^{2}}(x \mid x)-n \frac{\varpi(\varpi+\delta)}{2 \delta}+\frac{\varpi^{3}}{2 \delta^{2}} \\
& =-\frac{\varpi}{2 \delta^{2}}(x \mid x)-\frac{\varpi(\varpi+\delta)}{2 \delta^{2}}(\phi \mid x)-\frac{\varpi^{2}}{2 \delta}
\end{aligned}
$$

by $n=((\phi \mid x)+\varpi) / \delta$. Hence the prefactor (8.10) for $\widetilde{\tau}^{(n)}(x)$ is determined as

$$
e\left(-\frac{\varpi}{2 \delta^{2}}(x \mid x)-\frac{\omega(\omega+\delta)}{2 \delta^{2}}(\phi \mid x)-\frac{\omega^{2}}{2 \delta}\right) .
$$

This factor does not effect on the Hirota equations, and can be eliminated by Theorem 2.3. (1). We thus obtain the $\tau$-function $\tau_{+-}(x)$ of (8.6) by replacing $e(x)=u$ in the last two factors of $\tau_{++}(x)$ with $e(x+\phi \omega)=p^{\phi} u=p^{\frac{1}{2}} u$. The other two functions $\tau_{-+}(x)$ and $\tau_{--}(x)$ are obtained by replacing $x$ in $\tau_{++}(x)$ and $\tau_{+-}(x)$ with $-x$, respectively.

We now apply Theorem [2.3, (3) for constructing hypergeometric $\tau$-functions with initial level 0 . Let $a \in P$ be a vector with $(\phi \mid a)=1$, so that

$$
D_{\phi,-\varpi}+a \varpi=D_{\phi, 0}=\bigsqcup_{n \in \mathbb{Z}} H_{\phi, n \delta} .
$$

Then, from

$$
\tau_{+-}^{(n)}(x)=\Psi_{n}\left(p^{\frac{1}{2}} q^{\frac{1}{2}(1-n)} u ; p, q\right)=\Psi_{n}\left(q^{\frac{1}{2}} u^{-1} ; p, q\right)
$$

we obtain a hypergeometric $\tau$-function

$$
\begin{aligned}
\tau_{a}(x) & =e(S(x ; a, \varpi)) \tau_{+-}(x-a \varpi) \\
& =e(S(x ; a, \varpi)) \Psi_{n}\left(p^{-a} p^{\frac{1}{2}} q^{\frac{1}{2}(1-n)} u ; p, q\right) \\
& =e(S(x ; a, \varpi)) \Psi_{n}\left(p^{a} q^{\frac{1}{2}} u^{-1} ; p, q\right)
\end{aligned}
$$

on $D_{\phi, 0}$, where

$$
S(x ; a, \varpi)=-\frac{1}{2 \delta^{2}}(a \mid x)(x \mid x-a \varpi) .
$$

When $a \in \Delta\left(E_{8}\right)$, namely $(a \mid a)=2$, there are 56 choices of $a$ with $(\phi \mid a)=1$ :

$$
a=v_{k}+v_{l}, \quad \phi-v_{k}-v_{l} \quad(0 \leq k<l \leq 7) .
$$

Those $\tau$-functions $\tau_{a}(x)$ on $D_{\phi, 0}$ correspond to the 56 hypergeometric $\tau$-functions in the trigonometric case studied by Masuda [8].

In general, let $\omega=k+l \varpi \in \Omega(k, l \in \mathbb{Z})$ a period, and take two vectors $a, b \in P$ with $(\phi \mid a)=k,(\phi \mid b)=l+1$. Then

$$
e(S(x ; b, \varpi)) \tau_{+-}(x-a-b \varpi)
$$

is a hypergeometric $\tau$-function on $D_{\phi, \omega}$. Furthermore, let $\alpha \in \Delta\left(E_{8}\right)$ be an arbitrary root of the root system of type $E_{8}$, and choose a $w \in W\left(E_{8}\right)$ such that $\alpha=w$. $\phi$. Then

$$
w\left(e(S(x ; b, \varpi)) \tau_{+-}(x-a-b \varpi)\right)=e\left(S\left(w^{-1} . x ; b, \varpi\right)\right) \tau_{+-}\left(w^{-1} . x-a-b \varpi\right)
$$

provides a hypergeometric $\tau$-function on $D_{\alpha, \omega}$ in the direction $\alpha$ with initial level $\omega$. 


\section{Relation to the framework of point configurations}

In this section, we give some remarks on how ORG $\tau$-functions are related to the notion of lattice $\tau$-functions associated with the configuration of generic nine points in $\mathbb{P}^{2}$.

\subsection{Realization of the affine root system $E_{8}^{(1)}$}

We first recall from [5] the realization of the affine root system of type $E_{8}^{(1)}$ in the context of the configuration of generic nine points in $\mathbb{P}^{2}$ (see also Dolgachev-Ortland [2]). We consider the 10-dimensional complex vector space

$$
\mathfrak{h}=\mathfrak{h}_{3,9}=\mathbb{C} e_{0} \oplus \mathbb{C} e_{1} \oplus \cdots \oplus \mathbb{C} e_{9}
$$

with basis $\left\{e_{0}, e_{1}, \ldots, e_{9}\right\}$, and define a scalar product (nondegenerate symmetric $\mathbb{C}$ bilinear form) $(\cdot \mid \cdot): \mathfrak{h} \times \mathfrak{h} \rightarrow \mathbb{C}$ by

$$
\begin{gathered}
\left(e_{0} \mid e_{0}\right)=-1, \quad\left(e_{j} \mid e_{j}\right)=1 \quad(j \in\{1, \ldots, 9\}), \\
\left(e_{i} \mid e_{j}\right)=0 \quad(i, j \in\{0,1, \ldots, 9\} ; i \neq j) .
\end{gathered}
$$

This vector space is regarded as the complexification of the lattice

$$
L=L_{3,9}=\mathbb{Z} e_{0} \oplus \mathbb{Z} e_{1} \oplus \mathbb{Z} e_{2} \oplus \cdots \oplus \mathbb{Z} e_{9} \subset \mathfrak{h}=\mathfrak{h}_{3,9}
$$

endowed with the symmetric $\mathbb{Z}$-bilinear form $(\cdot \mid \cdot): L \times L \rightarrow \mathbb{Z}$. In geometric terms, $L=L_{3,9}$ is the Picard lattice associated with the blowup of $\mathbb{P}^{2}$ at generic nine points $p_{1}, \ldots, p_{9}$. The vectors $e_{0}$ and $e_{1}, \ldots, e_{9}$ denote the class of lines in $\mathbb{P}^{2}$ and those of exceptional divisors corresponding to $p_{1}, \ldots, p_{9}$ respectively, and the scalar product $(\cdot \mid \cdot)$ on $L$ represents the intersection form of divisor classes multiplied by -1 .

The root lattice of type $E_{8}^{(1)}$ is realized as

$$
Q\left(E_{8}^{(1)}\right)=\mathbb{Z} \alpha_{0} \oplus \mathbb{Z} \alpha_{1} \oplus \cdots \oplus \mathbb{Z} \alpha_{8} \subset L,
$$

where the simple roots $\alpha_{0}, \alpha_{1}, \ldots, \alpha_{8} \in \mathfrak{h}$ are defined by

$$
\alpha_{0}=e_{0}-e_{1}-e_{2}-e_{3}, \quad \alpha_{j}=e_{j}-e_{j+1} \quad(j=1, \ldots, 8)
$$

with Dynkin diagram

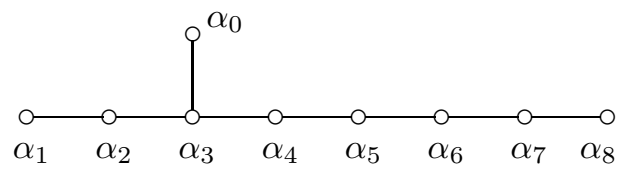

(These $\alpha_{j}$ are called the coroots $h_{j}$ in [5]). Note also that

$$
\begin{aligned}
c & =3 e_{0}-e_{1}-\cdots-e_{9} \\
& =3 \alpha_{0}+2 \alpha_{1}+4 \alpha_{2}+6 \alpha_{3}+5 \alpha_{4}+4 \alpha_{5}+3 \alpha_{6}+2 \alpha_{7}+\alpha_{8} \in Q\left(E_{8}^{(1)}\right)
\end{aligned}
$$


satisfies $\left(c \mid \alpha_{j}\right)=0$ for $j=0,1, \ldots, 8$. Denoting by $\mathfrak{h}^{*}=\operatorname{Hom}_{\mathbb{C}}(\mathfrak{h}, \mathbb{C})$ the dual space of $\mathfrak{h}$, we take the linear functions $\varepsilon_{j}=\left(e_{j} \mid \cdot\right) \in \mathfrak{h}^{*}(j=0,1, \ldots, 9)$ so that $\mathfrak{h}^{*}=$ $\mathbb{C} \varepsilon_{0} \oplus \mathbb{C} \varepsilon_{1} \oplus \cdots \oplus \mathbb{C} \varepsilon_{9}$, and regard $\varepsilon=\left(\varepsilon_{0} ; \varepsilon_{1}, \ldots, \varepsilon_{9}\right)$ as the canonical coordinates for $\mathfrak{h}$. We often identify $\mathfrak{h}^{*}$ with $\mathfrak{h}$ through the isomorphism $\nu: \mathfrak{h} \stackrel{\sim}{\rightarrow} \mathfrak{h}^{*}$ defined by $\nu(h)=(h \mid \cdot)$ $(h \in \mathfrak{h})$, and denote the induced scalar product by the same symbol $(\cdot \mid \cdot)$. When we regard the simple roots as $\mathbb{C}$-linear functions on $\mathfrak{h}$, they are expressed as $\nu\left(\alpha_{0}\right)=\varepsilon_{0}-\varepsilon_{1}-\varepsilon_{2}-\varepsilon_{3}$ and $\nu\left(\alpha_{j}\right)=\varepsilon_{j}-\varepsilon_{j+1}(j=1, \ldots, 8)$. Setting $\delta=(c \mid \cdot)=3 \varepsilon_{0}-\varepsilon_{1}-\cdots-\varepsilon_{9} \in \mathfrak{h}^{*}$, we regard below this null root $\delta \in \mathfrak{h}^{*}$ as the scaling unit for difference equations.

The root lattice of type $E_{8}$ is specified as $Q\left(E_{8}\right)=\mathbb{Z} \alpha_{0} \oplus \mathbb{Z} \alpha_{1} \oplus \cdots \oplus \mathbb{Z} \alpha_{7} \subset Q\left(E_{8}^{(1)}\right)$. The vector space $\mathfrak{h}$ is decomposed accordingly as

$$
\mathfrak{h}=\grave{\mathfrak{h}} \oplus \mathbb{C} c \oplus \mathbb{C} d, \quad \stackrel{\circ}{\mathfrak{h}}=\mathbb{C} \alpha_{0} \oplus \mathbb{C} \alpha_{1} \oplus \cdots \oplus \mathbb{C} \alpha_{7}, \quad d=-e_{9}-\frac{1}{2} c,
$$

where

$$
(c \mid h)=0, \quad(d \mid h)=0 \quad(h \in \mathfrak{h}) ; \quad(c \mid c)=0, \quad(c \mid d)=1, \quad(d \mid d)=0 .
$$

The 8-dimensional subspace $\stackrel{\circ}{\mathfrak{h}} \subset \mathfrak{h}$ can be identified with the vector space $V=\mathbb{C}^{8}$ that we have used throughout this paper for the realization of the root lattice $P=Q\left(E_{8}\right)$. Noting that $\stackrel{\circ}{\mathfrak{h}}=\{h \in \mathfrak{h} \mid(c \mid h)=0,(d \mid h)=0\}$ and $Q\left(E_{8}\right)=L \cap \grave{h}$, we define the orthonormal basis $\left\{v_{0}, v_{1}, \ldots, v_{7}\right\}$ for $\stackrel{\circ}{h}$ by

$$
v_{j}=e_{j}-\frac{1}{2}\left(e_{0}-e_{9}\right)+\frac{1}{2} c \quad(j=1, \ldots, 8), \quad v_{0}=-v_{8} .
$$

Then the highest root and the simple roots of type $E_{8}$ are expressed as

$$
\begin{aligned}
& \phi=\frac{1}{2}\left(v_{0}+v_{1}+\cdots+v_{7}\right)=c-\alpha_{8}, \\
& \alpha_{0}=\phi-v_{0}-v_{1}-v_{2}-v_{3}, \quad \alpha_{j}=v_{j}-v_{j+1} \quad(j=1, \ldots, 7)
\end{aligned}
$$

respectively, which recovers the situation of Section 1. In what follows, we identify $\mathfrak{h}$ with $V$ through this orthonormal basis $\left\{v_{0}, v_{1}, \ldots, v_{7}\right\}$. The corresponding $\mathbb{C}$-linear functions $x_{j}=\left(v_{j} \mid \cdot\right) \in \mathfrak{h}^{*}$ are realized as

$$
x_{j}=\varepsilon_{j}-\frac{1}{2}\left(\varepsilon_{0}-\varepsilon_{9}\right)+\frac{1}{2} \delta \quad(j=1, \ldots, 8), \quad x_{0}=-x_{8} .
$$

For each $\alpha \in \mathfrak{h}$ with $(\alpha \mid \alpha) \neq 0$, the reflection $r_{\alpha}: \mathfrak{h} \rightarrow \mathfrak{h}$ with respect to $\alpha$ is defined in the standard way by

$$
r_{\alpha}(h)=h-\left(\alpha^{\vee} \mid h\right) \alpha \quad(h \in \mathfrak{h}), \quad \alpha^{\vee}=2 \alpha /(\alpha \mid \alpha) .
$$

The affine Weyl group $W\left(E_{8}^{(1)}\right)=\left\langle s_{0}, s_{1}, \ldots, s_{8}\right\rangle$ of type $E_{8}^{(1)}$ (Coxeter group associated with diagram (9.6) ) then acts faithfully on $\mathfrak{h}$ through the simple reflections $s_{j}=r_{\alpha_{j}}(j=$ $0,1, \ldots 8)$. We remark that this group contains the symmetric group $\mathfrak{S}_{9}=\left\langle s_{1}, \ldots, s_{8}\right\rangle$ as a subgroup which permutes $e_{1}, \ldots, e_{9}$. The affine Weyl group $W\left(E_{8}^{(1)}\right)$ also acts on the dual space $\mathfrak{h}^{*}$ through $s_{j}=r_{\alpha_{j}}: \mathfrak{h}^{*} \rightarrow \mathfrak{h}^{*}$ defined in the same way as (9.13). These 
actions of $W\left(E_{8}^{(1)}\right)$ on $\mathfrak{h}$ and $\mathfrak{h}^{*}$ leave the two scalar products invariant. Note also that $c \in \mathfrak{h}$ and $\delta \in \mathfrak{h}^{*}$ are invariant under the action of $W\left(E_{8}^{(1)}\right)$.

Setting $\mathfrak{h}_{0}=\{\alpha \in \mathfrak{h} \mid(c \mid \alpha)=0\}$, for each $\alpha \in \mathfrak{h}_{0}$ we define the Kac translation ([3]) $T_{\alpha}: \mathfrak{h} \rightarrow \mathfrak{h}$ with respect to $\alpha$ by

$$
T_{\alpha}(h)=h+(c \mid h) \alpha-\left(\frac{1}{2}(\alpha \mid \alpha)(c \mid h)+(\alpha \mid h)\right) c \quad(h \in \mathfrak{h}) .
$$

It is directly verified that these $\mathbb{C}$-linear transformations $T_{\alpha} \in \operatorname{GL}(\mathfrak{h})\left(\alpha \in \mathfrak{h}_{0}\right)$ satisfy

$$
\begin{aligned}
& \text { (1) }\left(T_{\alpha}(h) \mid T_{\alpha}\left(h^{\prime}\right)\right)=\left(h \mid h^{\prime}\right) \quad\left(\alpha \in \mathfrak{h}_{0} ; h, h^{\prime} \in \mathfrak{h}\right), \\
& \text { (2) } T_{\alpha} T_{\beta}=T_{\beta} T_{\alpha}=T_{\alpha+\beta} \quad\left(\alpha, \beta \in \mathfrak{h}_{0}\right), \quad T_{k c}=\mathrm{id}_{\mathfrak{h}} \quad(k \in \mathbb{C}), \\
& \text { (3) } w T_{\alpha} w^{-1}=T_{w . \alpha} \quad\left(\alpha \in \mathfrak{h}_{0}, w \in W\left(E_{8}^{(1)}\right) .\right.
\end{aligned}
$$

Note also that

$$
T_{\alpha}(h)=h-(\alpha \mid h) c \quad(h \in \mathfrak{h},(c \mid h)=0), \quad T_{\alpha}(c)=c
$$

for any $\alpha \in \mathfrak{h}_{0}$. For a $\mathbb{Z}$-submodule $Q$ of $\mathfrak{h}_{0}$ given, we denote by $T(Q) \subset \operatorname{GL}(\mathfrak{h})$ the abelian subgroup of Kac translations $T_{\alpha}(\alpha \in Q)$. We remark that, if $\alpha \in \mathfrak{h}_{0}$ and $(\alpha \mid \alpha) \neq$ 0 , then the Kac translation $T_{\alpha}$ is expressed in the form $T_{\alpha}=r_{c-\alpha} \vee r_{\alpha} \vee$ as a product of two reflections. This implies in particular that the Kac translations $T_{\alpha_{j}}(j=0,1, \ldots, 8)$ by simple roots belong to the affine Weyl group $W\left(E_{8}^{(1)}\right)=\left\langle s_{0}, s_{1}, \ldots, s_{8}\right\rangle \subset \mathrm{GL}(\mathfrak{h})$. It is known $([3])$ in fact that $W\left(E_{8}^{(1)}\right)$ splits into the semidirect product

$$
W\left(E_{8}^{(1)}\right)=T\left(Q\left(E_{8}\right)\right) \rtimes W\left(E_{8}\right), \quad W\left(E_{8}\right)=\left\langle s_{0}, s_{1}, \ldots, s_{7}\right\rangle .
$$

We remark here that the linear action of $W\left(E_{8}^{(1)}\right)$ on $\mathfrak{h}$ extends to a bigger group $T\left(\mathfrak{h}_{0}\right) W\left(E_{8}^{(1)}\right)=T(V) \rtimes W\left(E_{8}\right)$ including the abelian group $T(V)$ of Kac translations with respect to $V=\mathbb{C} \otimes_{\mathbb{Z}} Q\left(E_{8}\right)$. Note that $T(V) \rtimes W\left(E_{8}\right)$ acts also on $\mathfrak{h}^{*}$ so that $\nu: \mathfrak{h} \stackrel{\sim}{\rightarrow} \mathfrak{h}^{*}$ intertwines its linear actions on $\mathfrak{h}$ and $\mathfrak{h}^{*}$.

Before proceeding further, we clarify how the linear actions of Kac translations on $\mathfrak{h}$ are related to the affine-linear actions of parallel translations on $V$. Note first that, for each $\kappa \in \mathbb{C}$, the hyperplane

$$
\mathfrak{h}_{\kappa}=\{h \in \mathfrak{h} \mid(c \mid h)=\kappa\} \subset \mathfrak{h}
$$

is stable by $W\left(E_{8}^{(1)}\right)$ and by $T\left(\mathfrak{h}_{0}\right)$. On this hyperplane $\mathfrak{h}_{\kappa}$ of level $\kappa$, the null root $\delta=(c \mid \cdot) \in \mathfrak{h}^{*}$ is identified with the constant function $\delta=\kappa$. For each $(\mu, \kappa) \in \mathbb{C} \times \mathbb{C}^{*}$, we define a quadratic mapping $\gamma_{(\mu, \kappa)}: V \rightarrow \mathfrak{h}_{\kappa}$ by

$$
\gamma_{(\mu, \kappa)}(x)=T_{\kappa^{-1} x}(\kappa d)-\mu c=x-\left(\frac{1}{2 \kappa}(x \mid x)+\mu\right) c+\kappa d \quad(x \in V) .
$$

These mappings $\gamma_{(\mu, \kappa)}$ induce the parametrization $\gamma_{\kappa}: V \times \mathbb{C} \stackrel{\sim}{\rightarrow} \mathfrak{h}_{\kappa}:(x ; \mu) \mapsto \gamma_{(\mu, \kappa)}(x)$ of $\mathfrak{h}_{\kappa}$ for each $\kappa \in \mathbb{C}^{*}$, as well as the isomorphism $\gamma: V \times \mathbb{C} \times \mathbb{C}^{*} \stackrel{\sim}{\rightarrow} \mathfrak{h} \backslash \mathfrak{h}_{0}$ of affine varieties such that

$$
\gamma(x ; \mu, \kappa)=x-\left(\frac{1}{2 \kappa}(x \mid x)+\mu\right) c+\kappa d \quad\left(x \in V, \mu \in \mathbb{C}, \kappa \in \mathbb{C}^{*}\right) .
$$


Furthermore, this isomorphism is equivariant with respect to the action of the group $T(V) \rtimes W\left(E_{8}\right)$ on $V \times \mathbb{C} \times \mathbb{C}^{*}$ specified by

$$
T_{v} w \cdot(x ; \mu, \kappa)=(w \cdot x+\kappa v ; \mu, \kappa) \quad\left(v \in V, w \in W\left(E_{8}\right)\right) .
$$

Through this isomorphism, the coordinates $(x ; \mu, \kappa)=\left(x_{0}, x_{1}, \ldots, x_{7} ; \mu, \kappa\right)$ for $V \times \mathbb{C} \times \mathbb{C}^{*}$ and $\varepsilon=\left(\varepsilon_{0} ; \varepsilon_{1}, \ldots, \varepsilon_{9}\right)$ for $\mathfrak{h} \backslash \mathfrak{h}_{0}$ are transformed into each other through

$$
\begin{aligned}
& x_{j}=\varepsilon_{j}-\frac{1}{2}\left(\varepsilon_{0}-\varepsilon_{9}\right)+\frac{1}{2} \delta \quad(j=1, \ldots, 8), \quad x_{0}=-x_{8}, \\
& \mu=-\frac{1}{2 \delta}(\varepsilon \mid \varepsilon), \quad \kappa=\delta
\end{aligned}
$$

where $(\varepsilon \mid \varepsilon)=-\varepsilon_{0}^{2}+\varepsilon_{1}^{2}+\cdots+\varepsilon_{9}^{2}$, and by

$$
\begin{aligned}
& \varepsilon_{0}=2 x_{0}-2(\phi \mid x)+3\left(\frac{1}{2 \kappa}(x \mid x)+\mu+\frac{1}{2} \kappa\right), \\
& \varepsilon_{j}=x_{j}+x_{0}-(\phi \mid x)+\frac{1}{2 \kappa}(x \mid x)+\mu+\frac{1}{2} \kappa \quad(j=1, \ldots, 7), \\
& \varepsilon_{8}=-(\phi \mid x)+\frac{1}{2 \kappa}(x \mid x)+\mu+\frac{1}{2} \kappa, \quad \varepsilon_{9}=\frac{1}{2 \kappa}(x \mid x)+\mu-\frac{1}{2} \kappa,
\end{aligned}
$$

where $(\phi \mid x)=\frac{1}{2}\left(x_{0}+x_{1}+\cdots+x_{7}\right),(x \mid x)=x_{0}^{2}+x_{1}^{2}+\cdots+x_{7}^{2}$.

\subsection{Lattice $\tau$-functions vs. ORG $\tau$-functions}

We now consider the $W\left(E_{8}^{(1)}\right)$-orbit

$$
M=M_{3,9}=W\left(E_{8}^{(1)}\right)\left\{e_{1}, \ldots, e_{9}\right\}=W\left(E_{8}^{(1)}\right) e_{9} \subset L=L_{3,9}
$$

in the Picard lattice. Noting that $e_{9} \in M$ is $W\left(E_{8}\right)$-invariant, we see that the natural mapping $W\left(E_{8}^{(1)}\right) \rightarrow M: w \mapsto w \cdot e_{9}$ induces the bijection $Q\left(E_{8}\right) \stackrel{\sim}{\rightarrow} M: \alpha \mapsto T_{\alpha} . e_{9}$. The orbit $M=W\left(E_{8}^{(1)}\right) e_{9}$ is intrinsically characterized as

$$
M=\{\Lambda \in L \mid(\Lambda \mid \Lambda)=1,(c \mid \Lambda)=-1\} .
$$

To see this, suppose that $\Lambda \in L$ satisfies $(\Lambda \mid \Lambda)=1$ and $(c \mid \Lambda)=-1$. Then, the difference $\beta=\Lambda-e_{9}$ satisfies $(\beta \mid \beta)+2\left(e_{9} \mid \beta\right)=0$ and $(c \mid \beta)=0$. This implies $\beta=\Lambda-e_{9} \in Q\left(E_{8}^{(1)}\right)$ and $T_{\beta} \cdot e_{9}=e_{9}+\beta=\Lambda$. Taking $\alpha=\beta+\left(e_{9} \mid \beta\right) c \in Q\left(E_{8}\right)$, we see that $\Lambda$ is uniquely expressed in the form

$$
\Lambda=e_{9}+\alpha+\frac{1}{2}(\alpha \mid \alpha) c, \quad \alpha \in Q\left(E_{8}\right),
$$

and hence $\Lambda=T_{\Lambda-e_{9}}^{-1} \cdot e_{9}=T_{\alpha}^{-1} \cdot e_{9}$. We remark that $\alpha$ is the unique element in $Q\left(E_{8}\right)$ such that $\Lambda-e_{9} \equiv \alpha(\bmod \mathbb{C} c)$, which we call the classical part of $\Lambda-e_{9} \in \mathfrak{h}_{0}$.

In [5], a system of lattice $\tau$-functions associated with the configurations of generic nine points in $\mathbb{P}^{2}$ is defined as a family of dependent variables $\tau_{\Lambda}$ indexed by $\Lambda \in M$ which admit an action of the affine Weyl group $W\left(E_{8}^{(1)}\right)$ such that

$$
w \cdot \tau_{\Lambda}=\tau_{w \cdot \Lambda} \quad\left(\Lambda \in M, w \in W\left(E_{8}^{(1)}\right)\right)
$$


and satisfy the quadratic relations

$$
\left[\varepsilon_{j k}\right]\left[\varepsilon_{j k l}\right] \tau_{e_{i}} \tau_{e_{0}-e_{i}-e_{l}}+\left[\varepsilon_{k i}\right]\left[\varepsilon_{k i l}\right] \tau_{e_{j}} \tau_{e_{0}-e_{j}-e_{l}}+\left[\varepsilon_{i j}\right]\left[\varepsilon_{i j l}\right] \tau_{e_{k}} \tau_{e_{0}-e_{k}-e_{l}}=0
$$

for all quadruples of mutually distinct $i, j, k, l \in\{1, \ldots, 9\}$, where $\varepsilon_{i j}=\varepsilon_{i}-\varepsilon_{j}$ and $\varepsilon_{i j k}=\varepsilon_{0}-\varepsilon_{i}-\varepsilon_{j}-\varepsilon_{k}$. (Here we use the notation $\tau_{\Lambda}$ instead of $\tau(\Lambda)$ as in [5] to make clear that $\Lambda$ is not an independent variable, but an index.) Under the condition (9.27), $\tau_{e_{9}}$ is $W\left(E_{8}\right)$-invariant, and all the functions $\tau_{\Lambda}(\Lambda \in M)$ are expressed as $\tau_{\Lambda}=T_{\Lambda-e_{9}}^{-1} \cdot \tau$ in terms of a single $W\left(E_{8}\right)$-invariant function $\tau=\tau_{e_{9}}$. We also remark that, if equation (9.28) holds for some quadruple of distinct $i, j, k, l \in\{1, \ldots, 9\}$, then it holds for all quadruples as a result of the action of $\mathfrak{S}_{9} \subset W\left(E_{8}^{(1)}\right)$. In the following, we use the notation $\sigma_{h}=[\nu(h)]=[(h \mid \cdot)]$ for $h \in \mathfrak{h}$, so that $\sigma_{e_{i j}}=\left[\varepsilon_{i j}\right]$ and $\sigma_{e_{i j k}}=\left[\varepsilon_{i j k}\right]$ where $e_{i j}=e_{i}-e_{j}$ and $e_{i j k}=e_{0}-e_{i}-e_{j}-e_{k}$. In this notation, equation (9.28) is rewritten as

$$
\sigma_{e_{j k}} \sigma_{e_{j k l}} \tau_{e_{i}} \tau_{e_{0}-e_{i}-e_{l}}+\sigma_{e_{k i}} \sigma_{e_{k i l}} \tau_{e_{j}} \tau_{e_{0}-e_{j}-e_{l}}+\sigma_{e_{i j}} \sigma_{e_{i j l}} \tau_{e_{k}} \tau_{e_{0}-e_{k}-e_{l}}=0 .
$$

As we will see below, equations (9.28) can be rewritten in a $W\left(E_{8}^{(1)}\right)$-invariant form.

To clarify the situation, let $X$ be a left $W\left(E_{8}^{(1)}\right)$-set. Noting that functions of the form $\sigma_{\alpha}=[(\alpha \mid \cdot)]\left(\alpha \in Q\left(E_{8}^{(1)}\right)\right)$ are defined over $\overline{\mathfrak{h}}=\mathfrak{h} / \mathbb{C} c$, we suppose that a $W\left(E_{8}^{(1)}\right)$ equivariant mapping $\gamma: X \rightarrow \overline{\mathfrak{h}}$ is given. Regarding those $\sigma_{\alpha}$ as functions defined on $X$ through $\gamma: X \rightarrow \overline{\mathfrak{h}}$, we can consider systems of lattice $\tau$-functions $\tau_{\Lambda}(\Lambda \in M)$ defined on $X$. In order to compare this notion with that of ORG $\tau$-functions on $X$, we assume that the extension $T\left(\frac{1}{2} Q\left(E_{8}\right)\right) \rtimes W\left(E_{8}\right)$ of $W\left(E_{8}^{(1)}\right)$ acts on $X$ so that $\gamma$ is an equivariant mapping. For a function $\varphi$ defined on a subset $U \subseteq X$, we define the action of $w \in T\left(\frac{1}{2} Q\left(E_{8}\right)\right) \rtimes W\left(E_{8}\right)$ on $\varphi$ to be the function $w \cdot \varphi$ on $w \cdot U$ such that $(w \cdot \varphi)(x)=\varphi\left(w^{-1} \cdot x\right)(x \in w \cdot U)$.

Proposition 9.1 Let $\tau$ be a $W\left(E_{8}\right)$-invariant function on $X$ and set $\tau_{\Lambda}=T_{\Lambda-e_{9}}^{-1} \cdot \tau$ for each $\Lambda \in M$. Then the following three conditions are equivalent:

(a) The equation

$$
\sigma_{e_{j k}} \sigma_{e_{j k l}} \tau_{e_{i}} \tau_{e_{0}-e_{i}-e_{l}}+\sigma_{e_{k i}} \sigma_{e_{k i l}} \tau_{e_{j}} \tau_{e_{0}-e_{j}-e_{l}}+\sigma_{e_{i j}} \sigma_{e_{i j l}} \tau_{e_{k}} \tau_{e_{0}-e_{k}-e_{l}}=0
$$

holds for each quadruple of mutually distinct $i, j, k, l \in\{1, \ldots, 9\}$.

(b) The equation

$$
\sigma_{u_{1}-u_{2}} \sigma_{u_{1}-u_{2}^{\prime}} \tau_{u_{0}} \tau_{u_{0}^{\prime}}+\sigma_{u_{2}-u_{0}} \sigma_{u_{2}-u_{0}^{\prime}} \tau_{u_{1}} \tau_{u_{1}^{\prime}}+\sigma_{u_{0}-u_{1}} \sigma_{u_{0}-u_{1}^{\prime}} \tau_{u_{2}} \tau_{u_{2}^{\prime}}=0
$$

holds for each sextuple of points $u_{i}, u_{i}^{\prime} \in M(i=0,1,2)$ such that

$$
u_{0}+u_{0}^{\prime}=u_{1}+u_{1}^{\prime}=u_{2}+u_{2}^{\prime}, \quad\left(u_{i}-u_{i}^{\prime} \mid u_{j}-u_{j}^{\prime}\right)=4 \delta_{i j} \quad(i, j=0,1,2) .
$$

(c) The equation

$$
\sigma_{a_{1} \pm a_{2}} T_{a_{0}} \cdot \tau T_{a_{0}}^{-1} \cdot \tau+\sigma_{a_{2} \pm a_{0}} T_{a_{1}} \cdot \tau T_{a_{1}}^{-1} \cdot \tau+\sigma_{a_{0} \pm a_{1}} T_{a_{2}} \cdot \tau T_{a_{2}}^{-1} \cdot \tau=0
$$

holds for each triple of vectors $a_{0}, a_{1}, a_{2} \in \frac{1}{2} Q\left(E_{8}\right)$ such that

$$
\left(a_{i} \mid a_{j}\right)=\delta_{i, j}, \quad \pm a_{i} \pm a_{j} \in Q\left(E_{8}\right) \quad(i, j=0,1,2) .
$$


Proof: Note first that equation (9.30) is a special case of (9.31) where $u_{0}=e_{i}, u_{1}=$ $e_{j}, u_{2}=e_{k}$ and $v=e_{0}-e_{l}$. Hence condition (b) implies (a). We consider equation (9.31) for a sextuple $u_{i}, u_{i}^{\prime} \in M(i=0,1,2)$ as in (b). Introducing

$$
\begin{aligned}
& g=\frac{1}{2}\left(u_{i}+u_{i}^{\prime}\right), \quad b_{i}=\frac{1}{2}\left(u_{i}-u_{i}^{\prime}\right) \quad(i=0,1,2), \\
& u_{i}=g+b_{i}, \quad u_{i}^{\prime}=g-b_{i} \quad(i=0,1,2),
\end{aligned}
$$

we rewrite equation (9.31) into the equation

$$
\sigma_{b_{1} \pm b_{2}} \tau_{g+b_{0}} \tau_{g-b_{0}}+\sigma_{b_{2} \pm b_{0}} \tau_{g+b_{1}} \tau_{g-b_{1}}+\sigma_{b_{0} \pm b_{1}} \tau_{g+b_{2}} \tau_{g-b_{2}}=0
$$

for a sextuple of points $g \pm b_{i} \in M(i=0,1,2)$ such that

$$
\left(b_{i} \mid b_{j}\right)=\delta_{i, j}, \quad \pm b_{i} \pm b_{j} \in Q\left(E_{8}^{(1)}\right) \quad(i, j=0,1,2) .
$$

In this setting, $b_{0}, b_{1}, b_{2}$ and $g$ are characterized by the conditions

$$
\begin{aligned}
& g \in \frac{1}{2} L, \quad(g \mid g)=0,(c \mid g)=-1 \\
& b_{i} \in \frac{1}{2} L, \quad\left(c \mid b_{i}\right)=0, \quad\left(g \mid b_{i}\right)=0, \quad\left(b_{i} \mid b_{j}\right)=\delta_{i j}, \quad \pm b_{i} \pm b_{j} \in Q\left(E_{8}^{(1)}\right) .
\end{aligned}
$$

Since $b_{i} \in \frac{1}{2} Q\left(E_{8}^{(1)}\right)(i=0,1,2)$, they are expressed as

$$
b_{i}=a_{i}+k_{i} c, \quad k_{i}=\left(g \mid a_{i}\right) \in \frac{1}{2} \mathbb{Z} \quad(i=0,1,2),
$$

where

$$
a_{i} \in \frac{1}{2} Q\left(E_{8}\right), \quad\left(g \mid a_{i}\right) \in \frac{1}{2} \mathbb{Z}, \quad\left(a_{i} \mid a_{j}\right)=\delta_{i j}, \quad \pm a_{i} \pm a_{j} \in Q\left(E_{8}\right) .
$$

Note that $\left(c \mid g-e_{9}\right)=0$ and hence $g-e_{9} \in \frac{1}{2} Q\left(E_{8}^{(1)}\right)$. In this situation we have

$$
T_{g-e_{9}}^{-1} \cdot a_{i}=a_{i}+\left(g-e_{9} \mid a_{i}\right) c=a_{i}+k_{i} c=b_{i} \quad(i=0,1,2),
$$

and hence we see that equations (9.36) and (9.33) are transformed into each other by the actions of $T_{g-e_{9}}$ and $T_{g-e_{9}}^{-1}$. These arguments show that condition (c) implies (b). Note also that equation (9.33) is a special case of (9.36) where $g=e_{9}+\frac{1}{2} c$ and $b_{i}=a_{i}$ $(i=0,1,2)$. We finally show that (a) implies (c). Suppose that equation (9.30) holds for some quadruple of distinct $i, j, k, l \in\{1, \ldots, 9\}$. Since it is a special case of (9.31), we see that equation (9.33) holds for some triple $a_{0}, a_{1}, a_{2} \in \frac{1}{2} Q\left(E_{8}\right)$ satisfying (9.34), namely for some $C_{3}$-frame $\left\{ \pm a_{0}, \pm a_{1}, \pm a_{2}\right\}$ in the terminology of Section 1. Since the Weyl group $W\left(E_{8}\right)$ acts transitively on the set of all $C_{3}$-frames, we see that equation (9.33) holds for all $C_{3}$-frames, which implies (c).

By abuse of terminology, we say that a function $\tau$ is an ORG $\tau$-function if it satisfies the non-autonomous Hirota equations (9.33) for all $C_{3}$-frames relative to $Q\left(E_{8}\right)$. Proposition 9.1 means that a family of functions $\tau_{\Lambda}(\Lambda \in M)$ on $X$ is a system of lattice $\tau$-functions if and only if $\tau=\tau_{e_{9}}$ is a $W\left(E_{8}\right)$-invariant ORG $\tau$-function on $X$. General ORG $\tau$-functions which are not necessarily $W\left(E_{8}\right)$-invariant can be interpreted as the system of lattice $\tau$-functions over a covering space of $X$. 
Setting

$$
\widetilde{X}=W\left(E_{8}\right) \times X=\left\{(w, x) \mid w \in W\left(E_{8}\right), x \in X\right\},
$$

we define an action of $T\left(\frac{1}{2} Q\left(E_{8}\right)\right) \rtimes W\left(E_{8}\right)$ on $\tilde{X}$ by

$$
T_{\alpha} w \cdot\left(w^{\prime}, x\right)=\left(w w^{\prime}, T_{\alpha} w \cdot x\right) \quad\left(\alpha \in \frac{1}{2} Q\left(E_{8}\right), w \in W\left(E_{8}\right)\right)
$$

so that the projection $\widetilde{X} \rightarrow X$ is equivariant. Let $U$ be a subset of $X$ and suppose that $U$ is stable by the group $T\left(Q\left(E_{8}\right)\right)$ of translations. Then the subset $\widetilde{U} \subseteq \widetilde{X}$ defined as

$$
\widetilde{U}=\left\{(w, x) \in \widetilde{X} \mid w \in W\left(E_{8}\right), x \in w \cdot U\right\}=\bigsqcup_{w \in W\left(E_{8}\right)}\{w\} \times w \cdot U
$$

is stable by the action of $W\left(E_{8}^{(1)}\right)=T\left(Q\left(E_{8}\right)\right) \rtimes W\left(E_{8}\right)$. To a function $\tau$ defined on $U$, we associate a function $\widetilde{\tau}$ on $\widetilde{U}$ by setting

$$
\widetilde{\tau}(w, x)=(w \cdot \tau)(x)=\tau\left(w^{-1} \cdot x\right) \quad\left(w \in W\left(E_{8}\right), x \in w \cdot U\right) .
$$

The function $\widetilde{\tau}$ on $\widetilde{U}$ is $W\left(E_{8}\right)$-invariant and $\tau$ on $U$ is recovered from $\widetilde{\tau}$ as $\widetilde{\tau}(1, x)=\tau(x)$ $(x \in U)$. Also, any $W\left(E_{8}\right)$-invariant function on $\widetilde{U}$ is obtained in this way from a function on $U$. Note also that the function $\tau$ on $U$ satisfies equations

$$
\sigma_{a_{1} \pm a_{2}} T_{a_{0}} \cdot \tau T_{a_{0}}^{-1} \cdot \tau+\sigma_{a_{2} \pm a_{0}} T_{a_{1}} \cdot \tau T_{a_{1}}^{-1} \cdot \tau+\sigma_{a_{0} \pm a_{1}} T_{a_{2}} \cdot \tau T_{a_{2}}^{-1} \cdot \tau=0
$$

for all $C_{3}$-frames $\left\{ \pm a_{0}, \pm a_{1}, \pm a_{2}\right\}$ relative to $Q\left(E_{8}\right)$ if and only if the $W\left(E_{8}\right)$-invariant function $\widetilde{\tau}$ on $\widetilde{U}$ satisfies

$$
\sigma_{a_{1} \pm a_{2}} T_{a_{0}} \cdot \widetilde{\tau} T_{a_{0}}^{-1} \cdot \widetilde{\tau}+\sigma_{a_{2} \pm a_{0}} T_{a_{1}} \cdot \widetilde{\tau} T_{a_{1}}^{-1} \cdot \widetilde{\tau}+\sigma_{a_{0} \pm a_{1}} T_{a_{2}} \cdot \widetilde{\tau} T_{a_{2}}^{-1} \cdot \widetilde{\tau}=0
$$

for all $C_{3}$-frames $\left\{ \pm a_{0}, \pm a_{1}, \pm a_{2}\right\}$ relative to $Q\left(E_{8}\right)$. Applying Proposition 9.1 to the mapping

$$
\widetilde{\gamma}: \widetilde{U} \rightarrow \overline{\mathfrak{h}}: \quad \widetilde{\gamma}(w, x)=\gamma(x) \quad\left(w \in W\left(E_{8}\right), x \in w \cdot U\right),
$$

and $\widetilde{\tau}$ on $\widetilde{U}$, we obtain the following characterization of an ORG $\tau$-function on $U$.

Proposition 9.2 For a function $\tau$ on a subset $U \subseteq X$, consider the function $\widetilde{\tau}$ defined on $\widetilde{U}$. Then $\tau$ is an ORG $\tau$-function on $U$ if and only if the functions $\widetilde{\tau}_{\Lambda}=T_{\Lambda-e_{9}}^{-1} \cdot \widetilde{\tau}$ $(\Lambda \in M)$ form a system lattice $\tau$-functions on $\widetilde{U}$.

We apply this proposition for constructing lattice $\tau$-functions from ORG $\tau$-functions discussed in this paper. Fixing a nonzero constant $\kappa \in \mathbb{C}^{*}$, let $D \subset V$ be a subset such that $D+Q\left(E_{8}\right) \kappa=D$, and take an ORG $\tau$-function $\tau=\tau(x)$ on $D$ with $\delta=\kappa$ in the sense of Definition [2.1. We then define the action of $T_{v} w \in T(V) \rtimes W\left(E_{8}\right)$ $\left(v \in V, w \in W\left(E_{8}\right)\right)$ on $V$ by

$$
T_{v} w \cdot x=w \cdot x+\kappa v \quad(x \in V) .
$$


We denote by $\pi: \mathfrak{h} \rightarrow V$ the orthogonal projection to $\stackrel{\circ}{\mathfrak{h}}=V$ in (9.8), and by $\pi_{\kappa}: \mathfrak{h}_{\kappa} \rightarrow V$ its restriction to $\mathfrak{h}_{\kappa}$. This projection $\pi_{\kappa}: \mathfrak{h}_{\kappa} \rightarrow V$ is equivariant with respect to the action of $T(V) \rtimes W\left(E_{8}\right)$ and compatible with the scalar product in the sense $\left(v \mid \pi_{\kappa}(h)\right)=(v \mid h)$, $(v \in V, h \in \mathfrak{h})$. Introducing a subset $U$ of $\mathfrak{h}_{\kappa}$ by

$$
U=\pi_{\kappa}^{-1}(D)=\left\{h \in \mathfrak{h}_{\kappa} \mid \pi_{\kappa}(h) \in D\right\} \subset \mathfrak{h}_{\kappa},
$$

we regard $\tau$ as a function on $U$ through the projection $\pi_{\kappa}: U \rightarrow D$. Note that the isomorphism

$$
\gamma_{\kappa}: V \times \mathbb{C} \stackrel{\sim}{\rightarrow} \mathfrak{h}_{\kappa}: \gamma_{\kappa}(x, \mu)=x-\left(\frac{1}{2 \kappa}(x \mid x)+\mu\right) c+\kappa d,
$$

induces the parametrization $\gamma_{\kappa}: D \times \mathbb{C} \stackrel{\sim}{\rightarrow} U$ of $U=\pi_{\kappa}^{-1}(D)$ with an invariant parameter $\mu \in \mathbb{C}$. Then obtain a $W\left(E_{8}\right)$-invariant function $\widetilde{\tau}$ on

$$
\widetilde{U}=\bigsqcup_{w \in W\left(E_{8}\right)}\{w\} \times w \cdot U \subset W\left(E_{8}\right) \times \mathfrak{h}_{\kappa}
$$

by setting

$$
\widetilde{\tau}(w, h)=\tau\left(\pi_{\kappa}\left(w^{-1} . h\right)\right)=\tau\left(w^{-1} \cdot x\right) \quad\left(w \in W\left(E_{8}\right), h \in w . U\right),
$$

where $x=\pi_{\kappa}(h) \in w . D$. By Proposition 9.2 , the family of functions $\widetilde{\tau}_{\Lambda}=T_{\Lambda-e_{9}}^{-1} \cdot \widetilde{\tau}$ gives a system of lattice $\tau$-functions on $\widetilde{U}$. If we take the classical part $\alpha=\pi_{0}\left(\Lambda-e_{9}\right) \in Q\left(E_{8}\right)$ of $\Lambda-e_{9}$, then we have $\widetilde{\tau}_{\Lambda}=T_{\alpha}^{-1} \cdot \widetilde{\tau}$, and hence

$$
\widetilde{\tau}_{\Lambda}(w, h)=\widetilde{\tau}\left(w, T_{\alpha} \cdot h\right)=\tau\left(w^{-1} \cdot \pi_{\kappa}\left(T_{\alpha} \cdot h\right)\right)=\tau\left(w^{-1} \cdot(x+\kappa \alpha)\right),
$$

for any $w \in W\left(E_{8}\right)$ and $h \in w \cdot U$, where $x=\pi_{\kappa}(h) \in w . D$.

Theorem 9.3 Let $\kappa \in \mathbb{C}^{*}$ be a generic constant. Suppose that a subset $D \subseteq V$ is stable by $W\left(E_{8}\right)$ and $D+Q\left(E_{8}\right) \kappa=D$. Let $\tau(x)$ be an $O R G \tau$-function on $D$ with $\delta=\kappa$. For each $\Lambda \in M$, define a function $\widetilde{\tau}_{\Lambda}$ on $\widetilde{U}$ of (9.52) by

$$
\widetilde{\tau}_{\Lambda}(w, h)=\tau\left(w^{-1} \cdot(x+\kappa \alpha)\right) \quad\left(w \in W\left(E_{8}\right), h \in w \cdot U\right)
$$

with $\alpha=\pi_{0}\left(\Lambda-e_{9}\right) \in Q\left(E_{8}\right)$ and $x=\pi_{\kappa}(h) \in w . D$. Then $\widetilde{\tau}_{\Lambda}(\Lambda \in M)$ form a system of lattice $\tau$-functions on $\widetilde{U}$.

For example, we consider the hypergeometric the ORG $\tau$-function $\tau(x)=\tau_{+-}(x)$ of Theorem 8.1 defined on

$$
\begin{aligned}
& D=D_{\phi,-\varpi}=\bigsqcup_{n \in \mathbb{Z}} H_{\phi,-\varpi+n \kappa}, \\
& H_{\phi,-\varpi+n \kappa}=\{x \in V \mid(\phi \mid x)=-\varpi+n \kappa\} \quad(n \in \mathbb{Z}),
\end{aligned}
$$

under the identification $\delta=\kappa$. The components $\tau^{(n)}=\left.\tau\right|_{H_{\phi,-\varpi+n \kappa}}(n \in \mathbb{Z})$ are then given by $\tau^{(n)}(x)=0(n<0)$ and

$$
\tau^{(n)}(x)=\Psi_{n}\left(p^{\frac{1}{2}} q^{\frac{1}{2}(1-n)} u ; p, q\right)=\Psi_{n}\left(q^{\frac{1}{2}} u^{-1} ; p, q\right) \quad(n=0,1,2, \ldots)
$$


in terms of the elliptic hypergeometric integral (6.49). Here, $p=e(\varpi), q=e(\kappa)$ and $u_{j}=e\left(x_{j}\right)$ denote the multiplicative variables corresponding to $x_{j}(j=0,1, \ldots, 7)$. In this case, the subset $U=\pi_{\kappa}^{-1}(D) \subset \mathfrak{h}_{\kappa}$ is specified as $U=\bigsqcup_{n \in \mathbb{Z}} U_{n}$, where

$$
U_{n}=\left\{h \in \mathfrak{h}_{\kappa} \mid(\phi \mid h)=-\varpi+n \kappa\right\}=\left\{h \in \mathfrak{h}_{\kappa} \mid\left(\alpha_{8} \mid h\right)=\varpi+(1-n) \kappa\right\} \quad(n \in \mathbb{Z}) .
$$

We regard the ORG $\tau$-function $\tau(x)$ as a function on $U$ through the coordinates

$$
x_{j}=\varepsilon_{j}-\frac{1}{2}\left(\varepsilon_{0}-\varepsilon_{9}\right)+\frac{1}{2} \kappa \quad(j=1, \ldots, 8), \quad x_{0}=-x_{8} .
$$

The corresponding lattice $\tau$-functions $\widetilde{\tau}_{\Lambda}(\Lambda \in M)$ on

$$
\widetilde{U}=\bigsqcup_{w \in W\left(E_{8}\right)}\{w\} \times w \cdot U
$$

are defined as

$$
\widetilde{\tau}_{\Lambda}(w, h)=\tau\left(w^{-1} \cdot(x+\kappa \alpha)\right) \quad\left(w \in W\left(E_{8}\right), h \in w \cdot U\right),
$$

where $\alpha=\pi_{0}\left(\Lambda-e_{9}\right) \in Q\left(E_{8}\right)$ and $x=\pi_{\kappa}(h)$. Since

$$
e_{j}-e_{9}=v_{0}+v_{j}-\phi+c \quad(j=1, \ldots, 8),
$$

the nine fundamental $\tau$-functions $\widetilde{\tau}_{e_{j}}(j=1, \ldots, 9)$ are specified as

$$
\begin{aligned}
& \widetilde{\tau}_{e_{j}}(w, h)=\tau\left(w^{-1} \cdot\left(x+\kappa\left(v_{0}+v_{j}-\phi\right)\right) \quad(j=1, \ldots, 7),\right. \\
& \widetilde{\tau}_{e_{8}}(w, h)=\tau\left(w^{-1} \cdot(x-\kappa \phi)\right), \quad \widetilde{\tau}_{e_{9}}(w, h)=\tau\left(w^{-1} \cdot x\right) .
\end{aligned}
$$

for $w \in W\left(E_{8}\right)$ and $h \in w \cdot U$.

\subsection{Remarks on the $\mathbb{P}^{1} \times \mathbb{P}^{1}$ picture}

The difference Painlevé equation of type $E_{8}$ can also be formulated in terms of the configuration of generic eight points in $\mathbb{P}^{1} \times \mathbb{P}^{1}$. In this case, following the formulation of [6] we use the Picard lattice

$$
L=\mathbb{Z}_{1} \oplus \mathbb{Z} \mathrm{h}_{2} \oplus \mathbb{Z} \mathrm{e}_{1} \oplus \mathbb{Z} \mathrm{e}_{2} \oplus \cdots \oplus \mathbb{Z} \mathrm{e}_{8}
$$

with the symmetric bilinear form $(\cdot \mid \cdot): L \times L \rightarrow \mathbb{Z}$ defined by

$$
\begin{aligned}
& \left(\mathrm{h}_{i} \mid \mathrm{h}_{i}\right)=0 \quad(i=1,2), \quad\left(\mathrm{h}_{1} \mid \mathrm{h}_{2}\right)=\left(\mathrm{h}_{2} \mid \mathrm{h}_{1}\right)=-1, \\
& \left(\mathrm{e}_{i} \mid \mathrm{e}_{j}\right)=\delta_{i j} \quad(i, j=1, \ldots, 8) .
\end{aligned}
$$

If we denote by $(f, g)$ the inhomogeneous coordinates of $\mathbb{P}^{1} \times \mathbb{P}^{1}, \mathrm{~h}_{1}$ and $\mathrm{h}_{2}$ represent the classes of lines $f=$ const. and $g=$ const. respectively, and $\mathrm{e}_{1}, \ldots, \mathrm{e}_{8}$ the classes of exceptional divisors corresponding to the generic eight points. This Picard lattice and 
its symmetric bilinear form are identified with those we have used in the $\mathbb{P}^{2}$ picture through the change of bases

$$
\mathrm{h}_{1}=e_{0}-e_{2}, \mathrm{~h}_{2}=e_{0}-e_{1}, \mathrm{e}_{1}=e_{0}-e_{1}-e_{2}, \mathrm{e}_{j}=e_{j+1}(j=2, \ldots, 8),
$$

and

$$
e_{0}=\mathrm{h}_{1}+\mathrm{h}_{2}-\mathrm{e}_{1}, e_{1}=\mathrm{h}_{1}-\mathrm{e}_{1}, e_{2}=\mathrm{h}_{2}-\mathrm{e}_{1}, e_{j}=\mathrm{e}_{j-1} \quad(j=3, \ldots, 9) .
$$

The simple roots $\alpha_{0}, \alpha_{1}, \ldots, \alpha_{8}$ are now expressed as

$$
\alpha_{0}=\mathrm{e}_{1}-\mathrm{e}_{2}, \alpha_{1}=\mathrm{h}_{1}-\mathrm{h}_{2}, \alpha_{2}=\mathrm{h}_{2}-\mathrm{e}_{1}-\mathrm{e}_{2}, \alpha_{j}=\mathrm{e}_{j-1}-\mathrm{e}_{j}(j=3, \ldots, 8) .
$$

The complex vector space $\mathfrak{h}=\mathbb{C} \otimes_{\mathbb{Z}} L$ is decomposed as $\mathfrak{h}=\grave{h} \oplus \mathbb{C} c \oplus \mathbb{C} d$ where

$$
c=2 \mathrm{~h}_{1}+2 \mathrm{~h}_{2}-\mathrm{e}_{1}-\cdots-\mathrm{e}_{8}, \quad d=-\mathrm{e}_{8}-\frac{1}{2} c .
$$

In this realization, the orthonormal basis $\left\{v_{0}, v_{1}, \ldots, v_{8}\right\}$ for $V=\stackrel{\circ}{\mathfrak{h}}$ is given by

$$
\begin{aligned}
& v_{1}=\mathrm{h}_{1}-\mathrm{e}_{1}-\frac{1}{2}\left(\mathrm{~h}_{1}+\mathrm{h}_{2}-\mathrm{e}_{1}-\mathrm{e}_{8}\right)+\frac{1}{2} c, \\
& v_{2}=\mathrm{h}_{2}-\mathrm{e}_{1}-\frac{1}{2}\left(\mathrm{~h}_{1}+\mathrm{h}_{2}-\mathrm{e}_{1}-\mathrm{e}_{8}\right)+\frac{1}{2} c, \\
& v_{j}=\mathrm{e}_{j-1}-\frac{1}{2}\left(\mathrm{~h}_{1}+\mathrm{h}_{2}-\mathrm{e}_{1}-\mathrm{e}_{8}\right)+\frac{1}{2} c(j=3, \ldots, 8), \quad v_{0}=-v_{8} .
\end{aligned}
$$

Accordingly, the coordinates $x=\left(x_{0}, x_{1}, \ldots, x_{7}\right)$ for $V$ are given by

$$
\begin{aligned}
& x_{1}=\eta_{1}-\epsilon_{1}-\frac{1}{2}\left(\eta_{1}+\eta_{2}-\epsilon_{1}-\epsilon_{8}\right)+\frac{1}{2} \delta, \\
& x_{2}=\eta_{2}-\epsilon_{1}-\frac{1}{2}\left(\eta_{1}+\eta_{2}-\epsilon_{1}-\epsilon_{8}\right)+\frac{1}{2} \delta, \\
& x_{j}=\epsilon_{j-1}-\frac{1}{2}\left(\eta_{1}+\eta_{2}-\epsilon_{1}-\epsilon_{8}\right)+\frac{1}{2} \delta(j=3, \ldots, 8), \quad x_{0}=-x_{8} .
\end{aligned}
$$

where $\eta_{i}=\left(\mathrm{h}_{i} \mid \cdot\right)(i=1,2)$ and $\epsilon_{j}=\left(\mathrm{e}_{j} \mid \cdot\right)(j=1, \ldots, 8)$.

In this framework, a system of lattice $\tau$-functions is defined as a family of dependent variables $\tau_{\Lambda}$, indexed by the same orbit

$$
M=W\left(E_{8}^{(1)}\right)\left\{\mathrm{e}_{1}, \ldots, \mathrm{e}_{8}\right\}=W\left(E_{8}^{(1)}\right) \mathrm{e}_{8} \subset L,
$$

which admit an action of $W\left(E_{8}^{(1)}\right)$ such that

$$
w \cdot \tau_{\Lambda}=\tau_{w \cdot \Lambda} \quad\left(w \in W\left(E_{8}^{(1)}\right), \Lambda \in M\right)
$$

and satisfy the quadratic relations

$$
\sigma_{\mathrm{e}_{j k}} \sigma_{\mathrm{e}_{r ; j k}} \tau_{\mathrm{e}_{i}} \tau_{\mathrm{h}_{r}-\mathrm{e}_{i}}+\sigma_{\mathrm{e}_{k i}} \sigma_{\mathrm{e}_{r ; k i}} \tau_{\mathrm{e}_{j}} \tau_{\mathrm{h}_{r}-\mathrm{e}_{j}}+\sigma_{\mathrm{e}_{i j}} \sigma_{\mathrm{e}_{r ; i j}} \tau_{\mathrm{e}_{k}} \tau_{\mathrm{h}_{r}-\mathrm{e}_{k}}=0
$$

for $r=1,2$ and for mutually distinct $i, j, k \in\{1, \ldots, 8\}$, where $\mathrm{e}_{i j}=\mathrm{e}_{i}-\mathrm{e}_{j}$ and $\mathrm{e}_{r ; i j}=\mathrm{h}_{r}-\mathrm{e}_{i}-\mathrm{e}_{j}$. Through the expression $\tau_{\Lambda}=T_{\Lambda-\mathrm{e}_{8}}^{-1} \tau_{\mathrm{e}_{8}}$, this notion of lattice $\tau$ functions is interpreted by that of ORG $\tau$-functions in the same way as in Proposition 9.2 and Theorem 9.3 . 


\section{Appendix}

\section{A Proof of Theorem 3.3}

In this Appendix, we give a proof of Theorem 3.3. The following proof is essentially the same as the argument in Masuda [8, Section 3]. We first prove Theorem 3.3 under an additional assumption that $\tau^{(n-1)}(x)$ and $\tau^{(n)}(x)$ are $W\left(E_{7}\right)$-invariant. After that we explain how the general case can be reduced to the invariant case.

\section{A.1 Preliminary remark}

We begin by a general remark on the Hirota equations associated with $C_{3}$-frames. Fixing a $C_{l}$-frame $A=\left\{ \pm a_{1}, \ldots, \pm a_{l}\right\}(l=3, \ldots, 8)$, we suppose that a function $\tau(x)$ satisfies the Hirota equation

$$
\tau\left(x \pm a_{i} \delta\right)\left[\left(a_{j} \pm a_{k} \mid x\right)\right]+\tau\left(x \pm a_{j} \delta\right)\left[\left(a_{k} \pm a_{i} \mid x\right)\right]+\tau\left(x \pm a_{k} \delta\right)\left[\left(a_{i} \pm a_{j} \mid x\right)\right]=0
$$

for any triple $i, j, k \in\{1, \ldots, l\}$. We also assume that $\left[\left(a_{i} \pm a_{j} \mid x\right)\right] \neq 0$ on the domain of definition of $\tau$ for any distinct pair $i, j \in\{1, \ldots, l\}$. Then (A.1) can be written as

$$
\tau\left(x \pm a_{k} \delta\right)=\frac{\tau\left(x \pm a_{i} \delta\right)\left[\left(a_{k} \pm a_{j} \mid x\right)\right]-\tau\left(x \pm a_{j} \delta\right)\left[\left(a_{k} \pm a_{i} \mid x\right)\right]}{\left[\left(a_{i} \pm a_{j} \mid x\right)\right]}
$$

for any $k \in\{1, \ldots, l\}$. In view of this expression, for each $u \in V$ we define

$$
f_{i j}(x ; u)=\frac{\tau\left(x \pm a_{i}\right)\left[\left(u \pm a_{j} \mid x\right)\right]-\tau\left(x \pm a_{j}\right)\left[\left(u \pm a_{i} \mid x\right)\right]}{\left[\left(a_{i} \pm a_{j} \mid x\right)\right]}
$$

for each distinct pair $i, j \in\{1, \ldots, l\}$, so that $f_{i j}\left(x ; a_{k}\right)=\tau\left(x \pm a_{k} \delta\right)(k \in\{1, \ldots, l\})$. A simple but important observation is that the three-term relation (2.1) of the function $[z]$ implies the functional equation

$$
f_{i j}\left(x ; u_{0}\right)\left[\left(u_{1} \pm u_{2} \mid x\right)\right]+f_{i j}\left(x ; u_{1}\right)\left[\left(u_{2} \pm u_{0} \mid x\right)\right]+f_{i j}\left(x ; u_{2}\right)\left[\left(u_{0} \pm u_{1} \mid x\right)\right]=0
$$

for any $u_{0}, u_{1}, u_{2} \in V$. From this, for any distinct pair $r, s \in\{1, \ldots, l\}$ we obtain

$$
\begin{aligned}
f_{i j}(x ; u)\left[\left(a_{r} \pm a_{s} \mid x\right)\right] & =f_{i j}\left(x ; a_{r}\right)\left[\left(u \pm a_{s} \mid x\right)\right]-f_{i j}\left(x ; a_{s}\right)\left[\left(u \pm a_{r} \mid x\right)\right] \\
& =\tau\left(x \pm a_{r} \delta\right)\left[\left(u \pm a_{s} \mid x\right)\right]-\tau\left(x \pm a_{s} \delta\right)\left[\left(u \pm a_{r} \mid x\right)\right] \\
& =f_{r s}(x ; u)\left[\left(a_{r} \pm a_{s} \mid x\right)\right],
\end{aligned}
$$

and hence $f_{i j}(x ; u)=f_{r s}(x ; u)$. Summarizing these arguments, we have

Lemma A.1 Let $A=\left\{ \pm a_{1}, \ldots, \pm a_{l}\right\}$ be a $C_{l}$-frame and suppose that $\tau(x)$ satisfies the Hirota equation (A.1) for any triple $i, j, k \in\{1, \ldots, l\}$. Then the function

$$
f(x ; u)=\frac{\tau\left(x \pm a_{i}\right)\left[\left(u \pm a_{j} \mid x\right)\right]-\tau\left(x \pm a_{j}\right)\left[\left(u \pm a_{i} \mid x\right)\right]}{\left[\left(a_{i} \pm a_{j} \mid x\right)\right]} \quad(x, u \in V)
$$


does not depend on the choice of distinct $i, j \in\{1, \ldots, l\}$. Furthermore, it satisfies

$$
f\left(x ; u_{0}\right)\left[\left(u_{1} \pm u_{2} \mid x\right)\right]+f\left(x ; u_{1}\right)\left[\left(u_{2} \pm u_{0} \mid x\right)\right]+f\left(x ; u_{2}\right)\left[\left(u_{0} \pm u_{1} \mid x\right)\right]=0
$$

for any $u_{0}, u_{1}, u_{2} \in V$, and

$$
f\left(x ; a_{k}\right)=\tau\left(x \pm a_{k} \delta\right) \quad(k=1, \ldots, l) .
$$

Returning to the setting of Theorem 3.3, we suppose furthermore that $\tau^{(n-1)}(x)$ on $H_{c+(n-1) \delta}$ and $\tau^{(n)}(x)$ on $H_{c+n \delta}$ are $W\left(E_{7}\right)$-invariant. For a function $\varphi=\varphi(x)$, the action $w . \varphi$ of $w \in W\left(E_{7}\right)$ is defined by $(w \cdot \varphi)(x)=\varphi\left(w^{-1} \cdot x\right)$. We say that $\varphi$ is invariant with respect to $w$ if $w \cdot \varphi=\varphi$, namely, $\varphi\left(w^{-1} . x\right)=\varphi(x)$. Note also that, for the function $\psi$ defined by $\psi(x)=\varphi(x+v)(v \in V)$, we have $(w \cdot \psi)(x)=(w \cdot \varphi)(x+w \cdot v)$, and hence $(w \cdot \psi)(x)=\varphi(x+w \cdot v)$ if $\varphi$ is $w$-invariant.

\section{A.2 Definition of $\tau^{(n+1)}: W\left(E_{7}\right)$-invariance and $\left(\mathrm{II}_{1}\right)_{n}$}

We first show that there exists a unique $W\left(E_{7}\right)$-invariant (meromorphic) function $\tau^{(n+1)}(x)$ on $H_{c+(n+1) \delta}$ that satisfies the bilinear equations of type $\left(\mathrm{II}_{1}\right)_{n}$.

We consider the $C_{8}$-frame $A=\left\{ \pm a_{0}, \pm a_{1}, \ldots, \pm a_{7}\right\}$ defined by

$$
\begin{aligned}
& a_{0}=\frac{1}{2}\left(v_{0}-v_{1}+\phi\right), \quad a_{1}=\frac{1}{2}\left(v_{1}-v_{0}+\phi\right), \\
& a_{j}=v_{j}+\frac{1}{2}\left(v_{0}+v_{1}-\phi\right) \quad(j=2, \ldots, 7) .
\end{aligned}
$$

Note that $\left(\phi \mid a_{0}\right)=\left(\phi \mid a_{1}\right)=1$ and $\left(\phi \mid a_{j}\right)=0(j=2, \ldots, 7)$. Then, from the assumption that $\tau^{(n)}(x)$ satisfies the bilinear equations of type $\left(\mathrm{II}_{0}\right)_{n}$, by Lemma A.1 it follows that the function

$$
f(x ; u)=\frac{\tau^{(n)}\left(x \pm a_{i} \delta\right)\left[\left(u \pm a_{j} \mid x\right)\right]-\tau^{(n)}\left(x \pm a_{j} \delta\right)\left[\left(u \pm a_{i} \mid x\right)\right]}{\left[a_{i} \pm a_{j}\right]}
$$

does not depend on the choice of distinct $i, j \in\{2, \ldots, 7\}$. In view of the bilinear equations of type $\left(\mathrm{II}_{1}\right)_{n}$, we define the function $\tau^{(n+1)}(x)$ on $H_{c+(n+1) \delta}$ by the equation

$$
\tau^{(n+1)}\left(x+a_{0} \delta\right) \tau^{(n-1)}\left(x-a_{0} \delta\right)=f\left(x ; a_{0}\right) .
$$

This implies that $\tau^{(n+1)}(x)$ satisfies

$$
\begin{aligned}
& \tau^{(n+1)}\left(x+a_{0} \delta\right) \tau^{(n-1)}\left(x-a_{0} \delta\right)\left[\left(a_{i} \pm a_{j} \mid x\right)\right] \\
& =\tau^{(n)}\left(x \pm a_{i} \delta\right)\left[\left(a_{0} \pm a_{j} \mid x\right)\right]-\tau^{(n)}\left(x \pm a_{j} \delta\right)\left[\left(a_{0} \pm a_{i} \mid x\right)\right]
\end{aligned}
$$

for any distinct $i, j \in\{2, \ldots, 7\}$. By replacing $x$ with $x-a_{0} \delta$, these equations can be written as

$$
\begin{aligned}
& \tau^{(n+1)}(x) \\
& =\frac{\tau^{(n)}\left(x-\left(a_{0} \pm a_{i}\right) \delta\right)\left[\left(a_{0} \pm a_{j} \mid x\right)-\delta\right]-\tau^{(n)}\left(x-\left(a_{0} \pm a_{j}\right) \delta\right)\left[\left(a_{0} \pm a_{i} \mid x\right)-\delta\right]}{\tau^{(n-1)}\left(x-2 a_{0} \delta\right)\left[\left(a_{i} \pm a_{j} \mid x\right)\right]}
\end{aligned}
$$


for any distinct $i, j \in\{2, \ldots, 7\}$. In terms of the basis $v_{0}, v_{1}, \ldots, v_{7}$ for $V$, we have

$$
\begin{aligned}
\tau^{(n+1)}(x)= & \frac{1}{\tau^{(n-1)}\left(x-\left(\phi+v_{0}-v_{1}\right) \delta\right)\left[\left(v_{j}-v_{i} \mid x\right)\right]\left[\left(\phi-v_{01 i j} \mid x\right)\right]} \\
& \cdot\left(\tau^{(n)}\left(x-v_{0 i} \delta\right) \tau^{(n)}\left(x-\left(\phi-v_{1 i}\right) \delta\right)\left[\left(v_{0 j} \mid x\right)-\delta\right]\left[\left(\phi-v_{1 j} \mid x\right)-\delta\right]\right. \\
& \left.\quad-\tau^{(n)}\left(x-v_{0 j} \delta\right) \tau^{(n)}\left(x-\left(\phi-v_{1 j}\right) \delta\right)\left[\left(v_{0 i} \mid x\right)-\delta\right]\left[\left(\phi-v_{1 i} \mid x\right)-\delta\right]\right)
\end{aligned}
$$

for any distinct $i, j \in\{2, \ldots, 7\}$, where $v_{a b}=v_{a}+v_{b}$ and $v_{a b c d}=v_{a}+v_{b}+v_{c}+v_{d}$.

We next show that this function $\tau^{(n+1)}(x)$ is invariant under the action of the Weyl group

$$
W\left(E_{7}\right)=\left\langle s_{0}, s_{1}, \ldots, s_{6}\right\rangle ; \quad s_{0}=r_{\phi-v_{0123}}, s_{j}=r_{v_{j}-v_{j+1}} \quad(j=1, \ldots, 6) .
$$

Since $\tau^{(n-1)}(x)$ and $\tau^{(n)}(x)$ are $W\left(E_{7}\right)$-invariant, from the fact that the expression (A.14) does not depend on the choice of $i, j \in\{2, \ldots, 7\}$, it follows that $\tau^{(n+1)}(x)$ is invariant with respect to $s_{2}, \ldots, s_{6}$. To see the invariance with respect to $s_{0}$, we take $(i, j)=(2,3)$ :

$$
\begin{aligned}
\tau^{(n+1)}(x)= & \frac{1}{\tau^{(n-1)}\left(x-\left(\phi+v_{0}-v_{1}\right) \delta\right)\left[\left(v_{3}-v_{2} \mid x\right)\right]\left[\left(\phi-v_{0123} \mid x\right)\right]} \\
& \cdot\left(\tau^{(n)}\left(x-v_{02} \delta\right) \tau^{(n)}\left(x-\left(\phi-v_{12}\right) \delta\right)\left[\left(v_{03} \mid x\right)-\delta\right]\left[\left(\phi-v_{13} \mid x\right)-\delta\right]\right. \\
& \left.-\tau^{(n)}\left(x-v_{03} \delta\right) \tau^{(n)}\left(x-\left(\phi-v_{13}\right) \delta\right)\left[\left(v_{02} \mid x\right)-\delta\right]\left[\left(\phi-v_{12} \mid x\right)-\delta\right]\right) .
\end{aligned}
$$

Since $s_{0}\left(v_{i j}\right)=\phi-v_{k l}$ for $\{i, j, k, l\}=\{0,1,2,3\}$, this expression is manifestly invariant with respect to $s_{0}$. It remains to show that $\tau^{(n+1)}(x)$ is invariant with respect to $s_{1}$. The $s_{1}$-invariance of $\tau^{(n+1)}(x)$ is equivalent to the equality of

$$
\begin{aligned}
L= & \tau^{(n-1)}\left(x-\left(\phi+v_{0}-v_{2}\right) \delta\right)\left[\left(v_{3}-v_{1} \mid x\right)\right] \\
& \cdot\left(\tau^{(n)}\left(x-v_{02} \delta\right) \tau^{(n)}\left(x-\left(\phi-v_{12}\right) \delta\right)\left[\left(v_{03} \mid x\right)-\delta\right]\left[\left(\phi-v_{13} \mid x\right)-\delta\right]\right. \\
& \left.\quad-\tau^{(n)}\left(x-v_{03} \delta\right) \tau^{(n)}\left(x-\left(\phi-v_{13}\right) \delta\right)\left[\left(v_{02} \mid x\right)-\delta\right]\left[\left(\phi-v_{12} \mid x\right)-\delta\right]\right)
\end{aligned}
$$

and

$$
\begin{aligned}
R= & \tau^{(n-1)}\left(x-\left(\phi+v_{0}-v_{1}\right) \delta\right)\left[\left(v_{3}-v_{2} \mid x\right)\right] \\
& \cdot\left(\tau^{(n)}\left(x-v_{01} \delta\right) \tau^{(n)}\left(x-\left(\phi-v_{12}\right) \delta\right)\left[\left(v_{03} \mid x\right)-\delta\right]\left[\left(\phi-v_{23} \mid x\right)-\delta\right]\right. \\
& \left.\quad-\tau^{(n)}\left(x-v_{03} \delta\right) \tau^{(n)}\left(x-\left(\phi-v_{23}\right) \delta\right)\left[\left(v_{01} \mid x\right)-\delta\right]\left[\left(\phi-v_{12} \mid x\right)-\delta\right]\right) .
\end{aligned}
$$

Expanding these as $L=L_{1}-L_{2}$ and $R=R_{1}-R_{2}$, we look at

$$
\begin{aligned}
& L_{1}-R_{1} \\
& =\tau^{(n)}\left(x-\left(\phi-v_{12}\right) \delta\right)\left[\left(v_{03} \mid x\right)-\delta\right] \\
& \quad \cdot\left(\tau^{(n-1)}\left(x-\left(\phi+v_{0}-v_{2}\right) \delta\right) \tau^{(n)}\left(x-v_{02} \delta\right)\left[\left(v_{3}-v_{1} \mid x\right)\right]\left[\left(\phi-v_{13} \mid x\right)-\delta\right]\right. \\
& \left.\quad-\tau^{(n-1)}\left(x-\left(\phi+v_{0}-v_{1}\right) \delta\right) \tau^{(n)}\left(x-v_{01} \delta\right)\left[\left(v_{3}-v_{2} \mid x\right)\right]\left[\left(\phi-v_{23} \mid x\right)-\delta\right]\right) .
\end{aligned}
$$


Setting $u_{i}=\frac{1}{2} \phi-v_{i}$ and $y=x-\left(\frac{1}{2} \phi+v_{0}\right)$, we compute the last factor as

$$
\begin{aligned}
& \tau^{(n-1)}\left(y-u_{2} \delta\right) \tau^{(n)}\left(y+u_{2} \delta\right)\left[\left(u_{1} \pm u_{3} \mid y\right)\right] \\
&-\tau^{(n-1)}\left(y-u_{1} \delta\right) \tau^{(n)}\left(y+u_{1} \delta\right)\left[\left(u_{2} \pm u_{3} \mid y\right)\right] \\
&= \tau^{(n-1)}\left(y-u_{3} \delta\right) \tau^{(n)}\left(y+u_{3} \delta\right)\left[\left(u_{1} \pm u_{2} \mid y\right)\right] \\
&= \tau^{(n-1)}\left(x-\left(\phi+v_{0}-v_{3}\right) \delta\right) \tau^{(n)}\left(x-v_{03} \delta\right)\left[\left(v_{2}-v_{1} \mid x\right)\right]\left[\left(\phi-v_{12} \mid x\right)-\delta\right]
\end{aligned}
$$

by the bilinear equation of type $(\mathrm{I})_{n-1 / 2}$ for the $C_{3}$-frame $\left\{ \pm u_{1}, \pm u_{2}, \pm u_{3}\right\}$. Hence we have

$$
\begin{aligned}
L_{1}-R_{1}= & \tau^{(n-1)}\left(x-\left(\phi+v_{0}-v_{3}\right) \delta\right) \tau^{(n)}\left(x-v_{03} \delta\right) \tau^{(n)}\left(x-\left(\phi-v_{12}\right) \delta\right) \\
& \cdot\left[\left(v_{03} \mid x\right)-\delta\right]\left[\left(v_{2}-v_{1} \mid x\right)\right]\left[\left(\phi-v_{12} \mid x\right)-\delta\right] .
\end{aligned}
$$

On the other hand, we have

$$
\begin{aligned}
& L_{2}-R_{2} \\
& =\tau^{(n)}\left(x-v_{03} \delta\right)\left[\left(\phi-v_{12} \mid x\right)-\delta\right] \\
& \quad \cdot\left(\tau^{(n-1)}\left(x-\left(\phi+v_{0}-v_{2}\right) \delta\right) \tau^{(n)}\left(x-\left(\phi-v_{13}\right)\right)\left[\left(v_{3}-v_{1} \mid x\right)\right]\left[\left(v_{02} \mid x\right)-\delta\right]\right. \\
& \left.\quad-\tau^{(n-1)}\left(x-\left(\phi+v_{0}-v_{1}\right) \delta\right) \tau^{(n)}\left(x-\left(\phi-v_{23}\right) \delta\right)\left[\left(v_{3}-v_{2} \mid x\right)\right]\left[\left(v_{01} \mid x\right)-\delta\right]\right) .
\end{aligned}
$$

Setting $b_{i}=\frac{1}{2} v_{0123}-v_{i}(i=0,1,2,3)$ and $z=x-\left(\phi-b_{0}\right) \delta$, we compute the last factor as

$$
\begin{aligned}
\tau^{(n-1)}\left(z-b_{2} \delta\right) \tau^{(n)}\left(z+b_{2} \delta\right)\left[\left(b_{1} \pm b_{3} \mid z\right)\right] \\
\quad-\tau^{(n-1)}\left(z-b_{1} \delta\right) \tau^{(n)}\left(z+b_{1} \delta\right)\left[\left(b_{2} \pm b_{3} \mid z\right)\right] \\
=\tau^{(n-1)}\left(z-b_{3} \delta\right) \tau^{(n)}\left(z+b_{3} \delta\right)\left[\left(b_{1} \pm b_{2} \mid z\right)\right] \\
=\tau^{(n-1)}\left(x-\left(\phi+v_{0}-v_{3}\right) \delta\right) \tau^{(n)}\left(x-\left(\phi-v_{12}\right) \delta\right)\left[\left(v_{2}-v_{1} \mid x\right)\right]\left[\left(v_{03} \mid x\right)-\delta\right]
\end{aligned}
$$

by the bilinear equation of type $(\mathrm{I})_{n-1 / 2}$ for the $C_{3}$-frame $\left\{ \pm b_{1}, \pm b_{2}, \pm b_{3}\right\}$. Hence

$$
\begin{aligned}
L_{2}-R_{2}= & \tau^{(n-1)}\left(x-\left(\phi+v_{0}-v_{3}\right) \delta\right) \tau^{(n)}\left(x-v_{03} \delta\right) \tau^{(n)}\left(x-\left(\phi-v_{12}\right) \delta\right) \\
& \cdot\left[\left(v_{03} \mid x\right)-\delta\right]\left[\left(v_{2}-v_{1} \mid x\right)\right]\left[\left(\phi-v_{12} \mid x\right)-\delta\right] \\
= & L_{1}-R_{1}
\end{aligned}
$$

which implies $L=R$ as desired.

Recall that $W\left(E_{7}\right)$ acts transitively on the set of all $C_{3}$-frames of type $\mathrm{II}_{1}$. Since $\tau^{(n+1)}(x)$ is $W\left(E_{7}\right)$-invariant and satisfies (A.12), it readily satisfies the bilinear equations of type $\left(\mathrm{II}_{1}\right)_{n}$ for all $C_{3}$-frames of type $\mathrm{II}_{1}$.

\section{A.3 $\left(\mathrm{II}_{1}\right)_{n} \Longrightarrow\left(\mathrm{II}_{2}\right)_{n}$}

Since $\tau^{(n+1)}(x)$ is $W\left(E_{7}\right)$-invariant, we have only to show that it satisfies the bilinear equation of type $\left(\mathrm{II}_{2}\right)_{n}$ for a particular $C_{3}$-frame of type $\mathrm{II}_{2}$. 
Taking the $C_{8}$-frame (A.9) of type II as before, we look at the bilinear relations

$$
\begin{aligned}
& \tau^{(n+1)}\left(x+a_{k} \delta\right) \tau^{(n-1)}\left(x-a_{k} \delta\right) \\
& =\frac{\tau^{(n)}\left(x \pm a_{2} \delta\right)\left[\left(a_{k} \pm a_{3} \mid x\right)\right]-\tau^{(n)}\left(x \pm a_{3} \delta\right)\left[\left(a_{k} \pm a_{2} \mid x\right)\right]}{\left[\left(a_{2} \pm a_{3} \mid x\right)\right]}
\end{aligned}
$$

of type $\left(\mathrm{II}_{1}\right)_{n}$ for $k=0,1$. From these we have

$$
\begin{aligned}
& \tau^{(n+1)}\left(x+a_{0} \delta\right) \tau^{(n-1)}\left(x-a_{0} \delta\right)\left[\left(a_{2} \pm a_{1} \mid x\right)\right] \\
&-\tau^{(n+1)}\left(x+a_{1} \delta\right) \tau^{(n-1)}\left(x-a_{1} \delta\right)\left[\left(a_{2} \pm a_{0} \mid x\right)\right] \\
&= \frac{\tau^{(n)}\left(x \pm a_{2} \delta\right)}{\left[\left(a_{2} \pm a_{3} \mid x\right)\right]}\left(\left[\left(a_{0} \pm a_{3} \mid x\right)\right]\left[\left(a_{2} \pm a_{1} \mid x\right)\right]-\left[\left(a_{1} \pm a_{3} \mid x\right)\right]\left[\left(a_{2} \pm a_{0} \mid x\right)\right]\right) \\
&= \tau^{(n)}\left(x \pm a_{2} \delta\right)\left[\left(a_{0} \pm a_{1} \mid x\right)\right],
\end{aligned}
$$

which is the bilinear equation of type $\left(\mathrm{II}_{2}\right)_{n}$ for the $C_{3}$-frame $\left\{ \pm a_{0}, \pm a_{1}, \pm a_{2}\right\}$ of type $\mathrm{II}_{2}$.

\section{A.4 $\left(\mathrm{II}_{1}\right)_{n} \Longrightarrow(\mathrm{I})_{n+1 / 2}$}

We have only to show that the bilinear equation $(\mathrm{I})_{n+1 / 2}$ holds for some particular $C_{3}$-frame of type I.

Taking the $C_{8}$-frame of (A.9), we look at the bilinear relation

$$
\begin{aligned}
& \tau^{(n+1)}(x) \tau^{(n-1)}\left(x-\left(\phi+v_{0}-v_{1}\right) \delta\right)\left[\left(v_{3}-v_{2} \mid x\right)\right]\left[\left(\phi-v_{0123} \mid x\right)\right] \\
& =\tau^{(n)}\left(x-\left(\phi-v_{12}\right) \delta\right) \tau^{(n)}\left(x-v_{02} \delta\right)\left[\left(v_{03} \mid x\right)-\delta\right]\left[\left(\phi-v_{13} \mid x\right)-\delta\right] \\
& \left.\quad-\tau^{(n)}\left(x-\left(\phi-v_{13}\right) \delta\right) \tau^{(n)}\left(x-v_{03}\right) \delta\right)\left[\left(v_{02} \mid x\right)-\delta\right]\left[\left(\phi-v_{12} \mid x\right)-\delta\right]
\end{aligned}
$$

of type $\left(\mathrm{II}_{1}\right)_{n}$. Replacing $x$ with $x+\left(v_{0}-v_{1}\right) \delta$, we rewrite this formula into

$$
\begin{aligned}
& \tau^{(n+1)}\left(x+\left(v_{0}-v_{1}\right) \delta\right) \tau^{(n-1)}(x-\phi \delta)\left[\left(v_{3}-v_{2} \mid x\right)\right]\left[\left(\phi-v_{0123} \mid x\right)\right] \\
& =\tau^{(n)}\left(x-\left(\phi-v_{02}\right) \delta\right) \tau^{(n)}\left(x-v_{12} \delta\right)\left[\left(v_{03} \mid x\right)\right]\left[\left(\phi-v_{13} \mid x\right)\right] \\
& \quad-\tau^{(n)}\left(x-\left(\phi-v_{03}\right) \delta\right) \tau^{(n)}\left(x-\left(v_{13}\right) \delta\right)\left[\left(v_{02} \mid x\right)\right]\left[\left(\phi-v_{12} \mid x\right)\right] .
\end{aligned}
$$

Multiplying this by $\tau^{(n)}\left(x+\left(v_{01}-\phi\right) \delta\right)\left[\left(\phi-v_{23} \mid x\right)\right]$, we obtain

$$
\begin{aligned}
& \tau^{(n+1)}\left(x+\left(v_{0}-v_{1}\right) \delta\right) \tau^{(n)}\left(x+\left(v_{01}-\phi\right) \delta\right)\left[\left(v_{3}-v_{2} \mid x\right)\right]\left[\left(\phi-v_{23} \mid x\right)\right] \\
& \cdot \tau^{(n-1)}(x-\phi \delta)\left[\left(\phi-v_{0123} \mid x\right)\right] \\
&= \tau^{(n)}\left(x-u_{01} \delta\right) \tau^{(n)}\left(x-u_{02} \delta\right) \tau^{(n)}\left(x-v_{12} \delta\right)\left[\left(u_{13} \mid x\right)\right]\left[\left(u_{23} \mid x\right)\right]\left[\left(v_{03} \mid x\right)\right] \\
&-\tau^{(n)}\left(x-u_{01} \delta\right) \tau^{(n)}\left(x-u_{03} \delta\right) \tau^{(n)}\left(x-v_{13} \delta\right)\left[\left(u_{12} \mid x\right)\right]\left[\left(u_{23} \mid x\right)\right]\left[\left(v_{02} \mid x\right)\right]
\end{aligned}
$$

where $u_{i}=\frac{1}{2} \phi-v_{i}$ and $u_{i j}=u_{i}+u_{j}=\phi-v_{i j}$. Then, applying the cyclic permutation (123) to this formula, we see that the sum of those three vanishes term by term. It means that

$$
\tau^{(n+1)}\left(x+\left(v_{0}-v_{1}\right) \delta\right) \tau^{(n)}\left(x+\left(v_{01}-\phi\right) \delta\right)\left[\left(v_{3}-v_{2} \mid x\right)\right]\left[\left(\phi-v_{23} \mid x\right)\right]+\cdots=0,
$$


namely

$$
\tau^{(n+1)}\left(x+\left(u_{1}-u_{0}\right) \delta\right) \tau^{(n)}\left(x-\left(u_{0}+u_{1}\right) \delta\right)\left[\left(u_{2} \pm u_{3} \mid x\right)\right]+\cdots=0 .
$$

Replacing $x$ by $x+u_{0} \delta$, we obtain the bilinear equation

$$
\tau^{(n+1)}\left(x+u_{1} \delta\right) \tau^{(n)}\left(x-u_{1} \delta\right)\left[\left(u_{2} \pm u_{3} \mid x\right)\right]+\cdots=0
$$

of type $(\mathrm{I})_{n+1 / 2}$ for the $C_{3}$-frame $\left\{ \pm u_{1}, \pm u_{2}, \pm u_{3}\right\}$ of type I.

\section{A.5 $\left(\mathrm{II}_{1}\right)_{n} \Longrightarrow\left(\mathrm{II}_{0}\right)_{n+1}$}

In order to show that $\tau^{(n+1)}(x)$ satisfies the bilinear equations of type (II $)_{n+1}$, we take the $C_{8}$-frame $A=\left\{ \pm a_{0}, \pm a_{1}, \ldots \pm a_{7}\right\}$ of type II defined by

$$
\begin{aligned}
& a_{j}=v_{j}+\frac{1}{2}\left(v_{67}-\phi\right) \quad(j=0,1,2,3,4,5) ; \\
& a_{6}=\frac{1}{2}\left(v_{6}-v_{7}+\phi\right), \quad a_{7}=\frac{1}{2}\left(v_{7}-v_{6}+\phi\right) .
\end{aligned}
$$

We prove that $\tau^{(n+1)}(x)$ satisfies the bilinear equation

$$
\tau^{(n+1)}\left(x+\left(a_{0} \pm a_{3}\right) \delta\right)\left[\left(a_{4} \pm a_{5} \mid x\right)\right]+\cdots=0
$$

of type $\left(\mathrm{II}_{0}\right)_{n+1}$ for the $C_{3}$-frame $\left\{ \pm a_{3}, \pm a_{4}, \pm a_{5}\right\}$. In terms of the basis $v_{0}, v_{1}, \ldots, v_{7}$ for $V$, this equation is expressed as

$$
\tau^{(n+1)}\left(x+\left(v_{0}-v_{3}\right) \delta\right) \tau^{(n+1)}\left(x+\left(\phi-v_{1245}\right) \delta\right)\left[\left(v_{4}-v_{5} \mid x\right)\right]\left[\left(\phi-v_{0123} \mid x\right)\right]+\cdots=0 .
$$

We now look at the bilinear equation

$$
\begin{gathered}
\tau^{(n+1)}\left(x+\left(v_{0}-v_{4}\right) \delta\right) \tau^{(n-1)}(x-\phi \delta)\left[\left(v_{2}-v_{1} \mid x\right)\right]\left[\left(\phi-v_{0124} \mid x\right)\right] \\
=\tau^{(n)}\left(x-\left(\phi-v_{01}\right) \delta\right) \tau^{(n)}\left(x-v_{14} \delta\right)\left[\left(v_{02} \mid x\right)\right]\left[\left(\phi-v_{24} \mid x\right)\right] \\
-\tau^{(n)}\left(x-\left(\phi-v_{02}\right) \delta\right) \tau^{(n)}\left(x-v_{24} \delta\right)\left[\left(v_{01} \mid x\right)\right]\left[\left(\phi-v_{14} \mid x\right)\right]
\end{gathered}
$$

of type $\left(\mathrm{II}_{1}\right)_{n}$. Applying $s_{0}$ to this formula, we obtain

$$
\begin{aligned}
& \tau^{(n+1)}\left(x+\left(\phi-v_{1234}\right) \delta\right) \tau^{(n-1)}(x-\phi \delta)\left[\left(v_{2}-v_{1} \mid x\right)\right]\left[\left(v_{3}-v_{4} \mid x\right)\right] \\
& =\tau^{(n)}\left(x-v_{23} \delta\right) \tau^{(n)}\left(x-v_{14} \delta\right)\left[\left(\phi-v_{13} \mid x\right)\right]\left[\left(\phi-v_{24} \mid x\right)\right] \\
& \left.\quad-\tau^{(n)}\left(x-v_{13}\right) \delta\right) \tau^{(n)}\left(x-v_{24} \delta\right)\left[\left(\phi-v_{23} \mid x\right)\right]\left[\left(\phi-v_{14} \mid x\right)\right] .
\end{aligned}
$$


Using these formulas, we compute

$$
\begin{aligned}
& \tau^{(n+1)}\left(x+\left(v_{0}-v_{3}\right) \delta\right) \tau^{(n+1)}\left(x+\left(\phi-v_{1245}\right) \delta\right)\left[\left(v_{4}-v_{5} \mid x\right)\right]\left[\left(\phi-v_{0123} \mid x\right)\right] \\
& \cdot \tau^{(n-1)}(x-\phi)^{2}\left[\left(v_{2}-v_{1} \mid x\right)\right]^{2} \\
&=\left(\tau^{(n)}\left(x-u_{01} \delta\right) \tau^{(n)}\left(x-v_{13} \delta\right)\left[\left(v_{02} \mid x\right)\right]\left[\left(u_{23} \mid x\right)\right]\right. \\
&\left.\left.\quad-\tau^{(n)}\left(x-u_{02}\right) \delta\right) \tau^{(n)}\left(x-v_{23} \delta\right)\left[\left(v_{01} \mid x\right)\right]\left[\left(u_{13} \mid x\right)\right]\right) \\
& \cdot\left(\tau^{(n)}\left(x-v_{24} \delta\right) \tau^{(n)}\left(x-v_{15} \delta\right)\left[\left(u_{14} \mid x\right)\right]\left[\left(u_{25} \mid x\right)\right]\right. \\
&\left.\left.\quad-\tau^{(n)}\left(x-v_{14}\right) \delta\right) \tau^{(n)}\left(x-v_{25} \delta\right)\left[\left(u_{24} \mid x\right)\right]\left[\left(u_{15} \mid x\right)\right]\right) \\
&= \tau^{(n)}\left(x-u_{01} \delta\right)\left[\left(v_{02} \mid x\right)\right] \\
& \cdot\left\{\tau^{(n)}\left(x-v_{13} \delta\right) \tau^{(n)}\left(x-v_{24}\right) \delta\right) \tau^{(n)}\left(x-v_{15} \delta\right)\left[\left(u_{23} \mid x\right)\right]\left[\left(u_{14} \mid x\right)\right]\left[\left(u_{25} \mid x\right)\right] \\
&\left.\left.\quad-\tau^{(n)}\left(x-v_{13} \delta\right) \tau^{(n)}\left(x-v_{14}\right) \delta\right) \tau^{(n)}\left(x-v_{25} \delta\right)\left[\left(u_{23} \mid x\right)\right]\left[\left(u_{24} \mid x\right)\right]\left[\left(u_{15} \mid x\right)\right]\right\} \\
&+\tau^{(n)}\left(x-u_{02} \delta\right)\left[\left(v_{01} \mid x\right)\right] \\
& \cdot\left\{\tau^{(n)}\left(x-v_{23} \delta\right) \tau^{(n)}\left(x-v_{14}\right) \delta\right) \tau^{(n)}\left(x-v_{25} \delta\right)\left[\left(u_{13} \mid x\right)\right]\left[\left(u_{24} \mid x\right)\right]\left[\left(u_{15} \mid x\right)\right] \\
&\left.\left.\quad-\tau^{(n)}\left(x-v_{23} \delta\right) \tau^{(n)}\left(x-v_{24}\right) \delta\right) \tau^{(n)}\left(x-v_{15} \delta\right)\left[\left(u_{13} \mid x\right)\right]\left[\left(u_{14} \mid x\right)\right]\left[\left(u_{25} \mid x\right)\right]\right\}
\end{aligned}
$$

Applying the cyclic permutation (345) to this formula, we can directly observe that the sum of those three vanishes term by term. This completes the proof of Theorem $3.3 \mathrm{in}$ the case where $\tau^{(n-1)}(x)$ and $\tau^{(n)}(x)$ are $W\left(E_{7}\right)$-invariant.

\section{A.6 General case}

We now consider the general case where $\tau^{(n-1)}(x)$ and $\tau^{(n)}(x)$ are not necessarily $W\left(E_{7}\right)$ invariant. In such a situation, we need to deal with all the transforms $w \cdot \tau^{(n-1)}$ and $w \cdot \tau^{(n)}$ by $w \in W\left(E_{7}\right)$ simultaneously.

For each hyperplane $H_{\kappa}=\{x \in V \mid(\phi \mid x)=\kappa\}(\kappa \in \mathbb{C})$ perpendicular to $\phi$, we introduce the covering space

$$
\widetilde{H}_{\kappa}=W\left(E_{7}\right) \times H_{\kappa},
$$

and define the action of $w \in W\left(E_{7}\right)$ and the translation $T_{v}\left(v \in H_{0}\right)$ by

$$
w \cdot(g, x)=(w g, w \cdot x), \quad T_{v} \cdot(g, x)=(g, x+v \delta) \quad\left(g \in W\left(E_{7}\right), x \in H_{\kappa}\right),
$$

so that $w T_{v}=T_{w . v} w$. For each function $\psi$ on $\widetilde{H}_{\kappa}$, the induced actions $w . \psi\left(w \in W\left(E_{7}\right)\right)$ and $T_{v} \cdot \psi\left(v \in H_{0}\right)$ are described as

$$
(w \cdot \psi)(g, x)=\psi\left(w^{-1} g, w^{-1} x\right), \quad\left(T_{v} \cdot \psi\right)(g, x)=\psi(g, x-v \delta) .
$$

For each function $\varphi$ on $H_{\kappa}$, we define a function $\widetilde{\varphi}$ on $\widetilde{H}_{\kappa}$ by

$$
\widetilde{\varphi}(g, x)=(g \cdot \varphi)(x)=\varphi\left(g^{-1} \cdot x\right) \quad\left(g \in W\left(E_{7}\right), x \in H_{\kappa}\right) .
$$

Then this function $\widetilde{\varphi}$ is $W\left(E_{7}\right)$-invariant, and $\varphi$ is recovered from $\widetilde{\varphi}$ by $\varphi(x)=\widetilde{\varphi}(1, x)$ $\left(x \in H_{\kappa}\right)$. Conversely, a function $\psi$ on $\widetilde{H}_{\kappa}$ is $W\left(E_{7}\right)$-invariant if and only if it is obtained as the lift $\psi=\widetilde{\varphi}$ of a function $\varphi$ on $H_{\kappa}$. 
Returning to the setting of Theorem 3.3, for the functions $\tau^{(k)}$ on $H_{c+k \delta}$ we consider the lifts $\widetilde{\tau}^{(k)}$ on $\widetilde{H}_{c+k \delta}(k=n-1, n)$. Note that, for each $g \in W\left(E_{7}\right), g \cdot \tau^{(n-1)}$ and $g \cdot \tau^{(n)}$ also satisfy the bilinear equations $(\mathrm{I})_{n-1 / 2}$ and $\left(\mathrm{II}_{0}\right)_{n}$. Hence, the lifted functions $\widetilde{\tau}^{(k)}(k=n-1, n)$ satisfy the bilinear equations corresponding to $(\mathrm{I})_{n-1 / 2}$ and $\left(\mathrm{II}_{0}\right)_{n}$. Formally, those bilinear equations can be written as

$$
(\mathrm{I})_{n-1 / 2}: \quad\left[\left(a_{1} \pm a_{2} \mid x\right)\right] \widetilde{\tau}^{(n-1)}\left(g, x-a_{0}\right) \widetilde{\tau}^{(n)}\left(g, x+a_{0}\right)+\cdots=0
$$

and

$$
(\mathrm{II})_{n}: \quad\left[\left(a_{1} \pm a_{2} \mid x\right)\right] \widetilde{\tau}^{(n)}\left(g, x \pm a_{0}\right)+\cdots=0 .
$$

As in (A.12), we can define a function $\psi=\psi(g, x)$ on $\widetilde{H}_{c+(n+1) \delta}$ so that

$$
\begin{aligned}
& \psi\left(g, x+a_{0} \delta\right) \widetilde{\tau}^{(n-1)}\left(g, x-a_{0} \delta\right)\left[\left(a_{i} \pm a_{j} \mid x\right)\right] \\
& =\widetilde{\tau}^{(n)}\left(g, x-\left(a_{0} \pm a_{i}\right) \delta\right)\left[\left(a_{0} \pm a_{j} \mid x\right)\right]-\widetilde{\tau}^{(n)}\left(g, x \pm a_{j} \delta\right)\left[\left(a_{0} \pm a_{i} \mid x\right)\right]
\end{aligned}
$$

for any distinct $i, j \in\{2, \ldots, 7\}$. Since $\widetilde{\tau}^{(k)}$ are $W\left(E_{7}\right)$-invariant on $\widetilde{H}_{c+k \delta}(k=n-1, n)$, applying the previous arguments to the lifted functions, we see that $\psi$ is also $W\left(E_{7}\right)$ invariant and satisfies the bilinear equations corresponding to $\left(\mathrm{II}_{1}\right)_{n},\left(\mathrm{II}_{2}\right)_{n},(\mathrm{I})_{n+1 / 2}$, $\left(\mathrm{II}_{0}\right)_{n+1}$. Since $\psi$ is $W\left(E_{7}\right)$-invariant, it is expressed as $\psi=\widetilde{\tau}^{(n+1)}$ with a function on $\tau^{(n+1)}$ on $H_{c+(n+1) \delta}$ :

$$
\psi(g, x)=\widetilde{\tau}^{(n+1)}(g, x)=\left(g \cdot \tau^{(n+1)}\right)(x), \quad \tau^{(n+1)}(x)=\psi(1, x) .
$$

Then the bilinear equations $\left(\mathrm{II}_{1}\right)_{n},\left(\mathrm{II}_{2}\right)_{n},(\mathrm{I})_{n+1 / 2},\left(\mathrm{II}_{0}\right)_{n+1}$ for $\widetilde{\tau}^{(n-1)}, \widetilde{\tau}^{(n)}$ and $\psi=$ $\widetilde{\tau}^{(n+1)}$ means that $\tau^{(n+1)}$ satisfies the corresponding bilinear equations of four types and that the recursive construction of $\tau^{(n+1)}$ from $\tau^{(n-1)}, \tau^{(n)}$ is equivariant with respect to the action of $W\left(E_{7}\right)$.

\section{Acknowledgments}

The author would like to thank his coworkers, Kenji Kajiwara, Tetsu Masuda, Yasuhiro Ohta and Yasuhiko Yamada, who allowed him to write in this form a paper largely based on the collaboration with them. He is also grateful to Yasushi Komori and Junichi Shiraishi for providing valuable comments and suggestions in various stages of this work.

\section{Funding}

This work was supported by JSPS Kakenhi Grant (B)15H03626. 


\section{References}

[1] J.H. Conway and N.J.A. Sloane: Sphere Packings, Lattice and Groups, Second Edition, Springer Verlag, 1993.

[2] I. Dolgachev and D. Ortland: Point Sets in Projective Spaces and Theta Functions, Astérisque No. 165 (1988), 210 pp. (1989).

[3] V. Kac: Infinite Dimensional Lie Algebras, Third Edition, Cambridge University Press, 1990.

[4] K. Kajiwara, T. Masuda, M. Noumi, Y. Ohta and Y. Yamada: ${ }_{10} E_{9}$ solution to the elliptic Painlevé equation, J. Phys. A 36 (2003), L263-L272.

[5] K. Kajiwara, T. Masuda, M. Noumi, Y. Ohta and Y. Yamada: Point configurations, Cremona transformations and the elliptic difference Painlevé equation, Théories asymptotiques et équations de Painlevé (Angers, juin 2004), Séminaires et Congrès 14 (2006), 169-198.

[6] K. Kajiwara, M. Noumi and Y. Yamada: Geometric aspects of Painlevé equations, to appear in J. Phys. A, Topical Review. (arXiv1509.08168, 167 pages)

[7] Y. Komori: Elliptic Ruijsenaars operators and elliptic hypergeometric integrals, Elliptic Integrable Systems (M. Noumi and K. Takasaki, Eds.), Rokko Lectures in Mathematics 18 (2005), 49-56.

[8] T. Masuda: Hypergeometric $\tau$-functions of the $q$-Painlevé system of type $E_{8}^{(1)}$, Ramanujan J. 24 (2011), 1-31.

[9] M. Noumi: Padé interpolation and hypergeometric series, Algebraic and Analytic Aspects of Integrable Systems and Painlevé Equations (A. Dzhamay, K. Maruno and C.M. Ormerod, Eds.), Contemp. Math. 651 (2015), 1-23. (arXiv1503.02147, 25 pages)

[10] M. Noumi, S. Tsujimoto and Y. Yamada: Padé interpolation for elliptic Painlevé equation, Symmetries, Integrable Systems and Representations (K. Iohara, S. Morier-Genoud and B. Rémy, Eds.), pp. 463-482, Springer Proceedings in Mathematics and Statistics 40, Springer 2013.

[11] Y. Ohta, A. Ramani and B. Grammaticos: An affine Weyl group approach to the eight-parameter discrete Painlevé equation, Symmetries and Integrability of Difference Equations (Tokyo, 2000), J. Phys. A 34 (2001), 10523-10532.

[12] E.M. Rains: Symmetries and recurrences of elliptic hypergeometric integrals, Elliptic Integrable Systems (M. Noumi and K. Takasaki, Eds.), Rokko Lectures in Mathematics 18 (2005), 183-199.

[13] E.M. Rains: Transformations of elliptic hypergeometric integrals, Ann. of Math. 171 (2010), 169-243. 
[14] E.M. Rains: An isomonodromy interpretation of the hypergeometric solutions of the elliptic Painlevé equation (and generalizations), SIGMA 7 (2011) Paper 088, $24 \mathrm{pp}$

[15] H. Sakai: Rational surfaces associated with affine root systems and geometry of the Painlevé equations, Comm. Math. Phys. 220 (2001), 165-229.

[16] V.P. Spiridonov: Theta hypergeometric integrals, Algebra i Analiz 15(2003), 161215, St. Petersburg Math. J. 15 (2004), 929-967.

[17] V.P. Spiridonov: Classical elliptic hypergeometric functions and their applications, Elliptic Integrable Systems (M. Noumi and K. Takasaki, Eds.), Rokko Lectures in Mathematics 18 (2005), 253-287.

[18] S. O. Warnaar: Summation and transformation formulas for elliptic hypergeometric series, Constr. Approx. 18 (2002), 479-502.

[19] E.T. Whittaker and G.N. Watson: A Course of Modern Analysis, 4th Edition, Cambridge University Press, 1927. 